\title{
Homotopy theory of $G$-diagrams and equivariant excision
}

\author{
EMANUELE DotTo \\ KRISTIAN MOI
}

\begin{abstract}
Let $G$ be a finite group. We define a suitable model-categorical framework for $G$-equivariant homotopy theory, which we call $G$-model categories. We show that the diagrams in a $G$-model category which are equipped with a certain equivariant structure admit a model structure. This model category of equivariant diagrams supports a well-behaved theory of equivariant homotopy limits and colimits. We then apply this theory to study equivariant excision of homotopy functors.
\end{abstract}

55N91, 55P91; 55P65, 55P42

\section{Introduction}

The concept of $G$-diagram was introduced, under different names, in Villarroel-Flores's thesis [29] and independently by Jackowski and Słomińska [16], and they were further studied by Villarroel-Flores [30]. In the current literature the theory of $G$-diagrams has been only partially developed. It is limited, due to the fact that it is used for very specific applications, to properties of homotopy colimits of $G$-diagrams in the category of spaces or of simplicial sets (see eg [16] or Thévenaz and Webb [27]). The contribution of the present paper is a systematic treatment of $G$-diagrams in a nice (simplicial, cofibrantly generated etc) model category. An immediate advantage of this general theory is that it allows us to work in the category of genuine $G$-spectra. Additionally, it is the first treatment of homotopy limits of $G$-diagrams. As an application of this abstract framework, we set up a theory of equivariant enriched homotopy functors and formulate an "equivariant excision" condition in terms of cubical $G$-diagrams. This condition agrees with Goodwillie's notion of excision [13] when $G$ is the trivial group, and with Blumberg's definition [4] for the category of $G$-spaces.

Given a finite group $G$ acting on a category $I$ by functors $a(g): I \rightarrow I$, a $G-$ diagram in a category $\mathscr{C}$ is a functor $X: I \rightarrow \mathscr{C}$ together with natural transformations $g_{X}: X \rightarrow X \circ a(g)$ for every $g$ in $G$, which are compatible with the group structure. A map of $G$-diagrams is a natural transformation between the underlying diagrams that commutes with the structure maps (see Definitions 1.1 and 1.2). We write $\mathscr{C}_{a}^{I}$ for the resulting category of $G$-diagrams. The category $\mathscr{C}_{a}^{I}$ is isomorphic to the category 
of diagrams in $\mathscr{C}$ indexed on the Grothendieck construction of the action functor $a: G \rightarrow$ Cat (see Lemma 1.9 and [16, Section 2]). If the category of $G$-objects $\mathscr{C}^{G}$ is a sufficiently nice model category, such as $G$-spaces with the fixed point model structure, or orthogonal $G$-spectra with the genuine $G$-stable model structure, we prove:

Theorem 2.6 Let $\mathscr{C}$ be a $G$-model category (see Definition 2.1). There is a cofibrantly generated $\mathrm{sSet}^{G}$-enriched model structure on the category of $G$-diagrams $\mathscr{C}_{a}^{I}$ with weak equivalences (resp fibrations) the maps of $G$-diagrams $f: X \rightarrow Y$ such that the value $f_{i}$ at the object $i \in$ ob $I$ is a weak equivalence (resp fibration) in the model category $\mathscr{C}^{G_{i}}$ of objects with an action of the stabilizer group $G_{i}$.

The authors first became interested in $G$-diagrams while working on equivariant delooping results for so-called real algebraic $K$-theory and real topological Hochschild homology. A recurring example of a $G$-diagram in this work is the following:

Example Let $X$ be a pointed space with an action of $C_{2}$, the cyclic group of order two, with $\sigma: X \rightarrow X$ representing the action of the non-trivial group element. A diagram of pointed spaces

$$
Y \stackrel{p}{\longrightarrow} X \stackrel{q}{\longleftarrow} Z
$$

together with mutually inverse homeomorphisms $r: Y \rightarrow Z$ and $l: Z \rightarrow Y$ which cover $\sigma$, in the sense that $p \circ l=\sigma \circ q$ and $q \circ r=\sigma \circ p$, defines a $C_{2}$-diagram of pointed spaces. The pullback $Y \times_{X} Z$ inherits a natural $C_{2}$-action given by $(y, z) \mapsto(l(z), r(y))$, and similarly the homotopy pullback

$$
Y \times{ }_{X}^{h} Z=\left\{(y, \gamma, z) \in Y \times X^{I} \times Z \mid p(y)=\gamma(0) \text { and } \gamma(1)=q(z)\right\}
$$

inherits the action $(y, \gamma, z) \mapsto(l(z), \sigma \circ \bar{\gamma}, r(y))$, where $\bar{\gamma}(t)=\gamma(1-t)$. The usual inclusion $Y \times_{X} Z \hookrightarrow Y \times_{X}^{h} Z$ is equivariant with respect to these actions. Let $\mathbb{R}^{1,1}$ denote the sign representation of $C_{2}$ on $\mathbb{R}$ and let $\Omega^{1,1} X$ be the space of pointed maps from the one point compactification $S^{\mathbb{R}^{1,1}}$ to $X$ with $C_{2}$ acting by conjugation. If $Y$ (and hence $Z$ ) is contractible, then a contracting homotopy induces a $C_{2}$-homotopy equivalence

$$
Y \times_{X}^{h} Z \simeq \Omega^{1,1} X .
$$

On underlying spaces, this just an instance of the well-known homotopy equivalence

$$
\Omega X \simeq \operatorname{holim}(* \rightarrow X \leftarrow *) \text {. }
$$

This example illustrates how limits and homotopy limits of punctured $C_{2}$-squares of spaces carry a $C_{2}$-action and how these can be used to construct the loop space 
by the sign representation of $C_{2}$. More generally, when it makes sense to talk about the limit, colimit, homotopy limit or homotopy colimit of a $G$-diagram $X$ in any ambient category $\mathscr{C}$, these constructions have natural $G$-actions induced by the structure maps $g_{X}$ (see Corollary 1.5 and Section 1.2). Moreover, the usual comparison maps $\lim X \rightarrow \operatorname{holim} X$ and hocolim $X \rightarrow \operatorname{colim} X$ are equivariant, as we already observed for the $C_{2}$-diagram (1). In general, most constructions involving (co)limits and (co)ends enrichments applied to $G$-diagrams produce $G$-objects and equivariant maps between them. The homotopy limits and colimits of $G$-diagrams are homotopy invariant in the following sense:

Corollary 2.22 The functors holim: $\mathscr{C}_{a}^{I} \rightarrow \mathscr{C}^{G}$ and hocolim: $\mathscr{C}_{a}^{I} \rightarrow \mathscr{C}^{G}$ preserve equivalences between fibrant diagrams and point-wise cofibrant diagrams, respectively.

We prove other fundamental properties of these equivariant homotopy limits and colimits functors, analogous to classical theorems from homotopy theory of diagrams:

- Theorem 2.25 A homotopy cofinality theorem for homotopy limits and colimits of $G$-diagrams, generalizing the results [27, Theorem 1; 30, Section 6].

- Corollary 2.26 A twisted Fubini theorem, showing that homotopy colimits of $G$-diagrams over a Grothendieck construction can be calculated "point-wise" (an equivariant analogue of Chachólski and Scherer [6, Proposition 26.5]). As an immediate corollary we obtain an equivariant analogue of Thomason's homotopy colimit theorem [28].

- Theorem 2.28 An Elmendorf theorem, showing that for suitable ambient categories one can equivalently define the homotopy theory of $G$-diagrams by replacing $G$ with the opposite of its orbit category (a diagrammatic analogue of the classical result of [11]).

As an application of this model categorical theory of $G$-diagrams, we define and study equivariant excision. Classically, a homotopy invariant functor between model categories is excisive if it sends homotopy cocartesian squares to homotopy cartesian squares (see [13]). Blumberg [4] shows that this notion is not well behaved when the categories involved are categories of $G$-objects; enriched homotopy functors on the category of pointed $G$-spaces $\operatorname{Top}_{*}^{G} \rightarrow \operatorname{Top}_{*}^{G}$ that are classically linear (excisive and sending the point to a $G$-contractible space) are a model only for the category of naïve $G$-spectra. In order to model genuine $G$-spectra, one needs a property stronger than classical linearity. Blumberg achieves this by adding an extra condition to linearity, a compatibility condition with equivariant Spanier-Whitehead duality. 
Here we take a different approach to equivariant excision, following the idea that the relation between equivariant excision and excision should resemble the relation between genuine $G$-spectra and naïve $G$-spectra. Instead of adding an extra condition to classical excision, we replace squares by "equivariant cubes", similarly to the way one replaces integers with $G$-representations in defining $G$-spectra. For a finite $G$ set $J$ we consider the poset category $\mathcal{P}(J)$ of subsets of $J$ ordered by inclusion. This category inherits a $G$-action from the $G$-action on $J$.

Definition ( $G$-excision) A $J$-cube $X$ in $\mathscr{C}$ is a $G$-diagram in $\mathscr{C}$ shaped over $\mathcal{P}(J)$, ie it is an object of $\mathscr{C}_{a}^{\mathcal{P}(J)}$. We say that $X$ is homotopy cartesian if the canonical map

$$
X_{\varnothing} \longrightarrow \operatorname{holim}_{\mathcal{P}(J) \backslash \varnothing} X
$$

is a weak equivalence in the model category of $G$-objects $\mathscr{C}^{G}$. Dually, it is homotopy cocartesian if the canonical map hocolim $\operatorname{P}_{\mathcal{P}(J) \backslash J} X \rightarrow X_{J}$ is an equivalence in $\mathscr{C}^{G}$. A suitably homotopy invariant functor $\Phi: \mathscr{C}^{G} \rightarrow \mathscr{D}^{G}$ is called $G$-excisive if it sends homotopy cocartesian $G_{+}-$cubes to homotopy cartesian $G_{+}-$cubes.

Here $G_{+}$is the set $G$ with an added disjoint basepoint on which $G$ acts by left multiplication. It plays the role of a "regular" $G$-set, analogous to the regular representation of $G$ in stable equivariant homotopy theory. The added basepoint has an important role, discussed in detail in Remark 3.15. We prove in Proposition 3.31 that this notion of $G$-excision is equivalent to Blumberg's definition [4] when $\mathscr{C}$ is the category of pointed spaces. The paper contains a series of fundamental properties of $G$-excision, which appropriately reflect the fundamental properties of excision to a genuine equivariant context. They can be summarized as follows:

- Remark 3.14 A $G$-excisive functor $\mathscr{C}^{G} \rightarrow \mathscr{D}^{G}$ is classically excisive, that is, it sends homotopy cocartesian squares in $\mathscr{C}^{G}$ to homotopy cartesian squares in $\mathscr{D}^{G}$.

- Corollary 3.23 A $G$-linear functor is also $H$-linear for every subgroup $H$ of $G$.

- Proposition 3.36 Every enriched $G$-linear homotopy functor $\Phi$ from finite $G-\mathrm{CW}$-complexes to $G$-spectra is equivalent to one of the form $E_{\Phi} \wedge(-)$ for some $G$-spectrum $E_{\Phi}$.

- Theorem 3.35 The identity functor on $G$-spectra is $G$-excisive: for any finite $G$-set $J$, a $J$-cube of spectra is homotopy cartesian if and only if it is homotopy cocartesian. 
- Theorem 3.20 Any $G$-excisive reduced homotopy functor $\Phi: \mathscr{C}^{G} \rightarrow \mathscr{D}^{G}$ satisfies the Wirthmüller isomorphism theorem, that is, the canonical map $\Phi\left(G \otimes_{H} c\right) \rightarrow \operatorname{hom}_{H}(G, \Phi(c))$ is an equivalence in $\mathscr{D}^{G}$ for every subgroup $H$ of $G$ and $H$-object $c$ of $\mathscr{C}^{H}$.

- Corollary 3.28 and Remark 3.29 If $\mathscr{D}^{G}$ is suitably presentable, a construction similar to Goodwillie's differential [13] defines a universal $G$-excisive approximation to any reduced homotopy functor $\mathscr{C}^{G} \rightarrow \mathscr{D}^{G}$.

These properties have interesting consequences for the identity functor on $G$-spectra. The fact that it is $G$-excisive shows that the theory of equivariant cubes provides a good context in which the category of $G$-spectra is " $G$-stable". Moreover, Theorem 3.20 applied to the identity functor on $G$-spectra gives a new proof of the classical Wirthmüller isomorphism theorem. An analysis of the structure of the proofs of Theorems 3.20 and 3.35 gives the following argument: The identity on $G$-spectra is $G$-excisive as a direct consequence of the equivariant Freudenthal suspension theorem, by formally manipulating homotopy limits and colimits. Given an $H$-equivariant spectrum $E$, there is an explicit homotopy cocartesian $(G / H)_{+}-$cube of spectra $W E$ with initial vertex $(W E)_{\varnothing}=G_{+} \wedge_{H} E$ and $\operatorname{holim}_{\mathcal{P}\left(G / H_{+}\right) \backslash \varnothing} W E=F_{H}\left(G_{+}, E\right)$. By $G-$ excision for the identity functor, $W E$ is homotopy cartesian, that is, the canonical map $G_{+} \wedge_{H} E \rightarrow F_{H}\left(G_{+}, E\right)$ is a stable equivalence of $G$-spectra.

Acknowledgments We wish to thank Irakli Patchkoria for help with proofreading and for pointing out a mistake in an earlier version of this article. We would also like to thank Ib Madsen for his enduring support and for encouraging us to write this paper.

Dotto was partially supported by the ERC Adv. Grant number 228082. Moi was supported by the Danish National Research Foundation through the Centre for Symmetry and Deformation (DNRF92).

\section{Definitions and setup}

\subsection{Categories of $G$-diagrams}

We first introduce some notation and conventions. If $\mathscr{C}$ is a (possibly large) category and $I$ is a small category, we write $\mathscr{C}^{I}$ for the usual category of functors from $I$ to $\mathscr{C}$. By topological space we will mean a compactly generated weak Hausdorff space and Top is the category of such spaces with continuous maps between them. We write $\operatorname{Map}(X, Y)$ for the space of maps from $X$ to $Y$ endowed with the compact-open topology. The based variants of the above are $\operatorname{Top}_{*}$ and $\operatorname{Map}_{*}(X, Y)$. 
In the following, $\mathscr{C}$ will be a category, $G$ a finite group and $I$ a small category. By a slight abuse of notations we will also write $G$ for the category with one object $*$ and one morphism $g: * \rightarrow *$ for each element $g \in G$, with composition given by $g \circ h=g h$. The group $G$ will act on $I$ from the left and we will encode the action as a functor $a: G \rightarrow$ Cat sending $*$ to $I$. Most of the content of this section can be found in the work of Jackowski and Słomińska [16] or Villarroel-Flores [30].

Definition 1.1 (cf [16, Definition 2.2; 30, Definition 3.1) Let $X: I \rightarrow \mathscr{C}$ be an $I-$ shaped diagram in $\mathscr{C}$. A $G$-structure on $X$ with respect to the action $a$ is a collection of natural transformations $\left\{g_{X}: X \rightarrow X \circ a(g)\right\}_{g \in G}$ such that

(1) $e_{X}=\mathrm{id}_{X}$,

(2) $\left(g_{X}\right)_{a(h)} \circ h_{X}=(g h)_{X}$ for all $g, h \in G$,

where $\left(g_{X}\right)_{a(h)}$ is the natural transformation obtained by restricting $g_{X}$ along the functor $a(h): I \rightarrow I$. An $I$-shaped diagram $X$ with a $G$-structure will be called an $I$-shaped $G$-diagram in $\mathscr{C}$ with respect to the action $a$, or simply a $G$-diagram in $\mathscr{C}$ if $I$ and $a$ are understood.

In order to simplify the notation we will mostly write $g$ instead of $a(g)$ when this does not cause confusion. Accordingly, when $X$ and $Y$ are $I$-indexed $G$-diagrams we will write $f_{g}$ for the restriction of a map $f: X \rightarrow Y$ along the functor $g=a(g): I \rightarrow I$. In the later sections we will sometimes write $g$ instead of $g_{X}$.

Definition 1.2 A map of $G$-diagrams $f: X \rightarrow Y$ is a natural transformation $f: X \rightarrow Y$ of underlying diagrams such that for each $g \in G$ the diagram

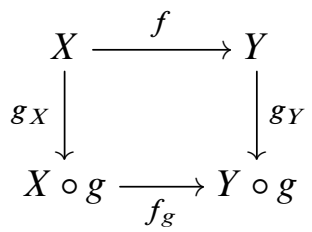

commutes in $\mathscr{C}^{I}$.

The composite of two maps of $G$-diagrams is again a map of $G$-diagrams. For a fixed action $a$ of the group $G$ on $I$ we write $\mathscr{C}_{a}^{I}$ for the category whose objects are the $G$-diagrams in $\mathscr{C}$ with respect to $a$ and with morphisms the maps of $G$-diagrams.

Example 1.3 Let $[n]$ be the usual category with objects $0,1, \ldots, n$ and a morphism $i \rightarrow j$ if and only if $i \leq j$. For a small category $I$ the nerve $N I$ is the usual simplicial 
set with $N I_{n}=\operatorname{Fun}([n], I)$. Taking over-categories gives a functor $N(I /-): I \rightarrow$ sSet. The $G$-action on $I$ gives maps $N_{/ i, g}: N(I / i) \rightarrow N(I / g i)$ for $g \in G$ and $i$ an object of $I$, by mapping

$$
\left(i_{0} \rightarrow \cdots \rightarrow i_{n} \rightarrow i\right) \stackrel{g}{\longmapsto}\left(g i_{0} \rightarrow \cdots \rightarrow g i_{n} \rightarrow g i\right) .
$$

These maps combine to give a $G$-diagram structure on $N(I /-)$. Similarly, the functor $N(-/ I)^{\text {op }}: I^{\text {op }} \rightarrow$ sSet with the maps $N_{i, g /}: N(i / I)^{\text {op }} \rightarrow N(g i / I)^{\text {op }}$ defines a $G-$ diagram in sSet.

Let $I$ and $J$ be small categories with $G$-actions $a$ and $b$, respectively, and let $F: I \rightarrow J$ be a functor. We say that $F$ is $G$-equivariant if it commutes strictly with the $G$-actions, that is, if $F(g i)=g F(i)$ and $F(g \alpha)=g F(\alpha)$ for all objects $i$ in $I$ and morphisms $\alpha$ in $I$. If $Y$ is a $J$-shaped $G$-diagram then the restriction $F^{*} Y=Y \circ F$ has a naturally induced $G$-structure with maps $g_{\left(F^{*} Y\right)}=F^{*}\left(g_{Y}\right)$.

Now assume that $\mathscr{C}$ is complete and cocomplete. Then the functor $F^{*}: \mathscr{C}^{J} \rightarrow \mathscr{C}^{I}$ has a left adjoint $F_{\text {! }}$ and a right adjoint $F_{*}$ given by left and right Kan extension, respectively. We will now see that, if $X$ is an $I$-shaped $G$-diagram, then there are natural $G$-structures on $F_{!} X$ and $F_{*} X$. We treat the left Kan extension first.

The value of the functor $F_{!} X$ on an object $j$ of $J$ is given by the coequalizer

$$
\coprod_{\left(i_{0} \stackrel{\alpha}{\rightarrow} i_{1}, f: F\left(i_{1}\right) \rightarrow j\right)} X_{i_{0}} \stackrel{s}{\longrightarrow} \coprod_{\left(i_{0}, f: F\left(i_{0}\right) \rightarrow j\right)} X_{i_{0}} \longrightarrow F_{!} X_{j},
$$

where $s$ projects onto the source of the indexing map $\alpha$ and $t$ maps into the target of $\alpha$ by the map $X(\alpha)$. For an element $g \in G$ the natural transformation $g_{X}$ induces a map of diagrams

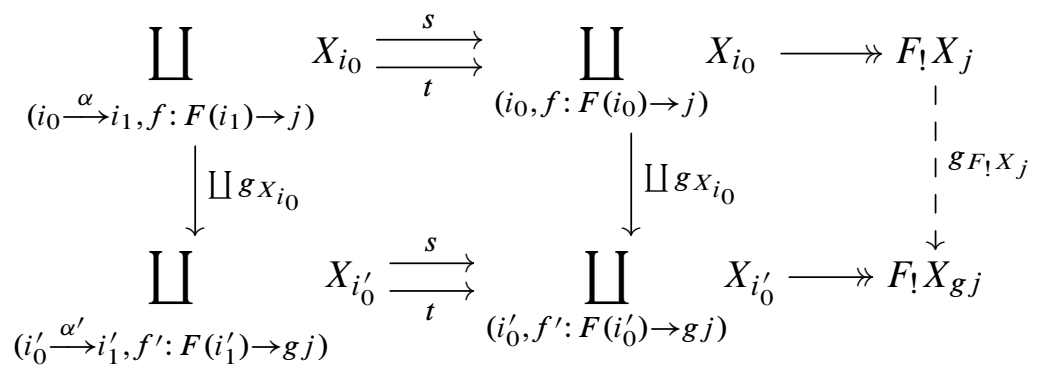

and the dotted arrow is the $j$-component of the natural transformation

$$
g_{F ! X}: F_{!} X \rightarrow\left(F_{!} X\right) \circ g .
$$


It is not hard to see that the set $\left\{g_{F_{!} X}\right\}_{g \in G}$ constitutes a $G$-structure on $F ! X$ and that the underlying functor $F_{!}$takes maps of $I$-indexed $G$-diagrams to maps of $J-$ indexed $G$-diagrams. Similarly, for the right Kan extension $F_{*}$ a dual construction with equalizers gives a $G$-structure $\left\{g_{F_{*} X}\right\}_{g \in G}$ on $F_{*} X$. We write simply $F_{!} X$ and $F_{*} X$ for the $G$-diagrams obtained in this way.

Proposition 1.4 The constructions $F_{!} X$ and $F_{*} X$ define functors $F_{!}, F_{*}: \mathscr{C}_{a}^{I} \rightarrow \mathscr{C}_{b}^{J}$.

A particularly interesting case of the above is when $J=*$ is the category with one object, one morphism and trivial $G$-action. In this case the functors $F_{!}$and $F_{*}$ are more commonly known as colim $I$ and $\lim _{I}$, respectively.

Corollary 1.5 Let $X$ be an $I$-indexed $G$-diagram. Then the above constructions induce natural left $G$-actions on $\operatorname{colim}_{I} X$ and $\lim _{I} X$.

Example 1.6 (Products and coproducts) Let $I$ be a discrete category with $G$-action, ie a $G$-set, and consider a $G$-diagram $X$ in the category Set of sets. The coproduct $\bigsqcup_{I} X$ is the set of pairs $(i, x)$ with $x \in X_{i}$ and the action of $g \in G$ is given by

$$
g(x, i)=\left(g_{X_{i}}(x), g i\right) .
$$

The product $\prod_{I} X$ is the set of functions $\boldsymbol{x}: I \rightarrow \bigcup_{i \in I} X_{i}$ such that $\boldsymbol{x}(i) \in X_{i}$ for all $i \in I$. The action of $g \in G$ on $x \in \prod_{I} X$ is determined by the equation

$$
(g \boldsymbol{x})(g i)=g_{X_{i}}(\boldsymbol{x}(i)) .
$$

This example generalizes to arbitrary categories with products and coproducts but the notation becomes more cumbersome when one can no longer speak about elements of objects.

We now give an alternative description of $G$-diagrams which is sometimes easier to work with.

Definition 1.7 Let $G \rtimes_{a} I$ be the following category:

- $\mathrm{ob} G \rtimes_{a} I=\mathrm{ob} I$.

- A morphism $i \rightarrow j$ in $G \rtimes_{a} I$ is a pair $(g, \alpha: g i \rightarrow j)$, where $g \in G$.

- Composition is given by $(h, \beta: h j \rightarrow k) \circ(g, \alpha: g i \rightarrow j)=(g h, \beta \circ h \alpha: g h i \rightarrow k)$.

Remark 1.8 The category $G \rtimes_{a} I$ is the Grothendieck construction of the functor $a: G \rightarrow$ Cat, sometimes denoted $G \int a$ (see eg [28]). 
A $G$-diagram $X$ gives rise to a functor $X^{\rtimes_{a}}: G \rtimes_{a} I \rightarrow \mathscr{C}$ by setting $X_{i}^{\rtimes_{a}}=X_{i}$ on objects and defining

$$
X^{\rtimes_{a}}(g, \alpha: g i \rightarrow j)=X(\alpha) \circ g_{X_{i}}
$$

on morphisms. We leave it to the reader to check that this respects composition of maps.

Lemma 1.9 The assignment $X \mapsto X^{\rtimes_{a}}$ is functorial and defines an isomorphism of categories

$$
\Phi: \mathscr{C}_{a}^{I} \stackrel{\sim}{\longrightarrow} \mathscr{C}^{G \rtimes_{a} I}
$$

Proof The functoriality is clear. We define a functor $\Phi^{\prime}: \mathscr{C}^{G \rtimes_{a} I} \rightarrow \mathscr{C}_{a}^{I}$ which is inverse to $\Phi$. For a diagram $Y: G \rtimes_{a} I \rightarrow \mathscr{C}$, define the underlying diagram of $\Phi^{\prime}(Y)$ to be $\left(\left.Y\right|_{I}\right)$, ie the restriction of $Y$ along the canonical inclusion $\iota: I \hookrightarrow G \rtimes_{a} I$ given by $\iota(i)=i$ and $\iota(\alpha: i \rightarrow j)=(e, \alpha: i \rightarrow j)$. For an element $g \in G$ the natural transformation $g_{\Phi^{\prime}(Y)}$ is defined at an object $i$ by $Y(g$, id: $g i \rightarrow g i)$. Both naturality of the $g_{\Phi^{\prime}(Y)}$ and conditions (1)-(2) from Definition 1.1 follow from the functoriality of $Y$ with respect to morphisms in $G \rtimes_{a} I$. For a natural transformation $f: Y \rightarrow Z$ in $\mathscr{C}^{G \rtimes_{a} I}$ we define $\Phi^{\prime}(f)=\left.f\right|_{I}$. It is now easy to check that the functors $\Phi$ and $\Phi^{\prime}$ are mutually inverse.

Corollary 1.10 Let $\mathscr{C}$ be a bicomplete category. Then $\mathscr{C}_{a}^{I}$ is also bicomplete.

Proof The diagram category $\mathscr{C}^{G \rtimes_{a} I}$ is bicomplete since $\mathscr{C}$ is. It follows from Lemma 1.9 that $\mathscr{C}_{a}^{I}$ is bicomplete.

\subsection{Enrichments and homotopy (co)limits}

If $\mathscr{C}$ is any category, then the category $\mathscr{C}^{G}$ is naturally enriched in left $G$-sets in the following way. For objects $c$ and $d$ of $\mathscr{C}^{G}$ let $\mathscr{C}(c, d)$ be the set of maps between the underlying objects in $\mathscr{C}$. Then $G$ acts on $\mathscr{C}(c, d)$ by conjugation:

$$
g \cdot f=g_{d} \circ f \circ\left(g^{-1}\right)_{c},
$$

where $\left(g^{-1}\right)_{c}$ and $g_{d}$ represent the actions of $g^{-1}$ and $g$ on $c$ and $d$, respectively. The fixed points set $\mathscr{C}(c, d)^{G}$ is precisely the set of $G$-equivariant maps from $c$ to $d$.

If $I$ is a small category with an action $a$ of $G$, then the category $\mathscr{C}_{a}^{I}$ of $G$-diagrams becomes enriched in left $G$-sets by taking $\mathscr{C}_{a}^{I}(X, Y)$ to be the set $\mathscr{C}^{I}(X, Y)$ of maps of underlying diagrams $f: X \rightarrow Y$, with action given by

$$
g \cdot f=\left(g_{Y}\right)_{g^{-1}} \circ f_{g^{-1}} \circ\left(g^{-1}\right)_{X} .
$$


If $f$ is fixed under the action of $G$, then

$$
f=g^{-1} f=\left(\left(g^{-1}\right)_{Y}\right)_{g} \circ f_{g} \circ g_{X}=\left(g_{Y}\right)^{-1} \circ f_{g} \circ g_{X} .
$$

In other words, $f$ is fixed if and only if the square

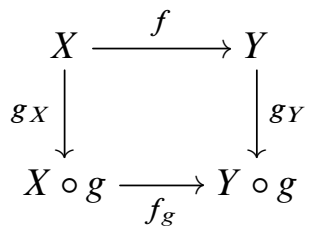

commutes for all $g \in G$. It follows that the fixed points $\underline{\mathscr{C}}_{a}^{I}(X, Y)^{G}$ are precisely the maps of $G$-diagrams $\mathscr{C}_{a}^{I}(X, Y)$. If $I=*$ then this statement reduces to the one above about maps in $\mathscr{C}^{G}$.

Proposition 1.11 Let $I$ and $J$ be small categories with $G$-actions $a$ and $b$, respectively. Let $F: I \rightarrow J$ be an equivariant functor. For an $I$-indexed $G$-diagram $X$ and a $J$-indexed $G$-diagram $Y$, the bijections

$$
\begin{aligned}
& \phi_{X, Y}: \underline{\mathscr{C}}_{a}^{I}\left(X, F^{*} Y\right) \simeq \underline{\mathscr{C}}_{b}^{J}\left(F_{!} X, Y\right), \\
& \psi_{X, Y}: \underline{\mathscr{C}}_{a}^{I}\left(F^{*} Y, X\right) \stackrel{\simeq}{\longrightarrow} \underline{\mathscr{C}}_{b}^{J}\left(Y, F_{*} X\right)
\end{aligned}
$$

induced by the adjunctions on underlying diagrams are $G$-equivariant.

Proof We show that $\phi=\phi_{X, Y}$ is equivariant; the argument for $\psi_{X, Y}$ is similar.

Let $f: X \rightarrow F^{*} Y$ be a map of diagrams and $g \in G$. Then $\phi(g \cdot f)$ is the unique map $F ! X \rightarrow Y$ such that the diagram

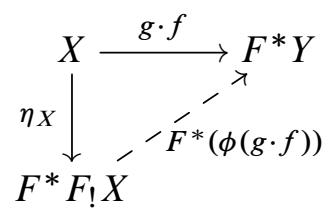

commutes, where $\eta_{X}$ is the unit of the $\left(F_{!}, F^{*}\right)$-adjunction at the object $X$. Consider the following diagram:

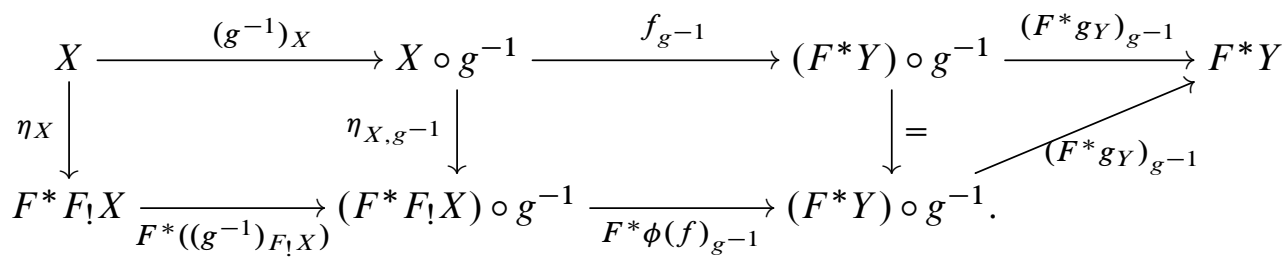


The commutativity of the left-hand square follows immediately from the definition of $g_{F_{!} X}$ and the middle square commutes by the definition of $\phi(f)$. Composing the maps in the top row gives

$$
\left(F^{*} g_{Y}\right)_{g^{-1}} \circ f_{g^{-1}} \circ\left(g^{-1}\right)_{X}=g \cdot f
$$

and composing along the bottom row from $F^{*} F_{!} X$ to $F^{*} Y$ gives

$$
F^{*}\left(\left(g_{Y}\right)_{g^{-1}} \circ \phi(f)_{g^{-1}} \circ\left(g^{-1}\right)_{F_{!} X}\right)=F^{*}(g \cdot \phi(f)) .
$$

It follows that $F^{*}(g \cdot \phi(f))$ defines a lift in the diagram (2) so, by uniqueness of the lift, we conclude that $\phi(g \cdot f)=g \cdot \phi(f)$.

Taking fixed points in Proposition 1.11 we immediately get the following:

Corollary 1.12 The functors $F_{!}$and $F_{*}$ are left and right adjoint, respectively, to the restriction functor $F^{*}: \mathscr{C}_{b}^{J} \rightarrow \mathscr{C}_{a}^{I}$. In particular, the diagonal $\Delta_{I}=p^{*}: \mathscr{C}^{G} \rightarrow \mathscr{C}_{a}^{I}$ induced by the projection $p: I \rightarrow *$ has left adjoint $p_{!}=\operatorname{colim}_{I}$ and right adjoint $p_{*}=\lim _{I}$.

Let $I$ be a category with $G$-action $a$ and let $G$ act diagonally on the product $I^{\text {op }} \times I$. Given a $G$-diagram $Z: I^{\mathrm{op}} \times I \rightarrow \mathscr{C}$ recall that the end $\int_{i} Z_{i, i}$ of $Z$ is the equalizer

$$
\int_{i} Z_{i, i} \longmapsto \prod_{i} Z_{i, i} \stackrel{s}{\longrightarrow} \prod_{\alpha: i \rightarrow j} Z_{j, i},
$$

where $s$ acts on the left and $t$ acts on the right by the map $\alpha$. The end $\int_{i} Z_{i, i}$ inherits a left $G$-action by the following maps:

$$
\begin{gathered}
\int_{i} Z_{i, i} \longrightarrow \prod_{i} Z_{i, i} \stackrel{s}{\longrightarrow} \prod_{\alpha: i \rightarrow j} Z_{j, i} \\
g_{\left(\int Z\right)} \\
\downarrow \prod_{i} g_{Z_{(i, i)}} \prod_{\alpha} g_{Z_{(j, i)}} \\
\int_{i}^{\downarrow} Z_{i, i} \longrightarrow \prod_{i} Z_{i, i} \underset{t}{\stackrel{s}{\longrightarrow}} \prod_{\alpha: i \rightarrow j}^{\downarrow} Z_{j, i}
\end{gathered}
$$

The coend $\int^{i} Z_{i, i}$ is the coequalizer

$$
\coprod_{\alpha: i \rightarrow j} Z_{j, i} \stackrel{s}{\longrightarrow} \coprod_{i} Z_{i, i} \longrightarrow \int_{i} Z_{i, i},
$$

which inherits a $G$-action in a similar way. 
Example 1.13 For $I$-diagrams $X$ and $Y$ in $\mathscr{C}$ we can describe the set of maps (natural transformations) between them as the end

$$
\mathscr{C}^{I}(X, Y)=\int_{i} \mathscr{C}\left(X_{i}, Y_{i}\right)
$$

Similarly, for $G$-diagrams $X$ and $Y$ in $\mathscr{C}_{a}^{I}$ there is a natural isomorphism of $G$-sets

$$
\underline{\mathscr{C}}_{a}^{I}(X, Y) \cong \int_{i} \mathscr{C}\left(X_{i}, Y_{i}\right)
$$

with the $G$-action on the left-hand term described above.

By a simplicial category we will mean a category $\mathscr{C}$ that is enriched, tensored and cotensored in simplicial sets, in the sense of eg Dugger and Shipley [10, Section 2.2] or Goerss and Jardine [12, Definition II.2.1]. This means that for any two objects $c$ and $d$ in $\mathscr{C}$ there is a simplicial set $\operatorname{Map}_{\mathscr{C}}(c, d)$ and a natural bijection $\mathscr{C}(c, d) \cong \operatorname{Map}_{\mathscr{C}}(c, d)_{0}$. Moreover, given a simplicial set $K$ there are objects $K \otimes c$ and $\operatorname{map}_{\mathscr{C}}(K, c)$ of $\mathscr{C}$. These satisfy some associativity constraints and naturality conditions, making $\operatorname{Map}_{\mathscr{C}}(-,-)$ and $\operatorname{map}_{\mathscr{C}}(-,-)$ contravariant functors in the first variable and covariant in the second variable and $-\otimes-$ covariant in both variables. Finally, for all $c$ and $d$ in $\mathscr{C}$ and $K$ in sSet there are natural isomorphisms in sSet,

$$
\operatorname{Map}_{\mathscr{C}}(K \otimes c, d) \cong \operatorname{Map}\left(K, \operatorname{Map}_{\mathscr{C}}(c, d)\right) \cong \operatorname{Map}_{\mathscr{C}}\left(c, \operatorname{map}_{\mathscr{C}}(K, d)\right),
$$

where Map with no subscript denotes the usual internal hom-object in sSet.

Using this structure we will now describe additional structure on the category $\mathscr{C}_{a}^{I}$ of $I$-indexed $G$-diagrams in a simplicial category $\mathscr{C}$. We begin with the enrichment. We noted above that for a pair $X, Y$ of $G$-diagrams in $\mathscr{C}$ the set $\mathscr{C}^{I}(X, Y)$ has a $G$-action induced by the $G$-structures on $X$ and $Y$. This gives $\mathscr{C}_{a}^{I}$ the structure of a category enriched in left $G$-sets. The functor $(i, j) \mapsto \operatorname{Map}_{\mathscr{C}}\left(X_{i}, Y_{j}\right)$ going from $I^{\mathrm{op}} \times I$ to sSet becomes a $G$-diagram by letting $g \in G$ act at $(i, j)$ by

$$
\operatorname{Map}_{\mathscr{C}}\left(g_{X_{i}}^{-1}, g_{Y_{j}}\right): \operatorname{Map}_{\mathscr{C}}\left(X_{i}, Y_{j}\right) \rightarrow \operatorname{Map}_{\mathscr{C}}\left(X_{g i}, Y_{g j}\right) .
$$

Definition 1.14 With $X$ and $Y$ as above, set

$$
\operatorname{Map}_{\mathscr{C}_{a}^{I}}(X, Y)=\int_{i} \operatorname{Map}_{\mathscr{C}}\left(X_{i}, Y_{i}\right)
$$

with the $G$-action as described in the diagram $(*)$. 
In other words the mapping space $\operatorname{Map}_{\mathscr{C}_{a}^{I}}(X, Y)$ is the equalizer

$$
\operatorname{Map}_{\mathscr{C}_{a}^{I}}(X, Y) \longmapsto \prod_{i} \operatorname{Map}_{\mathscr{C}}\left(X_{i}, Y_{i}\right) \stackrel{s}{\longrightarrow} \prod_{\alpha: i \rightarrow j} \operatorname{Map}_{\mathscr{C}}\left(X_{j}, Y_{i}\right) .
$$

It is not hard to see that this defines an enrichment of $\mathscr{C}_{a}^{I}$ in $\mathrm{sSet}^{G}$ and that, for each $n \geq 0$, there is an isomorphism of $G$-sets

$$
\operatorname{Map}_{\mathscr{C}_{a}^{I}}(X, Y)_{n} \cong \underline{\mathscr{C}}_{a}^{I}\left(\Delta^{n} \otimes X, Y\right)
$$

Definition 1.15 Let $K: I \rightarrow$ sSet, $L: I^{\mathrm{op}} \rightarrow$ sSet and $X: I \rightarrow \mathscr{C}$ be $G$-diagrams. We set

$$
\begin{gathered}
\operatorname{map}_{I}^{a}(K, X)=\int_{i} \operatorname{map}_{\mathscr{C}}\left(K_{i}, X_{i}\right), \\
L \otimes_{I}^{a} X=\int^{i} L_{i} \otimes X_{i},
\end{gathered}
$$

and give both the $G$-actions from (*).

When $K$ and $L$ are the $G$-diagrams of simplicial sets $N(I /-)$ and $N(-/ I)^{\text {op }}$ from Example 1.3, these constructions specify to the following:

Definition 1.16 For a $G$-diagram $X$ in $\mathscr{C}$, the homotopy limit and homotopy colimit of $X$ are

$$
\underset{I}{\operatorname{holim}} X=\operatorname{map}_{I}^{a}(N(I /-), X) \quad \text { and } \quad \underset{I}{\operatorname{hocolim}} X=N(-/ I)^{\mathrm{op}} \otimes_{I}^{a} X .
$$

These constructions define functors holim and hocolim from $\mathscr{C}_{a}^{I}$ to $\mathscr{C}^{G}$. In the presence of a model structure the words homotopy limit and colimit will always refer to these particular constructions and not, a priori, the derived functors of the limit and colimit, respectively.

Note that there are maps of diagrams $N(-/ I)^{\text {op }} \rightarrow *$ and $N(I /-) \rightarrow *$, where $*$ denotes a chosen one-point simplicial set in both cases. From the formulas above it is easy to see that there are natural isomorphisms $\operatorname{map}_{I}^{a}(*, X) \cong \lim X$ and $X \otimes_{I}^{a} * \cong$ colim $X$. The maps to the terminal diagrams induce equivariant maps

$$
\lim X \rightarrow \operatorname{holim} X \text { and } \operatorname{hocolim} X \rightarrow \operatorname{colim} X .
$$

This paper is in part motivated by the question "when are these maps weak equivalences in $\mathscr{C}^{G}$ ?" 


\subsection{Examples of $G$-diagrams}

In this section we will provide many of the motivating examples for the theory of

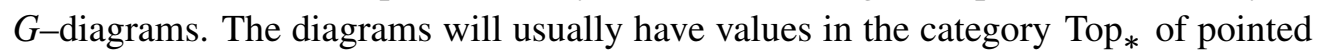
spaces.

For the first two examples we need to fix some notation. Let $Z$ be a pointed space with an action by the finite group $G$. If $T$ is a finite left $G$-set, we write $\mathbb{R}[T]$ for the permutation representation with basis $\left\{e_{t}\right\}_{t \in T}$. The subspace of $\mathbb{R}[T]$ generated by the element $N_{\widetilde{T}} N_{T}=\sum_{t \in T} e_{t}$ is a one-dimensional trivial subrepresentation of $\mathbb{R}[T]$. We define $S^{\widetilde{T}}$ to be the one-point compactification of the orthogonal complement of $\mathbb{R} \cdot N_{T}$ under the usual inner product. We write $\Omega^{\widetilde{T}} Z$ for the $G$-space of continuous pointed maps $\operatorname{Map}_{*}\left(S^{\widetilde{T}}, Z\right)$ with the conjugation action of $G$ and $\Sigma^{\widetilde{T}} Z$ for the smash product $S^{\widetilde{T}} \wedge Z$ with the diagonal $G$-action.

The power set $\mathcal{P}(T)$ inherits a left $G$-action from the action on $T$. The action restricts to the subposet $\mathcal{P}_{0}(T)$ of $\mathcal{P}(T)$ of non-empty sets. Write $\Delta^{\widetilde{T}}$ for the convex hull in $\mathbb{R}[T]$ of the collection of basis vectors $\left\{e_{t}\right\}_{t \in T}$. The barycentric subdivision of $\Delta^{\widetilde{T}}$ is the space $\left|N \mathcal{P}_{0}(T)\right|$ and the standard homeomorphism (see eg [12, Lemma III.4.1]) $h:\left|N \mathcal{P}_{0}(T)\right| \stackrel{\sim}{\longrightarrow} \Delta^{T}$ is equivariant. We fix once and for all an equivariant homeomorphism $i_{T}$ between the interior of the subdivided simplex $\left|N \mathcal{P}_{0}(T)\right|$ and the orthogonal complement $\left(N_{T}\right)^{\perp}$ in $\mathbb{R}[T]$. Together these determine an equivariant homeomorphism

$$
\iota_{T}: S^{\widetilde{T}} \stackrel{\sim}{\longrightarrow}\left|N \mathcal{P}_{0}(T) / \partial\left(N \mathcal{P}_{0}(T)\right)\right| .
$$

Example 1.17 Let $\omega^{\widetilde{T}} Z$ be the $\mathcal{P}_{0}(T)$-indexed $G$-diagram whose value on a subset $U \subseteq T$ is $*$ if $U \neq T$ and $Z$ if $U=T$. The $G$-structure on $\omega^{T} Z$ is given by the action of $G$ on $Z$ at the fixed object $T$ and by the unique maps $* \rightarrow *$ elsewhere in the diagram. We claim that $\iota_{T}$ induces a $G$-homeomorphism

$$
\underset{\mathcal{P}_{0}(T)}{\operatorname{holim}} \omega^{\widetilde{T}} Z \cong \Omega^{\tilde{T}} Z
$$

which is natural in $\underset{\widetilde{T}}{Z}$. Since $\omega^{\widetilde{T}} Z$ has all entries trivial except at the last vertex $T$, we see that holim $\omega^{\widetilde{T}} Z$ is homeomorphic to the subspace in $\operatorname{Map}\left(\left|N \mathcal{P}_{0}(T)\right|, Z\right)$ of maps whose restriction to the boundary is the constant map to the basepoint of $Z$. The map $\iota_{T}$ gives the desired homeomorphism to $\Omega^{\widetilde{T}} Z$ and the naturality is clear.

Example 1.18 Similarly, we let $\mathcal{P}_{1}(T)$ be the $G$-invariant subposet of $\mathcal{P}(T)$ of proper subsets and we define a $G$-diagram $\sigma^{\widetilde{T}} Z$ with value $Z$ at the vertex $\varnothing$ and $*$ elsewhere. The $G$-diagram structure is induced by the $G$-action on $Z$ and the unique 
maps $* \rightarrow *$. A similar argument to the one for $\omega^{\widetilde{T}} Z$ shows that there is a natural $G$-homeomorphism

$$
\underset{\mathcal{P}_{1}(T)}{\operatorname{hocolim}} \sigma^{\widetilde{T}} Z \cong \Sigma^{\widetilde{T}} Z
$$

Example 1.19 More generally, for any pointed category $\mathscr{C}$ and $G$-object $c \in \mathscr{C}^{G}$ define the $\widetilde{T}$-loop space and $\widetilde{T}$-suspension of $c$, respectively, as the pullback and pushout in $\mathscr{C}^{G}$ :
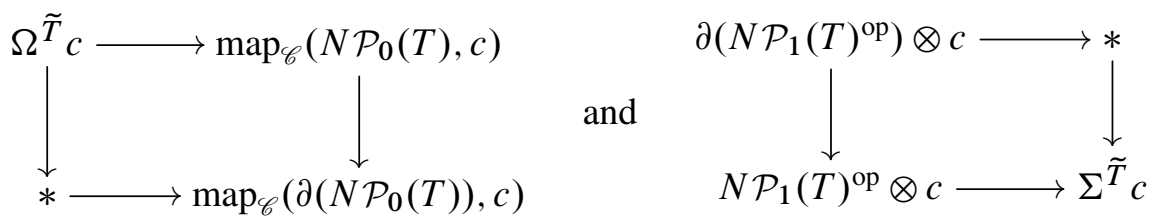

In the case of a pointed $G$-space or $G$-spectra we recover the usual equivariant loop and suspension spaces. These constructions define an adjoint pair of functors $\left(\Sigma^{\widetilde{T}}, \Omega^{\widetilde{T}}\right)$ on $\mathscr{C}^{G}$, by the sequence of natural bijections

$\mathscr{C}^{G}\left(\Sigma^{\tilde{T}} c, d\right)$

$$
\begin{aligned}
& \cong \mathscr{C}^{\mathcal{P}_{1}(2)}\left(\left(N \mathcal{P}_{1}(T)^{\mathrm{op}}\right) \otimes c \leftarrow\left(\partial N \mathcal{P}_{1}(T)^{\mathrm{op}}\right) \otimes c \rightarrow * \otimes c, \Delta d\right) \\
& \cong \mathscr{C}^{\mathcal{P}_{0}(2)}\left(\Delta c, \operatorname{map}_{\mathscr{C}}\left(N \mathcal{P}_{0}(T), d\right) \rightarrow \operatorname{map}_{\mathscr{C}}\left(\partial N \mathcal{P}_{0}(T), d\right) \leftarrow \operatorname{map}_{\mathscr{C}}(*, d)\right) \\
& \cong \mathscr{C}^{G}\left(c, \Omega^{\tilde{T}} d\right) .
\end{aligned}
$$

Here $\Delta c$ and $\Delta d$ are obtained by applying the appropriate diagonal functors to objects of $\mathscr{C}^{G}$. We have used that $* \otimes c=*$ and $\operatorname{map}_{\mathscr{C}}(*, d)=*$, as $\mathscr{C}$ is pointed. Similarly to the previous examples there are natural isomorphisms in $\mathscr{C}^{G}$,

$$
\underset{\mathcal{P}_{0}(T)}{\operatorname{holim}} \omega^{\tilde{T}} c \cong \Omega^{\tilde{T}} c \text { and } \underset{\mathcal{P}_{1}(T)}{\operatorname{hocolim}} \sigma^{\tilde{T}} c \cong \Sigma^{\tilde{T}} c .
$$

Example 1.20 We already saw that for a category $I$ with $G$-action the functor $N(I /-): I \rightarrow$ sSet has an obvious $G$-structure. For a functor $F: I \rightarrow J$ and an object $j$ of $J$, one can form the over-category $F / j$ and the assignment $j \mapsto N(F / j)$ defines a functor $N(F /-): J \rightarrow$ sSet. If $F$ is an equivariant functor between categories with $G$-action there are functors $F / j \rightarrow F /(g j)$ induced by the $G$-actions and, after applying the nerve, these give a $G$-structure on the diagram $N(F /-)$. In fact, $N(F /-)$ with this $G$-structure is the left Kan extension $F_{!} N(I /-)$ of $N(I /-)$ along $F$. This will be important later when we discuss homotopy cofinality and cofibrancy of $G-$ diagrams.

Example 1.21 Let $X: I \rightarrow \mathscr{C}$ be a diagram in a simplicial category $\mathscr{C}$. Define the diagram $q X$ by $q X_{i}=$ hocolim $_{I / i} u_{i}^{*} X$, where $u_{i}: I / i \rightarrow I$ is the functor that forgets 
the map to $i$. A map $\alpha: i \rightarrow j$ in $I$ induces a functor $I / i \rightarrow I / j$ and hence a map $q X_{i} \rightarrow q X_{j}$. The natural map from the homotopy colimit to the colimit induces maps

$$
q X_{i}=\underset{I / i}{\operatorname{hocolim}} u_{i}^{*} X \rightarrow \underset{I / i}{\operatorname{colim}} u_{i}^{*} X \stackrel{\sim}{\longrightarrow} X_{i},
$$

which combine to a map of diagrams $\rho_{X}: q X \rightarrow X$. If $X$ is a $G$-diagram then the functor $I / i \rightarrow I / g i$ induced by multiplication by $g \in G$ induces a map $q X_{i} \rightarrow q X_{g i}$ and, together, these maps constitute a $G$-structure on $q X$. It is a classical fact - see eg [12, Example VII.4.2] for the homotopy limit version - that the objects colim $I q X$ and hocolim $_{I} X$ are isomorphic. In Proposition 2.16(ii) we prove that this isomorphism is $G$-equivariant when $X$ is a $G$-diagram.

\section{$2 G$-diagrams and model structures}

This section provides a framework in which the equivariant constructions of homotopy limits and colimits defined earlier in the paper have homotopical sense and are well behaved. The first step in developing this framework is to give the ambient category $\mathscr{C}$ enough structure to be able to define a model structure on the category of $G$-diagrams in $\mathscr{C}$. It turns out that having a model structure on the category $\mathscr{C}^{G}$ of $G$-objects in $\mathscr{C}$ is not enough; one needs to have homotopical information for all the subgroups of $G$. To encapsulate this information we introduce the notion of a " $G$-model category", which we suggest as a foundation for equivariant homotopy theory.

\subsection{Equivariant model categories}

Let $\mathscr{C}$ be a complete and cocomplete category, $G$ a finite group and $H, H^{\prime} \leq G$ a pair of subgroups. A finite set $K$ with commuting left $H^{\prime}$-action and right $H$-action induces a pair of adjoint functors

$$
K \otimes_{H}(-): \mathscr{C}^{H} \rightleftarrows \mathscr{C}^{H^{\prime}}: \operatorname{hom}_{H^{\prime}}(K,-) .
$$

The left adjoint is defined as

$$
K \otimes_{H} c=\operatorname{colim}\left(H \stackrel{\bigsqcup_{K}^{c}}{\longrightarrow} \mathscr{C}\right),
$$

where $\bigsqcup_{K} c$ is the $H$-equivariant colimit of the constant $H$-diagram $\Delta c$ on the discrete $H$-category $K^{\delta}$ (see Example 1.6) and the $H^{\prime}$-action is induced by the $H^{\prime}$-action on $K$. Dually, define

$$
\operatorname{hom}_{H^{\prime}}(K, d)=\lim \left(H^{\prime} \stackrel{\prod_{K} c}{\longrightarrow} \mathscr{C}\right)
$$


with left $H$-action defined by right action on $K$. These functors are adjoint via the sequence of natural isomorphisms

$$
\begin{aligned}
\mathscr{C}^{H^{\prime}}\left(K \otimes_{H} c, d\right) & \cong \mathscr{C}\left(K \otimes_{H} c, d\right)^{H^{\prime}} \\
& \cong \mathscr{C}^{H}\left(\bigsqcup_{K} c, d\right)^{H^{\prime}} \\
& \cong \mathscr{C}_{a}^{K}\left(\Delta_{K} c, \Delta_{K} d\right)^{H^{\prime}} \\
& \cong \mathscr{C}^{H}\left(c, \prod_{K} d\right)^{H^{\prime}} \\
& \cong \mathscr{C}^{H}\left(c, \lim _{H^{\prime}}\left(\prod_{K} d\right)\right)=\mathscr{C}^{H}\left(c, \operatorname{hom}_{H^{\prime}}(K, d)\right) .
\end{aligned}
$$

We recall the fixed point model structure on $\mathrm{sSet}^{G}$ (see eg [25, Proposition 1.2]). A map $f: X \rightarrow Y$ in $\operatorname{sSet}^{G}$ is a weak equivalence (resp fibration) if for each subgroup $H \leq G$ the map on fixed points $f^{H}: X^{H} \rightarrow Y^{H}$ is a weak equivalence (resp fibration). The cofibrations are the maps which are cofibrations of underlying simplicial sets, ie the levelwise injective maps. In particular, all objects are cofibrant. We will use this model structure unless otherwise stated.

Definition 2.1 A $G$-model category is a cofibrantly generated simplicial model category $\mathscr{C}$ together with the data of a cofibrantly generated model structure on $\mathscr{C}^{H}$ for every subgroup $H \leq G$, satisfying:

(1) The model structure on $\mathscr{C}^{H}$ together with the sSet ${ }^{H}$-enrichment, tensor and cotensor structures induced from $\mathscr{C}$ is a cofibrantly generated $\mathrm{sSet}^{H}$-enriched model structure on $\mathscr{C}^{H}$.

(2) For every pair of subgroups $H, H^{\prime} \leq G$ and finite set $K$ with commuting free left $H^{\prime}$-action and free right $H$-action, the adjunction

$$
K \otimes_{H}(-): \mathscr{C}^{H} \rightleftarrows \mathscr{C}^{H^{\prime}}: \operatorname{hom}_{H^{\prime}}(K,-)
$$

is a Quillen adjunction.

Remark 2.2 For $H^{\prime} \leq H$ and $K=H$ with actions given by left $H^{\prime}$ and right $H$ multiplications, the functor

$$
H \otimes_{H}(-): \mathscr{C}^{H} \longrightarrow \mathscr{C}^{H^{\prime}}
$$

is isomorphic to the functor $\operatorname{res}_{H^{\prime}}^{H}$ that restricts the action. Similarly, for $K=H$ with left $H$ multiplication and right $H^{\prime}$ multiplication, the functor

$$
\operatorname{hom}_{H}(H,-): \mathscr{C}^{H} \longrightarrow \mathscr{C}^{H^{\prime}}
$$


is also isomorphic to the functor $\operatorname{res}_{H^{\prime}}^{H}$. It follows from the second condition that $\operatorname{res}_{H^{\prime}}^{H}$ is both a left and a right Quillen functor. Therefore it preserves cofibrations, acyclic cofibrations, fibrations, acyclic fibrations and equivalences between cofibrant or fibrant objects.

Example 2.3 Let $\mathscr{C}$ be a cofibrantly generated sSet-enriched model category. For a group $H$ the projective ${ }^{1}$ model structure on $\mathscr{C}^{H}$ has as weak equivalences (resp fibrations) the maps whose underlying maps in $\mathscr{C}$ are weak equivalences (resp fibrations). The collection of projective model structures on $\mathscr{C}^{H}$ for $H \leq G$ defines a $G$-model structure on $\mathscr{C}^{G}$. To see this just notice that, if $H^{\prime}$ acts freely on $K$, a choice of section for the quotient map $K \rightarrow H^{\prime} \backslash K$ induces a natural isomorphism

$$
\operatorname{res}_{e}^{H} \operatorname{hom}_{H^{\prime}}(K, c) \cong \prod_{H^{\prime} \backslash K} c,
$$

where $\operatorname{res}_{e}^{H}: \mathscr{C}^{H} \rightarrow \mathscr{C}$ is the forgetful functor. Therefore $\operatorname{hom}_{H^{\prime}}(K,-)$ preserves fibrations and acyclic fibrations.

Example 2.4 Let $\mathscr{C}$ be a cofibrantly generated sSet-enriched model category and fix a pair of finite groups $H \leq G$. For all subgroups $L \leq H$, the $L$-fixed points functor $(-)^{L}: \mathscr{C}^{H} \rightarrow \mathscr{C}$ is defined as the composite

$$
\mathscr{C}^{H} \stackrel{\operatorname{res}_{L}^{H}}{\longrightarrow} \mathscr{C}^{L} \stackrel{\lim }{\longrightarrow} \mathscr{C} .
$$

If these functors are cellular in the sense of [20], the category $\mathscr{C}^{H}$ inherits an sSet ${ }^{H}$ enriched model structure, where weak equivalences and fibrations are the maps that are sent by $(-)^{L}$ to weak equivalences and fibrations in $\mathscr{C}$, respectively, for every subgroup $L \leq H$ (see $[20 ; 26]$ ). This construction specifies to the standard fixed point model structure on (pointed) spaces with $H$-action.

The collection of model categories $\mathscr{C}^{H}$, for $H$ running over the subgroups of $G$, assemble into a $G$-model category. Let us see that the left adjoint $K \otimes_{H}(-)$ is a left Quillen functor. The generating cofibrations of $\mathscr{C}^{H}$ are by definition the images of the generating cofibrations of $\mathscr{C}$ by the functors

$$
J \otimes(-): \mathscr{C} \longrightarrow \mathscr{C}^{H},
$$

where $J$ ranges over finite sets with left $H$-action, and similarly for generating acyclic cofibrations. There is a natural isomorphism

$$
K \otimes_{H}(J \otimes(-)) \cong\left(K \times_{H} J\right) \otimes(-)
$$

\footnotetext{
${ }^{1}$ This is sometimes called the "naïve" model structure on $\mathscr{C}^{H}$. It stands in contrast to "genuine" model structures such as the fixed point model structure on $\mathrm{sSet}^{H}$.
} 
and the right-hand functor preserves cofibrations and acyclic cofibrations, by assumption. Thus $K \otimes_{H}(-)$ preserves generating (acyclic) cofibrations. Since it is a left adjoint, it preserves colimits and therefore all (acyclic) cofibrations (see eg [15, Section 11.2]).

Example 2.5 Let $\mathscr{C}=\mathrm{Sp}^{O}$ be the category of orthogonal spectra and $G$ a finite group. The category $\left(\mathrm{Sp}^{O}\right)^{G}$ of $G$-objects in $\mathrm{Sp}^{O}$ is naturally equivalent to the category of orthogonal $G$-spectra $\mathscr{J}_{G}^{\mathscr{V}} \mathscr{S}$ of [18] indexed on a universe $\mathscr{V}$ for finite-dimensional $G$-representations (see [18, Section V.1; 24, Remark 2.7]). Given any subgroup $H \leq G$, we endow $\left(\mathrm{Sp}^{O}\right)^{H}$ with the model structure induced by the stable model structure

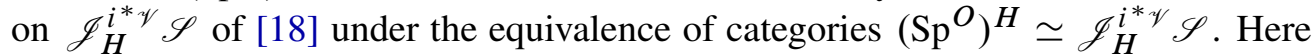
$i: H \rightarrow G$ denotes the inclusion and $i^{*} \mathscr{V}$ is the universe of representations of $H$ that are restrictions of representations of $G$ in $\mathscr{V}$. The adjunctions

$$
K \otimes_{H}(-):\left(\mathrm{Sp}^{O}\right)^{H} \rightleftarrows\left(\mathrm{Sp}^{O}\right)^{H^{\prime}}: \operatorname{hom}_{H^{\prime}}(K,-)
$$

are the standard induction-coinduction adjunctions and they are Quillen adjunctions by [18, Proposition V.2.3]. The collection of model categories $\left\{\left(\mathrm{Sp}^{O}\right)^{H}\right\}_{H \leq G}$ then forms a $G$-model category.

\subsection{The " $G$-projective" model structure on $G$-diagrams}

Let $G$ be a finite group, $\mathscr{C}$ a category and $I$ a small category with $G$-action $a$. Given a $G$-diagram $X$ in $\mathscr{C}_{a}^{I}$ and an object $i \in I$, the vertex $X_{i} \in \mathscr{C}$ inherits from the $G$-structure on $X$ an action by the stabilizer group $G_{i} \leq G$ of the object $i$. This gives an evaluation functor $\mathrm{ev}_{i}: \mathscr{C}_{a}^{I} \rightarrow \mathscr{C}^{G_{i}}$ for every object $i$.

Theorem 2.6 Let $\mathscr{C}$ be a $G$-model category (see Definition 2.1). There is a cofibrantly generated sSet ${ }^{G}$-enriched model structure on the category of $G$-diagrams $\mathscr{C}_{a}^{I}$ with

(1) weak equivalences the maps of $G$-diagrams $f: X \rightarrow Y$ whose evaluations ev i $_{i} f$ are weak equivalences in $\mathscr{C}^{G_{i}}$ for every $i \in I$,

(2) fibrations the maps of G-diagrams $f: X \rightarrow Y$ whose evaluations ev $i f$ are fibrations in $\mathscr{C}^{G_{i}}$ for every $i \in I$,

(3) generating cofibrations and acyclic cofibrations

$$
F \mathcal{I}=\bigcup_{i \in I} F_{i} \mathcal{I}_{i} \quad \text { and } \quad F \mathcal{J}=\bigcup_{i \in I} F_{i} \mathcal{J}_{i}
$$

where $\mathcal{I}_{i}$ and $\mathcal{J}_{i}$ are respectively generating cofibrations and generating acyclic cofibrations of $\mathscr{C}^{G_{i}}$ and $F_{i}: \mathscr{C}^{G_{i}} \rightarrow \mathscr{C}_{a}^{I}$ is the left adjoint to the evaluation functor $\mathrm{ev}_{i}$. 
Remark 2.7 Under the isomorphism $\mathscr{C}_{a}^{I} \cong \mathscr{C}^{G \rtimes_{a} I}$ of Lemma 1.9 the evaluation functor $\mathrm{ev}_{i}$ corresponds to restriction along the functor $\iota_{i}: G_{i} \rightarrow G \rtimes_{a} I$ that sends the unique object to $i$ and a morphism $g$ to $\left(g, \operatorname{id}_{i}: g i=i \rightarrow i\right)$. Since $\mathscr{C}$ has all colimits, a left adjoint for $\mathrm{ev}_{i}$ exists. Also notice that the model structure on $\mathscr{C}_{a}^{I}$ above does not correspond to the projective model structure on $\mathscr{C}^{G \rtimes_{a} I}$.

Before proving the theorem, we need to identify the left adjoints of the evaluation functors. For fixed objects $i, j \in I$, let $K_{j i}$ be the morphisms set

$$
K_{j i}=\operatorname{hom}_{G \rtimes_{a} I}(i, j)=\{(g \in G, \alpha: g i \rightarrow j)\} .
$$

The stabilizer group $G_{j}$ acts freely on the left on $K_{j i}$ by left multiplication on $G$ and by the category action on the morphism component. The group $G_{i}$ acts freely on the right on $K_{j i}$ by right multiplication on the $G$-component. For every $c \in \mathscr{C}^{G_{i}}$ define a diagram $F_{i} c: I \rightarrow \mathscr{C}$ by sending an object $j \in I$ to

$$
\left(F_{i} c\right)_{j}=K_{j i} \otimes_{G_{i}} c .
$$

A morphism $\beta: j \rightarrow j^{\prime}$ in $I$ induces a map $\left(F_{i} c\right)_{j} \rightarrow\left(F_{i} c\right)_{j^{\prime}}$ via the $G_{i}$-equivariant $\operatorname{map} \beta_{*}: K_{j i} \rightarrow K_{j^{\prime} i}$,

$$
\beta_{*}(g, \alpha: g i \rightarrow j)=(g, \beta \circ \alpha) .
$$

The $G_{i}$-equivariant maps $g: K_{j i} \rightarrow K_{(g j) i}$

$$
g\left(g^{\prime}, \alpha: g^{\prime} i \rightarrow j\right)=\left(g g^{\prime}, g \alpha: g g^{\prime} i \rightarrow g j\right)
$$

define a $G$-structure on $F_{i} c$. The construction is clearly functorial in $c$, defining a functor $F_{i}: \mathscr{C}^{G_{i}} \rightarrow \mathscr{C}_{a}^{I}$.

Lemma 2.8 The functor $F_{i}: \mathscr{C}^{G_{i}} \rightarrow \mathscr{C}_{a}^{I}$ is left adjoint to the evaluation functor $\mathrm{ev}_{i}: \mathscr{C}_{a}^{I} \rightarrow \mathscr{C}^{G_{i}}$.

Proof We prove that under the isomorphism $\mathscr{C}_{a}^{I} \cong \mathscr{C}^{G \rtimes_{a} I}$ of Lemma 1.9 the functor $F_{i}$ corresponds to the left Kan extension along the inclusion $\iota_{i}: G_{i} \rightarrow G \rtimes_{a} I$. For an object $j \in I$, the category $\iota_{i} / j$ is the disjoint union of categories

$$
\iota_{i} / j=\coprod_{\substack{z \in G / G_{i} \\ z i \rightarrow j}} E z,
$$

where $E z$ is the translation category of the right $G_{i}$-set $z$, with one object for every element of the orbit $z$ and a unique morphism $h: g \rightarrow g^{\prime}$ whenever $g^{\prime}=g h^{-1}$ for some $h \in G_{i}$. An object $c \in \mathscr{C}^{G_{i}}$ induces a diagram $E c: E z \rightarrow G_{i} \stackrel{c}{\rightarrow} \mathscr{C}$, where the first functor collapses all the objects to the unique object of $G_{i}$ and sends the unique 
morphism $g \rightarrow g h^{-1}$ to $h$. The left Kan extension along $\iota_{i}$ at $c$ is, by definition, the diagram $L_{i} c$ with $j$-vertex

$$
\left(L_{i} c\right)_{j}=\coprod_{\substack{z \in G / G_{i} \\ z i \rightarrow j}} \operatorname{colim} E c .
$$

Notice that the indexing set of the coproduct is precisely the orbit set $K_{j i} / G_{i}$. There is a canonical map of diagrams $F_{i} c \rightarrow L_{j} c$, which at a vertex $j$ is induced by

$$
\coprod_{K_{j i}} c \longrightarrow \coprod_{K_{j i} / G_{i}} \operatorname{colim} E c=\left(L_{i} c\right)_{j}
$$

which on the $(g, \alpha)$-component is the canonical map $c=(E c)_{g} \rightarrow \operatorname{colim}_{E[g]} E c$ to the $[g, \alpha]$-coproduct component. This map respects the $G_{j}$-structure, which on $L_{i} c$ acts on the indexing sets $K_{j i} / G_{i}$. To show that it is an isomorphism, choose a section $s: G / G_{i} \rightarrow G$ for the projection map. This gives a map

$$
\left(L_{i} c\right)_{j}=\coprod_{K_{j i} / G_{i}} \operatorname{colim}_{E z} E c \longrightarrow \coprod_{K_{j i}} c \longrightarrow K_{j i} \otimes_{G_{i}} c=\left(F_{i} c\right)_{j}
$$

that on the $(z, \alpha)$-component is the map induced by $s(z)^{-1} g:(E z)_{g}=c \rightarrow c$ to the $(s(z), \alpha)$-component.

Proof of Theorem 2.6 Weak equivalences and fibrations in $\mathscr{C}_{a}^{I}$ are by definition the morphisms that are sent to weak equivalences and fibrations, respectively, by the functor

$$
\prod_{i \in I} \mathrm{ev}_{i}: \mathscr{C}_{a}^{I} \longrightarrow \prod_{i \in I} \mathscr{C}^{G_{i}}
$$

It follows from Lemma 2.8 that the coproduct of the functors $F_{i}$ defines a left adjoint

$$
F: \prod_{i \in I} \mathscr{C}^{G_{i}} \stackrel{\prod F_{i}}{\longrightarrow} \prod_{i \in I} \mathscr{C}_{a}^{I} \stackrel{\amalg}{\longrightarrow} \mathscr{C}_{a}^{I}
$$

for the product of the evaluation functors. The collections

$$
\mathcal{I}=\bigcup_{i \in I}\left(\mathcal{I}_{i} \times \prod_{j \neq i} \operatorname{id}_{\varnothing_{j}}\right) \quad \text { and } \quad \mathcal{J}=\bigcup_{i \in I}\left(\mathcal{J}_{i} \times \prod_{j \neq i} \operatorname{id}_{\varnothing_{j}}\right)
$$

generate, respectively, the cofibrations and the acyclic cofibrations of $\prod \mathscr{C}^{G_{i}}$ (see eg [15, Proposition 11.1.10]), where $\varnothing_{j}$ is the initial object of $\mathscr{C}^{G_{i}}$. Moreover, their images by $F$ are precisely the families $F \mathcal{I}$ and $F \mathcal{I}$ from the statement. Following [15, Theorem 11.3.2; 26, Remark D.21], we prove: 
(i) $\prod \mathrm{ev}_{j}$ takes relative $F \mathcal{I}$-cell complexes to cofibrations. Let $\lambda$ be a non-zero ordinal and $X: \lambda \rightarrow \mathscr{C}_{a}^{I}$ a functor such that, for all morphisms $\beta \rightarrow \beta^{\prime}$ in $\lambda$, the map $X_{\beta} \rightarrow X_{\beta^{\prime}}$ is a pushout of a map in $F \mathcal{I}$. We need to show that for every $j \in I$ the map

$$
\mathrm{ev}_{j} X_{0} \longrightarrow \mathrm{ev}_{j} \underset{\lambda}{\operatorname{colim}} X=\operatorname{colim}_{\lambda} \mathrm{ev}_{j} \circ X
$$

is a cofibration in $\mathscr{C}^{G_{i}}$. Since ev $\mathrm{ev}_{j}$ commutes with colimits, each map ev $X_{j} X_{\beta} \rightarrow \operatorname{ev}_{j} X_{\beta^{\prime}}$ is the pushout of a map in $\mathrm{ev}_{j} F \mathcal{I}$. Thus we need to show that every map in $\mathrm{ev}_{j} F \mathcal{I}$ is a cofibration of $\mathscr{C}^{G_{j}}$. By definition of $\mathcal{I}$, this is the same as showing that, for all $i, j \in I$, every generating cofibration of $\mathcal{I}_{i}$ is sent by $\mathrm{ev}_{j} F_{i}$ to a cofibration of $\mathscr{C}^{G_{j}}$. The composite functor $\mathrm{ev}_{j} F_{i}$ is, by definition,

$$
\mathrm{ev}_{j} F_{i}=K_{j i} \otimes_{G_{i}}(-): \mathscr{C}^{G_{i}} \longrightarrow \mathscr{C}^{G_{j}},
$$

which sends generating cofibrations to cofibrations as part of the axioms of a $G$-model category (see Definition 2.1).

(ii) $\prod \mathrm{ev}_{j}$ takes relative $F \mathcal{J}$-cell complexes to acyclic cofibrations. The argument is similar to the one above.

Moreover, $\prod \mathrm{ev}_{j}$ preserves colimits. By [15, Theorem 11.3.2; 26, Remark D.21], the families $F \mathcal{I}$ and $F \mathcal{J}$ are respectively a class of generating cofibrations and acyclic cofibrations for the $\mathrm{sSet}^{G}$-enriched model structure on $\mathscr{C}_{a}^{I}$ with the fibrations and weak equivalences of the statement.

Remark 2.9 Recall the isomorphism $\mathscr{C}_{a}^{I} \cong \mathscr{C}^{G \rtimes_{a} I}$ of Lemma 1.9. The model structure on $\mathscr{C}_{a}^{I}$ does not correspond to the projective model structure on $\mathscr{C}^{G \rtimes_{a} I}$. However, every fibration (resp weak equivalence) in $\mathscr{C}_{a}^{I}$ is in paricular a fibration (resp weak equivalence) in $\mathscr{C}^{G \rtimes_{a} I}$. This means that the cofibrations of $\mathscr{C}^{G \rtimes_{a} I}$ are also cofibrations in $\mathscr{C}_{a}^{I}$. In particular, a sufficient condition for an object of $\mathscr{C}_{a}^{I}$ to be cofibrant is to be cofibrant in the projective model structure of $\mathscr{C}^{G \rtimes_{a} I}$.

Proposition 2.10 If $X \in \mathscr{C}_{a}^{I}$ is cofibrant, each vertex $X_{i}$ is cofibrant in $\mathscr{C}^{G_{i}}$.

Proof An argument dual to the proof of Lemma 2.8 shows that the right adjoint $R_{i}$ to the evaluation functor $\mathrm{ev}_{i}: \mathscr{C}_{a}^{I} \rightarrow \mathscr{C}^{G_{i}}$ has $j$-vertex

$$
\mathrm{ev}_{j} R_{i}=\operatorname{hom}_{G_{i}}\left(K_{j i}^{*},-\right) \text {, }
$$

where $K_{j i}^{*}$ is the set $K_{j i}$ with left $G_{i}$-action $g \cdot k:=k \cdot g^{-1}$ and right $G_{j}$-action $k \cdot g:=g^{-1} \cdot k$. Hence $\operatorname{ev}_{j} R_{i}$ is a right Quillen functor by the axioms of a $G-$ model category. Since the fibrations and the equivalences on $\mathscr{C}_{a}^{I}$ are point-wise, 
$R_{i}: \mathscr{C}^{G_{i}} \rightarrow \mathscr{C}_{a}^{I}$ is also a right Quillen functor. It follows that ev ${ }_{i}$ is a left Quillen functor and, in particular, it preserves cofibrant objects.

Definition 2.11 Let $\mathscr{C}$ and $\mathscr{D}$ be $G$-model categories. A $G$-Quillen adjunction (resp equivalence) is an enriched adjunction $\mathscr{C} \rightleftarrows \mathscr{D}$ such that the induced adjunction $\mathscr{C}^{H} \rightleftarrows \mathscr{D}^{H}$ is a Quillen adjunction (resp equivalence) for every subgroup $H \leq G$.

Example 2.12 The Quillen equivalence $|-|$ : sSet $\rightleftarrows$ Top :Sing (see [12, Chapter I]) is a $G-$ Quillen equivalence for any finite group $G$.

Corollary 2.13 A $G$-Quillen equivalence $L: \mathscr{C} \rightleftarrows \mathscr{D}: R$ induces a Quillen equivalence

$$
L: \mathscr{C}_{a}^{I} \rightleftarrows \mathscr{D}_{a}^{I}: R
$$

Proof The adjunction $L: \mathscr{C}_{a}^{I} \rightleftarrows \mathscr{D}_{a}^{I}: R$ is a Quillen adjunction since the right adjoint preserves fibrations and acyclic fibrations, as they are defined point-wise. Let $X \in \mathscr{C}_{a}^{I}$ be cofibrant and $Y \in \mathscr{D}_{a}^{I}$ fibrant. A map $X \rightarrow R(Y)$ is an equivalence if and only if its adjoint $L(X) \rightarrow Y$ is, since by Proposition $2.10 X$ is point-wise cofibrant.

\subsection{Cofibrant replacement of $G$-diagrams}

When $\mathscr{C}$ is a cofibrantly generated simplicial model category and $I$ is a small category, a standard way to replace a diagram $X: I \rightarrow \mathscr{C}$ by a cofibrant diagram is by the construction of Example 1.21. Namely, one defines $q X$ by $q X_{i}=\operatorname{hocolim}_{I / i}\left(u_{i}^{*} X\right)$, where $u_{i}: I / i \rightarrow I$ is the functor that forgets the map to $i$. Then $q X$ is cofibrant in the projective model structure on $\mathscr{C}^{I}$ and the natural map $\rho_{X}: q X \rightarrow X$ is a weak equivalence if $X$ has cofibrant values in $\mathscr{C}$. In this section we will generalize this to $G$-diagrams in a $G$-model category $\mathscr{C}$, for the model structure of Theorem 2.6, as follows:

Theorem 2.14 Let $I$ be a category with a $G$-action. If $X$ is a $G$-diagram such that for all $i$ in $I$ the value $X_{i}$ is cofibrant in $\mathscr{C}^{G_{i}}$, then the map $\rho_{X}: q X \rightarrow X$ is a cofibrant replacement of $G$-diagrams in the sense that $q X$ is cofibrant and $\rho_{X}$ is a weak equivalence.

The proof is technical and will occupy the rest of this section. We begin by fixing some notation. Let $I$ be a small category with an action $a$ of $G$. Write $I^{\delta}$ for the discrete category with the same objects as $I$ but no non-identity morphisms. The inclusion $I^{\delta} \hookrightarrow I$ is equivariant and induces a restriction functor $r: \mathscr{C}_{a}^{I} \rightarrow \mathscr{C}_{a}^{I^{\delta}}$ with left adjoint $r$ ! . We abbreviate $r(X)$ as $X^{\delta}$. Note that the functor $r$ preserves 
fibrations and weak equivalences and hence is a right Quillen functor. It follows that the left adjoint $r_{!}$is a left Quillen functor. We say that an $I$-indexed $G$-diagram $X$ is point-wise cofibrant if for each object $i$ in $I$ the value $X_{i}$ is cofibrant in $\mathscr{C}^{G_{i}}$.

Lemma 2.15 (i) If $Y$ is an $I^{\delta}$-indexed $G$-diagram which is point-wise cofibrant, then $Y$ is cofibrant in $\mathscr{C}_{a}^{I^{\delta}}$.

(ii) In particular, if $X$ is a point-wise cofibrant $I$-indexed G-diagram then $r_{!} X^{\delta}$ is cofibrant in $\mathscr{C}_{a}^{I}$.

Proof To see that part (i) holds, consider a square

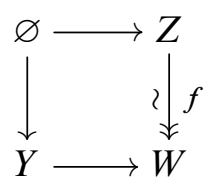

in $\mathscr{C}_{a}^{I^{\delta}}$, where the right-hand vertical map is a trivial fibration and $\varnothing$ denotes the initial object. The map $f$ being a trivial fibration means exactly that each component $f_{i}: Z_{i} \rightarrow W_{i}$ is a trivial fibration in $\mathscr{C}^{G_{i}}$. Choose a representative $i$ of each $G$-orbit in ob $I$. Each resulting square

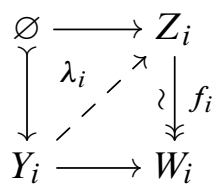

has a lift $\lambda_{i}$ since $Y_{i}$ is cofibrant and $f_{i}$ is a trivial fibration in $\mathscr{C}^{G_{i}}$. For $g \in G$ define $\lambda_{g i}=g_{Z_{i}} \circ \lambda_{i} \circ g_{Y_{i}}^{-1}$. Then, if $g i=i$, the $G_{i}$-equivariance of the map $\lambda_{i}$ says precisely that $\lambda_{i}=g_{Z_{i}} \circ \lambda_{i} \circ g_{Y_{i}}^{-1}=\lambda_{g i}$, so for all $i$ and all $g \in G$ the map $\lambda_{g i}$ is well-defined. It is now easy to see that the $\lambda_{g i}$ assemble to a map of $G$-diagrams giving a lift in the square (5).

Part (ii) follows immediately from part (i) and the fact that $r_{\text {! }}$ is a left Quillen functor and hence preserves cofibrancy of objects.

The adjunction $(r !, r)$ induces a comonad $r ! r$ on $\mathscr{C}_{a}^{I}$ in the usual way. For a $G$-diagram $X$ the value $(r ! r) X$ on $i$ is

$$
(r ! r) X_{i}=\coprod_{\alpha: j \rightarrow i} X_{j}
$$

The counit $\varepsilon:(r ! r) X \rightarrow X$ maps the $X_{j}$-component in the coproduct indexed by $\alpha: j \rightarrow i$ to $X_{i}$ by the map $X(\alpha)$. The comultiplication $c:\left(r_{!} r\right) X \rightarrow\left(r_{!} r r_{!} r\right) X$ has 
as $i$-component the map

$$
\coprod_{\alpha: j \rightarrow i} X_{j} \rightarrow \coprod_{\alpha: j \rightarrow i}\left(\coprod_{\alpha^{\prime}: k \rightarrow j} X_{k}\right)
$$

that maps the $X_{j}$-summand indexed by $\alpha: j \rightarrow i$ by the identity to the $X_{j}$-summand indexed by $\mathrm{id}_{j}$ in the $\alpha$-summand of the target.

Let $X$ be a $G$-diagram indexed on $I$. The bar construction on the comonad $r ! r$ gives a simplicial $G$-diagram $B(r ! r) X$ with $B_{n}(r ! r) X=(r ! r)^{n+1} X$ and therefore

$$
B_{n}(r ! r) X_{i}=\coprod_{\alpha_{0}: i_{0} \rightarrow i} \coprod_{\alpha_{1}: i_{1} \rightarrow i_{0}} \cdots \coprod_{\alpha_{n}: i_{n} \rightarrow i_{n-1}} X_{i_{n}} \cong \coprod_{i_{n} \rightarrow \cdots \rightarrow i_{0} \rightarrow i} X_{i_{n}} .
$$

Note that for varying $n$ the indexing $G_{i}$-simplicial set can be identified with $N(I / i)^{\text {op }}$. For

$$
\sigma=i_{n} \stackrel{\alpha_{n}}{\longrightarrow} \cdots \stackrel{\alpha_{1}}{\longrightarrow} i_{0} \stackrel{\alpha_{0}}{\longrightarrow} i
$$

in $N_{n}(I / i)^{\text {op }}$, the face map $d_{n-k}$ for $k<0$ composes the maps $\alpha_{k}$ and $\alpha_{k-1}$ and $d_{0}$ maps $X_{i_{n}}$ to the $X_{i_{n-1}}$ indexed by $d_{0}(\sigma) \in N_{n-1}(I / i)^{\text {op }}$ by the map $X\left(\alpha_{n}\right)$. The degeneracy map $s_{n}$ inserts an identity in the $(n-l)$-spot. Note that

$$
\underset{I}{\operatorname{colim}} r ! r X=\underset{I^{\delta}}{\operatorname{colim}} r X=\coprod_{i} X_{i}
$$

so that $\operatorname{colim}_{I} B_{n}\left(r_{!} r\right) X \cong \bigsqcup_{\sigma \in N_{n}\left(I^{\mathrm{op}}\right)} X_{\sigma(n)}$ and $\operatorname{colim}_{I} B\left(r_{!} r\right) X$ is isomorphic to the usual simplicial replacement $\bigsqcup_{*} X$ of Bousfield and Kan [5, Section XII.5] with $G$-action induced by the $G$-structure on $X$.

Proposition 2.16 Let $X$ be an $I$-indexed $G$-diagram. Then there are natural isomorphisms in $\mathscr{C}^{G}$ :

(i) $N(-/ I)^{\mathrm{op}} \otimes_{I}^{a} X \cong\left|\coprod_{*} X\right|$.

(ii) $\left|\coprod_{*} X\right| \cong \underset{I}{\operatorname{colim}} q X$.

Proof To see (i) we first decompose the tensor product as an iterated coend (see [22, Section 6.6])

$$
N(-/ I)^{\mathrm{op}} \otimes_{I}^{a} X=\int^{i} N(i / I)^{\mathrm{op}} \otimes X_{i} \cong \int^{i}\left(\int^{[n]} \Delta^{n} \times N_{n}(i / I)^{\mathrm{op}}\right) \otimes X_{i} .
$$


Here and in the rest of the proof we leave it to the reader to check that this is compatible with the $G$-structures on the diagrams. Rearranging the parentheses and switching the order of the coends gives the isomorphic object

$$
\int^{[n]} \int^{i} \Delta^{n} \otimes\left(N_{n}(i / I)^{\mathrm{op}} \otimes X_{i}\right) \cong \int^{[n]} \Delta^{n} \otimes\left(\int^{i} \coprod_{i \rightarrow i_{n} \rightarrow \cdots \rightarrow i_{0}} X_{i}\right) .
$$

Now we analyze the latter $\int^{i}$-factor. It is a coend of the $G$-diagram $I^{\text {op }} \times I \rightarrow \mathscr{C}$ given by

$$
(i, j) \mapsto \coprod_{i \rightarrow i_{n} \rightarrow \cdots \rightarrow i_{0}} X_{j}
$$

This is isomorphic to the diagram

$$
(i, j) \mapsto \coprod_{i_{n} \rightarrow \cdots \rightarrow i_{0}} I\left(i, i_{n}\right) \otimes X_{j}
$$

and we note that since coends commute with colimits there is an isomorphism

$$
\int^{i} \coprod_{i_{n} \rightarrow \cdots \rightarrow i_{0}} I\left(i, i_{n}\right) \otimes X_{i} \cong \coprod_{i_{n} \rightarrow \cdots \rightarrow i_{0}} \int^{i} I\left(i, i_{n}\right) \otimes X_{i} .
$$

Here we must be careful since the representable functor $I\left(-, i_{n}\right)$ is not itself a $G-$ diagram, but the coproduct $\bigsqcup_{\sigma \in N_{n}\left(I^{\mathrm{op}}\right)} I(-, \sigma(n))$ of representable functors is. Finally, we observe that $\int^{i} I\left(i, i_{n}\right) \otimes X_{i} \cong X_{i_{n}}$, so that

$$
\int^{[n]} \Delta^{n} \otimes\left(\int^{i} \coprod_{i \rightarrow i_{n} \rightarrow \cdots \rightarrow i_{0}} X_{i}\right) \cong \int^{[n]} \Delta^{n} \otimes\left(\coprod_{i_{n} \rightarrow \cdots \rightarrow i_{0}} X_{i_{n}}\right)=\left|\coprod_{*} X\right| .
$$

To get the isomorphism in (ii), we recall the isomorphism $\operatorname{colim}_{I} B\left(r_{!} r\right) X \cong \bigsqcup_{*} X$. Since realization commutes with colimits, there are natural isomorphisms

$$
\left|\coprod_{*} X\right| \cong\left|\operatorname{colim}_{I} B(r ! r) X\right| \cong \underset{I}{\operatorname{colim}}|B(r ! r) X| \text {. }
$$

Evaluating at $i$ gives

$$
|B(r ! r) X|_{i}=\left|[n] \mapsto \coprod_{i_{n} \rightarrow \cdots \rightarrow i_{0} \rightarrow i} X_{i_{n}}\right| \cong \underset{I / i}{\operatorname{hocolim}}\left(u_{i}^{*} X\right),
$$

where the last isomorphism is an instance of (i) for the $G_{i}$-diagram $u_{i}^{*} X: I / i \rightarrow \mathscr{C}$. This gives an isomorphism

$$
\underset{I}{\operatorname{colim}}\left|B\left(r_{!} r\right) X\right| \cong \underset{I}{\operatorname{colim}} q X .
$$


Lemma 2.17 If $X$ is a point-wise cofibrant $G$-diagram, then the simplicial object $B\left(r_{!} r\right) X$ is Reedy cofibrant in $\left(\mathscr{C}_{a}^{I}\right)^{\Delta^{\mathrm{op}}}$.

Proof Let $L=L_{n} B\left(r_{!} r X\right)$ be the $n^{\text {th }}$ latching object of $B\left(r_{!} r X\right)$. The natural map

$$
L_{n} B(r ! r X) \rightarrow B_{n}(r ! r X)=B
$$

is at each $i$ in $I$ the inclusion of the summands indexed by the degenerate $n$-simplices in $N_{n}(I / i)^{\text {op }}$ into the coproduct over all $n$-simplices. Thus $B$ decomposes as a coproduct $B=L \amalg N$, where the value of $N$ at $i$ is the coproduct indexed over all the non-degenerate simplices of the nerve. The decomposition is clearly compatible with the $G$-diagram structure on each summand. The diagram $N$ is obtained by applying $r$ ! to a point-wise cofibrant $I^{\delta}$-indexed $G$-diagram and is therefore cofibrant. It follows that the map $L \rightarrow B$ is a cofibration.

Corollary 2.18 If $X$ is a point-wise cofibrant $G$-diagram, then $q X$ is cofibrant.

Proof We know from the proof of Proposition 2.16 that $q X$ is the realization of the simplicial object $B(r ! r) X$, which is Reedy cofibrant by Lemma 2.17 . Since realization takes Reedy cofibrant objects to cofibrant objects [12, Proposition VII.3.6] it follows that $q X$ is cofibrant.

Example 2.19 Let $*_{I}$ be the $I$-indexed $G$-diagram with value the terminal object $*$ of sSet. Then $q\left(*_{I}\right)_{i}=\operatorname{hocolim}_{I / i}\left(*_{I / i}\right) \cong N(I / i)^{\text {op }}$, so that $q\left(*_{I}\right) \cong N(I /-)^{\text {op }}$ and, similarly, $q\left(*_{I \text { op }}\right) \cong N(-/ I)$. By Corollary 2.18 it follows that the diagrams $N(I /-)$ and $N(-/ I)^{\text {op }}$ are cofibrant as $G$-diagrams since $*$ is cofibrant in $\mathrm{sSet}^{G_{i}}$ for all $i$ in $I$ and taking opposite simplicial sets preserves cofibrations. Further, let $I$ and $J$ be categories with respective $G$-actions $a$ and $b$, and $F: I \rightarrow J$ an equivariant functor. Since the left Kan extension $F$ ! preserves cofibrancy, the diagrams $N(F /-) \cong F_{!} N(I /-)$ and $N(-/ F)^{\mathrm{op}} \cong F_{!} N(-/ I)^{\mathrm{op}}$ are also cofibrant in $\mathrm{sSet}_{b}^{J}$.

Proof of Theorem 2.14 It only remains to see that the map $\rho_{X}$ is a weak equivalence. For this we must show that for each $i$ the map $\rho_{X_{i}}$ : hocolim ${ }_{I / i} u_{i}^{*} X \rightarrow X_{i}$ is a weak equivalence in $\mathscr{C}^{G_{i}}$. The functor $\iota: * \rightarrow I / i$ sending the unique object to the terminal object is homotopy cofinal in the sense of Definition 2.24, so by Theorem 2.25 the map $X_{i}=\operatorname{hocolim}_{*} \iota^{*} u_{i}^{*} X \rightarrow \operatorname{hocolim}_{I / i} u_{i}^{*} X$ is a weak equivalence. Since it is also a section to the map $\rho_{X_{i}}$, it follows by the two out of three property that $\rho_{X_{i}}$ is a weak equivalence as well. 


\subsection{Homotopy invariance of map, tensor and homotopy (co)limits}

In this section $\mathscr{C}$ is a $G$-model category in the sense of Definition 2.1 and $a$ is a $G$-action on a small category $I$.

Proposition 2.20 Let $X \in \mathscr{C}_{a}^{I}$ be a $G$-diagram in $\mathscr{C}$. If $X$ is fibrant, the functor

$$
\operatorname{map}_{I}^{a}(-, X):\left(\operatorname{sSet}_{a}^{I}\right)^{\mathrm{op}} \longrightarrow \mathscr{C}^{G}
$$

preserves equivalences of cofibrant objects (in $\operatorname{sSet}_{a}^{I}$ ). Dually, if $X$ is point-wise cofibrant, the functor

$$
(-) \otimes_{I}^{a} X: \mathrm{sSet}_{a}^{I^{\mathrm{op}}} \longrightarrow \mathscr{C}^{G}
$$

preserves equivalences of cofibrant objects.

Proof We prove the statement for map ${ }_{I}^{a}$; the proof for $\otimes_{I}^{a}$ is similar. Let $K \rightarrow L$ be an equivalence of cofibrant diagrams in sSet ${ }_{a}^{I}$. By Ken Brown's lemma we can assume that $K \rightarrow L$ is a cofibration (see [15, Lemma 7.7.1]). To show that the induced map is an equivalence, we need to solve the lifting problem

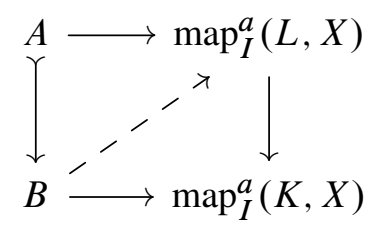

for every cofibration $A \rightarrow B$ in $\mathscr{C}^{G}$. Let $\operatorname{Map}_{\mathscr{C}}(B, X)$ be the $G$-diagram in sSet given by $i \mapsto \operatorname{Map}_{\mathscr{C}}\left(B, X_{i}\right)$, where the $G$-structure is given by the maps

$$
\operatorname{Map}_{\mathscr{C}}\left(g^{-1}, g_{X_{i}}\right): \operatorname{Map}_{\mathscr{C}}\left(B, X_{i}\right) \rightarrow \operatorname{Map}_{\mathscr{C}}\left(B, X_{g}\right) .
$$

The adjunction isomorphism

$$
\underline{\mathscr{C}}^{G}\left(B, \operatorname{map}_{I}^{a}(L, X)\right) \cong \underline{\mathrm{set}}_{a}^{I}\left(L, \operatorname{Map}_{\mathscr{C}}(B, X)\right)
$$

is equivariant and therefore the lifting problem above is equivalent to the lifting problem in $\operatorname{sSet}_{a}^{I}$ :

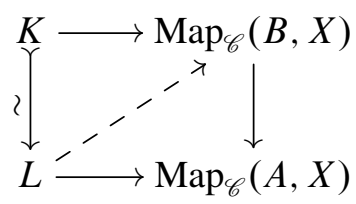

This can be solved if $\operatorname{Map}_{\mathscr{C}}(B, X) \rightarrow \operatorname{Map}_{\mathscr{C}}(A, X)$ is a fibration in $\operatorname{sSet}_{a}^{I}$, ie if for every object $i \in I$ the map $\operatorname{Map}_{\mathscr{C}}\left(B, X_{i}\right) \rightarrow \operatorname{Map}_{\mathscr{C}}\left(A, X_{i}\right)$ is a fibration of simplicial 
$G_{i}$-sets. By assumption, $X_{i}$ is fibrant in $\mathscr{C}^{G_{i}}$ and $A \rightarrow B$ restricts to a cofibration in $\mathscr{C}^{G_{i}}$ so, by axiom SM7 for the $\mathrm{sSet}^{G_{i}}$-enriched model category, $\mathscr{C}^{G_{i}}$ the map is a fibration.

Proposition 2.21 If $K$ is a cofibrant diagram in $\mathrm{sSet}_{a}^{I}$, the functor

$$
\operatorname{map}_{I}^{a}(K,-): \mathscr{C}_{a}^{I} \longrightarrow \mathscr{C}^{G}
$$

preserves equivalences of fibrant objects. Dually if $K$ is cofibrant in $\operatorname{sSet}_{a}^{I^{\text {op }}}$, the functor

$$
K \otimes_{I}^{a}(-): \mathscr{C}_{a}^{I} \longrightarrow \mathscr{C}^{G}
$$

preserves equivalences of point-wise cofibrant objects.

Proof The proof is the same as for the non-equivariant case of [15, Section 18.4], using the equivariant adjunctions as in the proof of Proposition 2.20.

The following result generalizes Villarroel-Flores's result [30, Theorem 6.1]:

Corollary 2.22 Let $\mathscr{C}$ be a $G$-model category, and let $I$ be a category with $G$-action. Then:

(1) holim: $\mathscr{C}_{a}^{I} \rightarrow \mathscr{C}^{G}$ preserves equivalences between fibrant $G$-diagrams.

(2) hocolim: $\mathscr{C}_{a}^{I} \rightarrow \mathscr{C}^{G}$ preserves equivalences between point-wise cofibrant $G$ diagrams.

Proof Recall that homotopy limits and homotopy colimits are defined by cotensoring with $N(I /-)$ and tensoring with $N(-/ I)^{\mathrm{op}}$, respectively. By Proposition 2.21 it is enough to show that $N(I /-)$ is cofibrant in $\operatorname{sSet}_{a}^{I}$ and $N(-/ I)^{\mathrm{op}}$ is cofibrant in $\operatorname{sSet}_{a}^{I^{\text {op }}}$. This was shown in Example 2.19.

For an equivariant functor $F: I \rightarrow J$ between categories with $G$-actions $a$ and $b$, respectively, define the homotopy left Kan extension of a $G$-diagram $X$ in $\mathscr{C}_{a}^{I}$ by

$$
\text { (ho } \left.F_{!} X\right)_{j}=\operatorname{hocolim}(F / j \rightarrow I \stackrel{X}{\longrightarrow} \mathscr{C})
$$

with the induced $G$-structure. The usual homotopy colimit hocolim $I$ is the homotopy left Kan extension along the functor $I \rightarrow *$. Using the simplicial resolution $B(r ! r) X$ of Section 2.3 it is not hard to see that there is a natural isomorphism ho $F_{!} X \cong F_{!}(q X)$. 
Lemma 2.23 (Transitivity of homotopy left Kan extensions) Let $F: I \rightarrow J$ and $F^{\prime}: J \rightarrow K$ be equivariant functors between small categories with $G$-actions $a, b$ and $c$, respectively. If $X$ is a point-wise cofibrant object in $\mathscr{C}_{a}^{I}$ then the natural map

$$
\text { ho } F_{!}^{\prime}(\text { ho } F ! X) \rightarrow \operatorname{ho}\left(F^{\prime} \circ F\right) ! X
$$

is a weak equivalence in $\mathscr{C}_{c}^{K}$. In particular, if $K=*$ then there is a weak equivalence

$$
\underset{J}{\operatorname{hocolim}}\left(\text { ho } F_{!} X\right) \stackrel{\sim}{\rightarrow} \underset{I}{\operatorname{hocolim}} X .
$$

Proof Since $X$ is point-wise cofibrant, the diagram $q X$ is cofibrant and so ho $F_{!} X \cong$ $F_{!} q X$ is cofibrant as well, since $F_{!}$preserves cofibrancy. The functor $F_{!}^{\prime}$ preserves weak equivalences between cofibrant objects, so the natural map $F_{!}^{\prime}\left(q\right.$ ho $\left.F_{!} X\right) \rightarrow F_{!}^{\prime}\left(\right.$ ho $\left.F_{!} X\right)$ is a weak equivalence. The map in the lemma is the composite of the natural maps ho $F_{!}^{\prime}($ ho $F ! X) \stackrel{\sim}{\longrightarrow} F_{!}^{\prime}\left(q\right.$ ho $\left.F_{!} X\right) \stackrel{\sim}{\longrightarrow} F_{!}^{\prime}($ ho $F ! X) \stackrel{\sim}{\longrightarrow} F_{!}^{\prime}\left(F_{!} q X\right) \stackrel{\sim}{\longrightarrow} \operatorname{ho}\left(F^{\prime} \circ F\right) ! X$, where the second map is a weak equivalence by the discussion above.

\subsection{Equivariant cofinality}

Let $I$ and $J$ be categories with respective $G$-actions $a$ and $b, F: I \rightarrow J$ an equivariant functor and $X: J \rightarrow \mathscr{C}$ a $G$-diagram. We want to know when the canonical maps

$\underset{I}{\operatorname{hocolim}} F^{*} X \longrightarrow \underset{J}{\operatorname{hocolim}} X$ and $\underset{J}{\operatorname{holim}} X \longrightarrow \underset{I}{\operatorname{holim}} F^{*} X$

are equivalences in $\mathscr{C}^{G}$. As in the non-equivariant setting, the categories $F / j$ and $j / F$ play a role in answering this question. For every object $j \in J$, these categories inherit a canonical action by the stabilizers group $G_{j} \leq G$ of $j$.

Definition 2.24 The functor $F: I \rightarrow J$ is left (resp right) cofinal if for every $j \in J$ the nerve of the category $F / j(\operatorname{resp} j / F)$ is weakly $G_{j}$-contractible.

Notice that, for $H \leq G_{i}$, the $H$-fixed points of the nerve of $F / j$ are isomorphic to the nerve of $(F / j)^{\bar{H}}$. Therefore $F$ is left cofinal if and only if the fixed categories $(F / j)^{H}$ are contractible for all $H \leq G_{i}$, and similarly for right cofinality. The following cofinality theorem is a generalization of [27, Theorem $1 ; 30$, Theorem 6.3].

Theorem 2.25 Let $\mathscr{C}$ be a $G$-model category, $F: I \rightarrow J$ an equivariant functor and $X \in \mathscr{C}_{b}^{J}$ a $G$-diagram in $\mathscr{C}$. If $F$ is left cofinal and $X$ is fibrant, the canonical map

$$
\underset{J}{\operatorname{holim}} X \longrightarrow \underset{I}{\operatorname{holim}} F^{*} X
$$


is an equivalence in $\mathscr{C}^{G}$. Dually, if $F$ is right cofinal and $X$ is point-wise cofibrant, the map

is an equivalence in $\mathscr{C}^{G}$.

$$
\underset{I}{\operatorname{hocolim}} F^{*} X \longrightarrow \underset{J}{\operatorname{hocolim}} X
$$

Proof We prove the part about left cofinality. The map $\operatorname{holim}_{J} X \rightarrow \operatorname{holim}_{I} F^{*} X$ factors as

$$
\operatorname{map}_{J}^{b}(N J /(-), X) \rightarrow \operatorname{map}_{J}^{b}(N F /(-), X) \stackrel{\sim}{\rightarrow} \operatorname{map}_{I}^{a}\left(N I /(-), F^{*} X\right) .
$$

The second map is a cotensor version of the $\left(F_{!}, F^{*}\right)$-adjunction isomorphism (see Example 1.20). It is equivariant and it is shown to be an isomorphism in [15, Proposition 19.6.6]. The first map is induced by the projection map $N F /(-) \rightarrow N J /(-)$, which is an equivalence in $\operatorname{sSet}_{b}^{J}$ since, for all $H \leq G$ and all objects $j \in J^{H}$, both categories $F / j^{H}$ and $J / j^{H}$ are contractible $\left(J / j^{H}\right.$ has a final object). Moreover, the $G-$ diagrams $N J /(-)$ and $N F /(-)$ are cofibrant in $\operatorname{sSet}_{a}^{J}$, by Example 2.19. Therefore the induced map on mapping objects is an equivalence by the homotopy invariance of $\operatorname{map}_{J}^{b}$ of Proposition 2.20.

As an application of cofinality we prove a "twisted Fubini theorem" for homotopy colimits, describing the homotopy colimit of a $G$-diagram indexed over a Grothendieck construction. The classical version can be found in [6, Proposition 26.5]. Let $I$ be a category with $G$-action and $\Psi \in \mathrm{Cat}_{a}^{I}$ a $G$-diagram of small categories. The Grothendieck construction $I<\Psi$ of the underlying diagram of categories inherits a $G$-action, defined on objects by

$$
g \cdot(i, c \in \mathrm{ob} \Psi(i))=\left(g i, g_{*} c \in \Psi(g i)\right)
$$

and sending a morphism $(\alpha: i \rightarrow j, \gamma: \Psi(\alpha)(c) \rightarrow d)$ from $(i, c)$ to $(j, d)$ to the morphism

$$
g \cdot(\alpha, \gamma)=(g \alpha: g i \rightarrow g j, \Psi(g \alpha)(g c)=g \Psi(\alpha) \stackrel{g \gamma}{\longrightarrow} g d) .
$$

Now let $X \in \mathscr{C}_{a}^{I \imath \Psi}$ be a $G$-diagram in a $G$-model category $\mathscr{C}$. This induces a $G-$ diagram $I \rightarrow \mathscr{C}$ defined at an object $i$ of $I$ by $\left.\operatorname{hocolim}_{\Psi(i)} X\right|_{\Psi(i)}$, where $X$ is restricted along the canonical inclusion $\iota_{i}: \Psi(i) \rightarrow I \prec \Psi$. The $G$-structure is given by the maps

$$
\left.\left.\left.\underset{\Psi(i)}{\operatorname{hocolim}} X\right|_{\Psi(i)} \stackrel{g}{\longrightarrow} \underset{\Psi(i)}{\operatorname{hocolim}} X\right|_{\Psi(g i)} \circ g \stackrel{g_{*}}{\rightarrow} \underset{\Psi(g i)}{\operatorname{hocolim}} X\right|_{\Psi(g i)},
$$

where the first map is induced by the natural transformation of $\Psi(i)$-diagrams

$$
\left.\left.X\right|_{\Psi(i)} \longrightarrow X\right|_{\Psi(g i)} \circ g
$$


provided by the $G$-structure on $X$ and the second map is the canonical map induced by the functor on indexing categories $g: \Psi(i) \rightarrow \Psi(g i)$.

Corollary 2.26 For every point-wise cofibrant $G$-diagram $X \in \mathscr{C}_{a}^{I} \backslash \Psi$ there is a natural equivariant weak equivalence

$$
\eta:\left.\underset{I}{\operatorname{hocolim}} \underset{\Psi(-)}{\operatorname{hocolim}} X\right|_{\Psi(-)} \stackrel{\sim}{\rightarrow} \underset{I / \Psi}{\operatorname{hocolim}} X .
$$

Remark 2.27 When $\mathscr{C}$ is the $G$-model category of spaces with the fixed point model structures and $X: I<\Psi \rightarrow$ Top is the constant one-point diagram the corollary gives a $G$-equivalence

$$
|N(I<\Psi)| \stackrel{\sim}{\longrightarrow} \underset{i \in I}{\operatorname{hocolim}}|N \Psi(i)|
$$

analogous to Thomason's theorem [28]. Our proof is modeled on Thomason's proof.

Proof of Corollary 2.26 Let $p: I \zeta \Psi \rightarrow I$ be the canonical projection. We start by defining a zig-zag of equivalences

$$
\left.\underset{I}{\operatorname{hocolim}} \underset{\Psi(-)}{\operatorname{hocolim}} X\right|_{\Psi(-)} \stackrel{\lambda_{1}}{\longleftarrow} \underset{I}{\operatorname{hocolim}} \text { ho } p_{!} X \stackrel{\lambda_{2}}{\longrightarrow} \underset{I \gtrless \Psi}{\operatorname{hocolim}} X,
$$

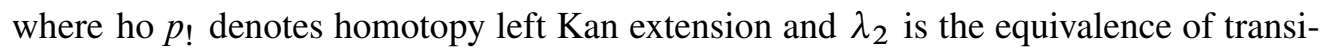
tivity of homotopy left Kan extensions, Lemma 2.23.

For an object $i$ of $I$ define the functor $F_{i}: p / i \rightarrow \Psi(i)$ by $F_{i}(j, c, f: j \rightarrow i)=$ $\Psi(f)(c)$ on objects and on morphisms from $\left(j, c, f_{0}: j \rightarrow i\right)$ to $\left(k, d, f_{1}: k \rightarrow i\right)$ by

$$
F_{i}(h: j \rightarrow k, \alpha: \Psi(h)(c) \rightarrow d)=\Psi\left(f_{1}\right)(\alpha): \Psi\left(f_{0}\right)(c) \rightarrow \Psi\left(f_{1}\right)(d) .
$$

The canonical functor $p / i \rightarrow I<\Psi$ used to define the homotopy left Kan extension (ho $\left.p_{!} X\right)_{i}$ factors as $p / i \stackrel{F_{i}}{\longrightarrow} \Psi(i) \stackrel{\iota_{i}}{\longrightarrow} I<\Psi$. This factorization induces a map $\gamma_{i}$ : (ho $\left.p_{!} X\right)\left._{i} \rightarrow \operatorname{hocolim}_{\Psi(i)} X\right|_{\Psi(i)}$, which is natural in $i$ and compatible with the $G$-structures and hence defines a map of $I$-indexed $G$-diagrams

This induces the map

$$
\gamma: \text { ho }\left.p_{!} X \rightarrow \underset{\Psi(-)}{\operatorname{hocolim}} X\right|_{\Psi(-)}
$$

$$
\lambda_{1}:\left.\underset{I}{\text { hocolim ho }} p_{!} X \longrightarrow \underset{I}{\operatorname{~hocolim}} \underset{\Psi(-)}{\operatorname{hocolim}} X\right|_{\Psi(-)}
$$

in the zig-zag. Let us see that this is an equivalence. For an object $c$ of $\Psi(i)$, the right fiber $c / F_{i}$ has a $\left(G_{i}\right)_{c}$-invariant initial object and is therefore contractible. It follows by cofinality (Theorem 2.25) that the maps $\gamma_{i}$ are weak $G_{i}$-equivalences. By homotopy invariance of homotopy colimits, the induced map $\lambda_{1}$ is a $G$-equivalence. 
It remains to introduce the map $\eta:\left.\operatorname{hocolim}_{I \zeta \Psi} X \rightarrow \operatorname{hocolim}_{I} \operatorname{hocolim}_{\Psi(-)} X\right|_{\Psi(-)}$ from the statement and compare it with the zig-zag. It is defined using the simplicial replacements of Section 2.3. The iterated homotopy colimit hocolim $\operatorname{hocolim}_{\Psi(-)} X_{\Psi(-)}$ is isomorphic to the realization of the simplicial $\mathscr{C}^{G}$-object

$$
[p] \mapsto \coprod_{k_{p} \rightarrow \cdots \rightarrow k_{0}, i_{p} \stackrel{f_{p}}{\rightarrow} \ldots \rightarrow i_{0}} X_{\left(i_{p}, k_{p}\right)}
$$

where the indexing strings of maps are in $N_{p} \Psi\left(i_{p}\right)^{\text {op }}$ and $N_{p} I^{\text {op }}$, respectively. The map $\eta$ in level $p$ maps a summand $X_{\left(i_{p}, k_{p}\right)}$ by the identity map to the summand of

$$
\coprod_{\sigma \in N_{p}(I \curlywedge \Psi)^{\mathrm{op}}} X_{\sigma(p)},
$$

indexed by the $p$-simplex

$$
\left(i_{p}, k_{p}\right) \longrightarrow\left(i_{p-1}, \Psi\left(f_{p}\right)\left(k_{p-1}\right)\right) \longrightarrow \cdots \longrightarrow\left(i_{0}, \Psi\left(f_{p} \cdots f_{1}\right)\left(k_{0}\right)\right)
$$

of $N(I<\Psi)^{\mathrm{op}}$. Just as in Thomason's original proof, there is a simplicial homotopy from $\eta \circ \lambda_{2}$ to $\lambda_{1}$ and it follows that $\eta$ is a weak equivalence (see in particular [28, Lemma 1.2.5]).

\subsection{The Elmendorf theorem for $G$-diagrams}

Let $\mathscr{C}$ be a cofibrantly generated model category with cellular fixed points, in the sense of [20]. Then the category $\mathscr{C}^{G}$ of $G$-object admits the fixed point model structure, where weak equivalences and fibrations are the equivariant maps whose $H$-fixed points are weak equivalences and fibrations in $\mathscr{C}$, respectively, for every subgroup $H \leq G$. Let $\mathcal{O}_{G}$ be the orbit category of $G$, with quotient sets $G / H$ as objects and equivariant maps as morphisms. Elmendorf's theorem (see [26;11]) describes a Quillen equivalence

$$
L: \mathscr{C}^{\mathcal{O}_{G}^{\mathrm{p}}} \rightleftarrows \mathscr{C}^{G}: R,
$$

where the diagram category $\mathscr{C}_{G}^{\text {op }}$ has the projective model structure. In this section we prove an analogous result, giving a Quillen equivalence between the category of $G$-diagrams in $\mathscr{C}$ and a category of diagrams with the projective model structure.

Let $I$ be a small category with an action $a$ of $G$. For convenience we will consider the category of $G$-diagrams in $\mathscr{C}$ as the category $\mathscr{C}^{G \rtimes_{a} I}$ of diagrams indexed over the Grothendieck construction of the action (see Lemma 1.9). The functor $a: G \rightarrow$ Cat induces a functor $\bar{a}: \mathcal{O}_{G}^{\text {op }} \rightarrow$ Cat that sends $G / H$ to the category $I^{H}$ of objects and morphisms of $I$ fixed by the $H$-action. We denote its Grothendieck construction by 
$\mathcal{O}_{G}^{\text {op }} \rtimes_{\bar{a}} I$. The inclusion functor $G \rightarrow \mathcal{O}_{G}^{\text {op }}$ that sends the unique object to $G / 1$ induces a functor $G \rtimes_{a} I \rightarrow \mathcal{O}_{G}^{\mathrm{op}} \rtimes_{\bar{a}} I$, which itself induces a restriction functor

$$
L: \mathscr{C}^{\mathcal{O}_{G}^{\mathrm{op}} \rtimes_{\bar{a}} I} \longrightarrow \mathscr{C}^{G \rtimes_{a} I} .
$$

Recall from Example 2.4 that, if the fixed point functors of $\mathscr{C}$ are cellular, the fixed point model structures on $\mathscr{C}^{H}$ for $H \leq G$ assemble into a $G$-model category.

Theorem 2.28 Let $\mathscr{C}$ be a category such that the fixed points functors for the subgroups of $G$ are cellular. The functor $L: \mathscr{C}_{G}^{\mathcal{O}_{G}^{\mathrm{op}} \rtimes_{\bar{a}} I} \rightarrow \mathscr{C}^{G \rtimes_{a} I}$ is the left adjoint of a Quillen equivalence

$$
L: \mathscr{C}^{\mathcal{O}_{G}^{\mathrm{op}} \rtimes_{\bar{a}} I} \rightleftarrows \mathscr{C}^{G \rtimes_{a} I}: R,
$$

where $\mathscr{C}^{G \rtimes_{a} I}$ has the model structure of Theorem 2.6 and $\mathscr{C}^{\mathcal{O}_{G}^{\mathrm{op}} \rtimes_{\bar{a}} I}$ has the projective model structure.

Proof The right adjoint sends a $G$-diagram $X$ in $\mathscr{C}^{G \rtimes_{a} I} \cong \mathscr{C}_{a}^{I}$ to the diagram $R(X): \mathcal{O}_{G}^{\text {op }} \rtimes_{\bar{a}} I \rightarrow \mathscr{C}$ that sends an object $\left(G / H, i \in I^{H}\right)$ to

$$
R(X)\left(G / H, i \in I^{H}\right)=X_{i}^{H} .
$$

To define $R(X)$ on morphisms, recall that the set of equivariant maps $G / K \rightarrow G / H$ is in natural bijection with $(G / H)^{K}$. A morphism in $\mathcal{O}_{G}^{\text {op }}$ from $(G / H, i)$ to $(G / K, j)$ is a pair $\left(z \in(G / H)^{K},(\alpha: z i \rightarrow j) \in I^{K}\right)$, which is sent to the composite

$$
X_{i}^{H} \stackrel{z}{\longrightarrow} X_{z i}^{K} \stackrel{\alpha_{*}^{K}}{\longrightarrow} X_{j}^{K} .
$$

A morphism $f: X \rightarrow Y$ in $\mathscr{C}_{a}^{I}$ is sent to the natural transformation with value $f_{i}^{H}: X_{i}^{H} \rightarrow Y_{i}^{H}$ at the object $\left(G / H, i \in I^{H}\right)$. It is straightforward to see that $R$ is a right adjoint for $L$. The counit $L R X \rightarrow X$ is an isomorphism and the unit at a diagram $Z$ of $\mathscr{C}_{G}^{\mathcal{O}_{G}^{\mathrm{op}} \rtimes_{\bar{a}} I}$ is the natural transformation

$$
\eta_{Z}: Z(G / H, i) \longrightarrow R L(Z)(G / H, i)=Z(G / 1, i)^{H}
$$

induced by the morphism $\left(H \in(G / H)^{1}, \mathrm{id}_{i}\right):(G / H, i) \rightarrow(G / 1, i)$ of $\mathcal{O}_{G}^{\text {op }} \rtimes_{\bar{a}} I$. By definition of the fixed point model structure and of the model structure on $\mathscr{C} G \rtimes_{a} I$, the right adjoint $R$ preserves and detects equivalences and fibrations. Thus the adjunction $(L, R)$ is a Quillen pair.

Since $R$ preserves and detects equivalences, $(L, R)$ is a Quillen equivalence precisely if the unit $\eta_{Z}: Z \rightarrow R L(Z)$ is an equivalence for all cofibrant objects $Z$ in $\mathscr{C}_{G}^{\mathcal{O}_{G}^{\text {op }} \rtimes \bar{a} I}$. We prove this following the argument of [26]. By cellularity of the fixed point functors, 
$R L$ preserves pushouts along generating cofibrations and directed colimits along pointwise cofibrations. Thus it is enough to show that $\eta_{Z}$ is an isomorphism when $Z$ is a generating cofibrant object, that is, an object of the form

$$
Z=\operatorname{hom}_{\mathcal{O}_{G}^{\mathrm{op}} \rtimes_{\bar{a}} I}((G / H, i),-) \otimes c
$$

for fixed objects $(G / H, i)$ of $\mathcal{O}_{G}^{\text {op }} \rtimes_{\bar{a}} I$ and $c$ of $\mathscr{C}$ cofibrant. For such a $Z$, the unit at an object $(G / K, j)$ is the top horizontal map of the commutative diagram

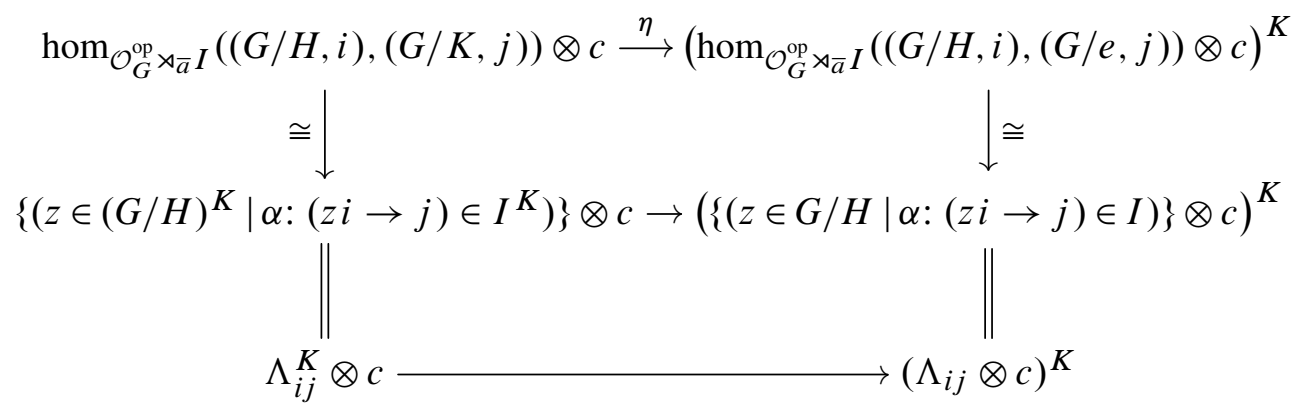

where $\Lambda_{i j}$ is the set of pairs $(z \in G / H, \alpha \in z i \rightarrow j)$ with $K$ acting by left multiplication on $G / H$ and by the category action on the map to $j$ (notice that $j$ belongs to $I^{K}$ ). The bottom horizontal map is an isomorphism by the cellularity conditions on the $K-$ fixed points functor.

For the $G$-model category of spaces, the Elmendorf theorem gives a description of the fixed points of the homotopy limit of a $G$-diagram as a space of natural transformations of diagrams.

Corollary 2.29 For every $G$-diagram of spaces $X$ in $\operatorname{Top}_{a}^{I}$, there is a natural homeomorphism of spaces

$$
\underset{I}{(\operatorname{holim}} X)^{G} \cong \operatorname{Map}_{\operatorname{Top}_{G}^{\mathcal{O}_{G}{ }_{\bar{a}} I}}(R(B I /(-)), R(X)),
$$

where $R(X): \mathcal{O}_{G}^{\text {op }} \rtimes_{\bar{a}} I \rightarrow$ Top has vertices $R(X)_{(G / H, i)}=X_{i}^{H}$.

Proof The space $\left(\operatorname{holim}_{I} X\right)^{G}$ is by definition the mapping space from $B I /(-)$ to $X$ in $\operatorname{Top}_{a}^{I}$. As the counit of the adjunction of the Elmendorf theorem is an isomorphism, there is a sequence of natural homeomorphisms

$$
\begin{aligned}
& \operatorname{Map}_{\operatorname{Top}_{a}^{I}}(B I /(-), X) \cong \operatorname{Map}_{\operatorname{Top}_{a}^{I}}(L R(B I /(-)), X) \\
& \cong \operatorname{Map}_{\operatorname{Top}} \mathcal{O}_{G}^{\text {op }} \rtimes_{\bar{a}} I(R(B I /(-)), R(X)) .
\end{aligned}
$$




\section{Equivariant excision}

We use the homotopy theory of $G$-diagrams developed earlier in the paper to set up a theory of $G$-excisive homotopy functors. Classical excision is formulated using cartesian and cocartesian squares and captures the behavior of homology theories. Blumberg [4] shows that, in the equivariant setting, squares of $G$-objects are not enough to capture the behavior of equivariant homology theories. In the rest of the paper we explain how to replace squares by cubical $G$-diagrams to define a good theory of equivariant excision. We point out that this has already been achieved in [4] in the category of based $G$-spaces. We prove in Proposition 3.31 that our approach and Blumberg's are equivalent in this category.

\subsection{Equivariant cubes}

Let $G$ be a finite group and let $J$ be a finite $G$-set. The poset category of subsets of $J$ ordered by inclusion, $\mathcal{P}(J)$, has a canonical $G$-action, where a group element $g \in G$ sends a subset $U \subset J$ to the set

$$
g \cdot U=\{g \cdot u \mid u \in U\} .
$$

Let $\mathscr{C}$ be a $G$-model category (see Definition 2.1).

Definition 3.1 The category of $J$-cubes in $\mathscr{C}$ is the category of $G$-diagrams $\mathscr{C}_{a}^{\mathcal{P}(J)}$ for the action $a$ on $\mathcal{P}(J)$ described above.

In order to define a homotopy invariant notion of (co)cartesian cubes, we need to make our homotopy (co)limits homotopy invariant. Given a cube $X \in \mathscr{C}_{a}^{\mathcal{P}(J)}$ let $F X$ denote a fibrant $J$-cube (in the model structure of Theorem 2.6) together with an equivalence $X \stackrel{\sim}{\rightarrow} F X$. Similarly, let $Q X \stackrel{\sim}{\longrightarrow} X$ denote an equivalence with $Q X$ point-wise cofibrant, that is, with $Q X_{U}$ cofibrant in $\mathscr{C}^{G_{U}}$ for every $U \in \mathcal{P}(J)$.

Remark 3.2 To find a replacement $F X$, one can simply use the fibrant replacement in the model category $\mathscr{C}_{a}^{\mathcal{P}(J)}$. Similarly, a cofibrant replacement $Q X$ in $\mathscr{C}_{a}^{\mathcal{P}(J)}$ is in particular point-wise cofibrant by Proposition 2.10. However, for a given cube one can often find a more explicit point-wise cofibrant replacement that is not necessarily cofibrant in $\mathscr{C}_{a}^{\mathcal{P}(J)}$ (see Examples 3.4 and 3.5 below). For example, if a functorial cofibrant replacement $Q$ in $\mathscr{C}$ lifts to a cofibrant replacement in $\mathscr{C}^{H}$ for every $H \leq G$, the diagram $Q X$ is point-wise cofibrant. 
Let us denote by $\mathcal{P}_{0}(J)$ the full subcategory of $\mathcal{P}(J)$ on the objects different from the empty set and let $\mathcal{P}_{1}(J)$ be the full subcategory of $\mathcal{P}(J)$ of proper subsets of $J$. Since $\varnothing$ and $J$ are fixed by the $G$-action, $\mathcal{P}_{0}(J)$ and $\mathcal{P}_{1}(J)$ inherit a $G$-action. We let $\iota_{0}: \mathcal{P}_{0}(J) \rightarrow \mathcal{P}(J)$ and $\iota_{1}: \mathcal{P}_{1}(J) \rightarrow \mathcal{P}(J)$ be the corresponding inclusions.

Definition 3.3 Let $\mathscr{C}$ be a $G$-model category and $J$ a finite $G$-set. A $J$-cube $X \in \mathscr{C}_{a}^{\mathcal{P}(J)}$ is homotopy cocartesian if the canonical map

$$
\underset{\mathcal{P}_{1}(J)}{\operatorname{hocolim}} \iota_{1}^{*} Q X \longrightarrow Q X_{J} \stackrel{\sim}{\rightarrow} X_{J}
$$

is an equivalence in $\mathscr{C}^{G}$. Dually, $X \in \mathscr{C}_{a}^{\mathcal{P}(J)}$ is homotopy cartesian if the canonical map

$$
X_{\varnothing} \stackrel{\sim}{\longrightarrow} F X_{\varnothing} \longrightarrow \operatorname{holim}_{\mathcal{P}_{0}(J)} \iota_{0}^{*} F X
$$

is an equivalence in $\mathscr{C}^{G}$. We will drop the inclusions from the notation when convenient.

Example 3.4 Let $J$ be a finite $G$-set and let $J_{+}$be the $G$-set $J$ with an added disjoint fixed basepoint. For a cofibrant object $c \in \mathscr{C}^{G}$, define a $J_{+}-$cube $S^{J} c$ with vertices

$$
\left(S^{J} c\right)_{U}= \begin{cases}c & U=\varnothing, \\ C^{U} c & U \lesseqgtr J_{+}, \\ \Sigma^{J} c & U=J_{+} .\end{cases}
$$

Here $\Sigma^{J} c=\Sigma^{\widetilde{J}_{+}} c$ is the suspension by the permutation representation of $J$ defined in Example 1.19 and $C^{U} c$ denotes the $U$-iterated cone

$$
C^{U} c=\underset{\mathcal{P}(U)}{\operatorname{hocolim}}\left(S \longmapsto\left\{\begin{array}{ll}
c & \text { if } S=\varnothing \\
* & \text { otherwise }
\end{array}\right) \simeq * .\right.
$$

Since $c$ is cofibrant, $S^{J} c$ is point-wise cofibrant. Let us prove that it is homotopy cocartesian. Its restriction to $\mathcal{P}_{1}\left(J_{+}\right)$is the cofibrant replacement $q$ of Theorem 2.14 for the diagram $\sigma^{J} c: \mathcal{P}_{1}\left(J_{+}\right) \rightarrow \mathscr{C}$ with $\left(\sigma^{J} c\right)_{\varnothing}=c$ and with the terminal object at the other vertices. Since homotopy colimits and colimits agree on cofibrant objects (by the homotopy invariance of $\otimes_{I}^{a}$ ), the canonical map from the homotopy colimit factors as the equivalence

$$
\underset{\mathcal{P}_{1}\left(J_{+}\right)}{\operatorname{hocolim}} S^{J} c=\underset{\mathcal{P}_{1}\left(J_{+}\right)}{\operatorname{hoccolim}} q\left(\sigma^{J} c\right) \stackrel{\sim}{\rightarrow} \underset{\mathcal{P}_{1}\left(J_{+}\right)}{\operatorname{colim}} q\left(\sigma^{J} c\right) \cong \underset{\mathcal{P}_{1}\left(J_{+}\right)}{\operatorname{hocolim}} \sigma^{J} c=\Sigma^{J} c .
$$

Example 3.5 Suppose that $\mathscr{C}$ has a zero object $*$ and let us denote the coproduct of $\mathscr{C}$ by $\bigvee$. Let $c$ be a cofibrant object of $\mathscr{C}^{G}$ and let $J$ be a finite $G$-set. Define a 
$J$-cube $W^{J} c$ with vertices

$$
\left(W^{J} c\right)_{U}= \begin{cases}\bigvee_{J} c & U=\varnothing, \\ c & |U|=1, \\ * & |U| \geq 2,\end{cases}
$$

with initial map $\left(W^{J} c\right)_{\varnothing}=\bigvee_{J} c \rightarrow c=\left(W^{J} c\right)_{\{j\}}$ the pinch map that collapses every wedge summand different from $j$. This has a $G$-structure defined by the action on $\bigvee_{J} c$ on the initial vertex and by the action maps $g:\left(W^{J} c\right)_{\{j\}}=c \rightarrow c=\left(W^{J} c\right)_{\{g j\}}$. The cube $W^{J} c$ is homotopy cocartesian, that is, its homotopy colimit over $\mathcal{P}_{1}(J)$ is equivalent in $\mathscr{C}^{G}$ to the zero object. To see this, we replace $W^{J} c$ by the equivalent cube

$$
(\bar{W} c)_{U}= \begin{cases}\bigvee c & U=\varnothing, \\ c \underset{J \backslash j}{\bigvee} C c & U=\{j\}, \\ \bigvee_{J} C c & |U| \geq 2,\end{cases}
$$

where $C c$ is the one-fold cone $C c=\operatorname{hocolim}(c \rightarrow *)$ and the non-identity maps of the diagram are all induced by cone inclusions $c \rightarrow C c$. The $G$-structure is defined similarly as before, by permuting the wedge components. The cube $\bar{W} c$ is cofibrant, since the latching maps are all equivariant cofibrations (see Proposition A.6). As homotopy colimits preserve equivalences of point-wise cofibrant diagrams, we get equivalences

$$
\underset{\mathcal{P}_{1}(J)}{\operatorname{hocolim}} W^{J} c \stackrel{\sim}{\rightarrow} \underset{\mathcal{P}_{1}(J)}{\operatorname{hocolim}} \bar{W} c \stackrel{\sim}{\rightarrow} \operatorname{colim}_{\mathcal{P}_{1}(J)} \bar{W} c \cong \bigvee_{J} C c
$$

This is contractible, since $\bigvee_{J}$ is a left Quillen functor, and therefore it preserves equivalences of cofibrant objects.

\subsection{Equivariant homotopy functors}

Given two $G$-model categories $\mathscr{C}$ and $\mathscr{D}$, we define the notion of "homotopy functor" from $\mathscr{C}$ to $\mathscr{D}$. We shall consider functors for which we can express compatibility conditions with the model structures on $\mathscr{C}^{H}$ and $\mathscr{D}^{H}$ for every subgroup $H \leq G$. These are the functors $\Phi: \mathscr{C} \rightarrow \mathscr{D}^{G}$.

These functors are precisely the functors for which the formation of $G$-diagrams is functorial, in the sense that a functor $\Phi: \mathscr{C} \rightarrow \mathscr{D}^{G}$ induces a functor $\Phi_{*}: \mathscr{C}_{a}^{I} \rightarrow \mathscr{D}_{a}^{I}$ for any category with $G$-action $I$. Given a $G$-diagram $X$ in $\mathscr{C}_{a}^{I}$, the $G$-structure on the diagram $\Phi_{*}(X)=\Phi \circ X$ is defined by the maps

$$
\Phi\left(X_{i}\right) \stackrel{g}{\longrightarrow} \Phi\left(X_{i}\right) \stackrel{\Phi(g)}{\longrightarrow} \Phi\left(X_{g i}\right) .
$$


Since each map $\Phi(g)$ is $G$-equivariant, $\Phi(g) g=g \Phi(g)$ and our choice of applying $g$ first is irrelevant. When $I=*$ is the trivial category, this functor is the classical extension $\Phi: \mathscr{C}^{G} \rightarrow \mathscr{D}^{G}$. Similarly, the functor $\Phi: \mathscr{C} \rightarrow \mathscr{D}^{H}$ obtained by restricting the $G$-action to a subgroup $H$ of $G$ extends to a functor $\Phi: \mathscr{C}^{H} \rightarrow \mathscr{D}^{H}$.

Definition 3.6 We call $\Phi: \mathscr{C} \rightarrow \mathscr{D}^{G}$ a homotopy functor if for every subgroup $H$ of $G$ the extended functor $\Phi: \mathscr{C}^{H} \rightarrow \mathscr{D}^{H}$ preserves equivalences of cofibrant objects. In particular, the induced functor $\Phi_{*}: \mathscr{C}_{a}^{I} \rightarrow \mathscr{D}_{a}^{I}$ preserves equivalences of point-wise cofibrant $G$-diagrams.

Example 3.7 The following are all examples of functors $\mathscr{C}^{G} \rightarrow \mathscr{D}^{G}$ that are extensions of homotopy functors $\mathscr{C} \rightarrow \mathscr{D}^{G}$ :

- The identity functor $\mathscr{C}^{G} \rightarrow \mathscr{C}^{G}$.

- The functors $K \wedge(-), \operatorname{Map}_{*}(K,-)$ : $\operatorname{Top}_{*}^{G} \rightarrow \operatorname{Top}_{*}^{G}$ for a fixed well-pointed $G$-space $K$.

- The functor $E \wedge(-): \operatorname{Top}_{*}^{G} \rightarrow\left(\mathrm{Sp}^{O}\right)^{G}$ for a fixed orthogonal $G$-spectrum $E$.

An example of a functor $\mathscr{C}^{G} \rightarrow \mathscr{D}^{G}$ that is not the extension of a functor $\mathscr{C} \rightarrow \mathscr{D}^{G}$ is the quotient functor $(-) / G$ : $\operatorname{Top}^{G} \rightarrow \operatorname{Top}^{G}$ that sends a $G$-space to its orbit space with the trivial $G$-action.

Let $\Lambda: \Phi \rightarrow \Phi^{\prime}$ be a natural transformation of functors $\Phi, \Phi^{\prime}: \mathscr{C} \rightarrow \mathscr{D}^{G}$. For every subgroup $H$ of $G$, the natural transformation $\Lambda$ induces a natural transformation $\Lambda_{H}$ of functors $\mathscr{C}^{H} \rightarrow \mathscr{D}^{H}$ from the extension of $\Phi$ to the extension of $\Phi^{\prime}$.

Definition 3.8 A natural transformation $\Lambda: \Phi \rightarrow \Phi^{\prime}$ is an equivalence if, for every subgroup $H$ of $G$ and every object $c$ of $\mathscr{C}^{H}$, the morphism $\Lambda_{H}: \Phi(c) \rightarrow \Phi^{\prime}(c)$ is an equivalence in $\mathscr{D}^{H}$.

In Section 3.5 we are going to consider enriched functors, which have the advantage of admitting assembly maps. We recall that a $G$-model category $\mathscr{C}$ is by default enriched over the category sSet of simplicial sets, and that this induces $H$-sSet-enrichments on $\mathscr{C}^{H}$ for every subgroup $H$ of $G$ compatible with the given model structures. When a functor $\Phi: \mathscr{C} \rightarrow \mathscr{D}^{G}$ is enriched over sSet, its extension $\Phi: \mathscr{C}^{H} \rightarrow \mathscr{D}^{H}$ is automatically enriched over $H$-sSet. That is, if $b$ and $c$ are objects of $\mathscr{C}^{H}$, the map of simplicial sets

$$
\Phi: \operatorname{Map}_{\mathscr{C}}(b, c) \longrightarrow \operatorname{Map}_{\mathscr{C}}(\Phi(b), \Phi(c))
$$

is $H$-equivariant with respect to the conjugation actions. In particular, the functor $\Phi: \mathscr{C}^{H} \rightarrow \mathscr{D}^{H}$ preserves homotopy equivalences and therefore equivalences between fibrant and cofibrant objects. 
Remark 3.9 Waldhausen [31, Lemmas 3.1.2-3.1.3] proves that any sufficiently nice homotopy functor on the category of simplicial sets is equivalent to an enriched functor. Biedermann and Röndigs [3] extend Waldhausen's result to simplicial model categories. A similar statement holds in our equivariant context. Let $\Phi: \mathscr{C} \rightarrow \mathscr{D}^{G}$ be a homotopy functor and suppose that, for every $H \leq G$ and every object $c$ in $\mathscr{C}^{H}$, the simplicial object

$$
\Phi\left(\operatorname{map}_{\mathscr{C}}\left(\Delta^{(-)}, c\right)\right): \Delta^{\mathrm{op}} \longrightarrow \mathscr{D}^{H}
$$

in the model category $\mathscr{D}^{H}$ is Reedy cofibrant. Then the functor $\Phi^{\Delta}: \mathscr{C} \rightarrow \mathscr{D}^{G}$ defined as the geometric realization

$$
\Phi^{\Delta}(c)=\left(\coprod_{n} \Delta^{n} \otimes \Phi\left(\operatorname{map}_{\mathscr{C}}\left(\Delta^{n}, c\right)\right)\right) / \sim
$$

is sSet-enriched and the natural map $\Phi \rightarrow \Phi^{\Delta}$ is an equivalence. This is proved in [3, Lemma 4.2].

Given a simplicial $G$-set $K$ and an object $c$ of $\mathscr{C}^{G}$, there is a $G$-equivariant assembly map

$$
A_{K}: K \otimes \Phi(c) \longrightarrow \Phi(K \otimes c)
$$

in $\mathscr{D}^{G}$. It is natural both in the $G$-object $c$ and in the simplicial $G$-set $K$. It is defined as the adjoint to the map of simplicial $G$-sets

$$
K \longrightarrow \operatorname{Map}_{\mathscr{C}}(c, K \otimes c) \stackrel{\Phi}{\longrightarrow} \operatorname{Map}_{\mathscr{D}}(\Phi(c), \Phi(K \otimes c)),
$$

where the first map is adjoint to the identity of $K \otimes c$. If $\mathscr{C}$ and $\mathscr{D}$ are pointed, these maps induce a map

$$
\alpha: \Phi(c) \longrightarrow \Omega^{J} \Phi\left(\Sigma^{J} c\right)
$$

in $\mathscr{D}^{G}$, called the adjoint assembly map, which is defined as follows. Recall from Example 1.19 the definitions of $\Omega^{J}$ and $\Sigma^{J}$, and let us write $\Delta^{J}=N\left(\mathcal{P}_{0}\left(J_{+}\right)\right)$. The assembly maps for the simplicial $G$-sets $\Delta^{J}$ and $\partial \Delta^{J}$ and the pushout square defining $\Sigma^{J}$ give a commutative diagram:

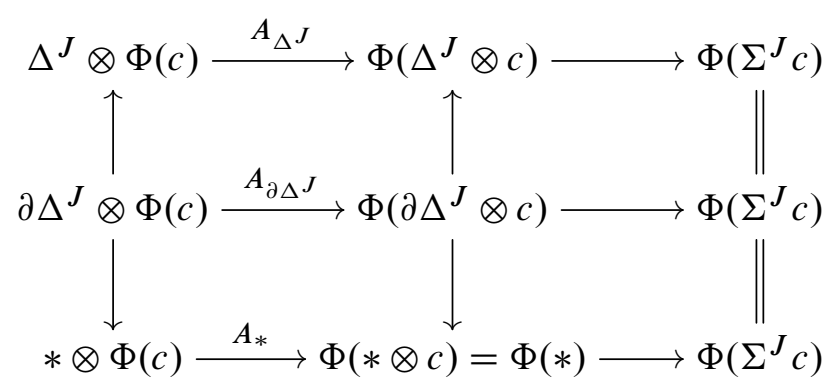


By adjoining the tensor in the compositions of the horizontal maps we obtain a commutative square:

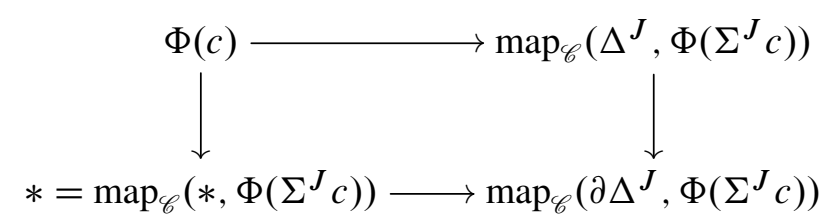

The adjoint assembly map $\alpha: \Phi(c) \rightarrow \Omega^{J} \Phi\left(\Sigma^{J} c\right)$ is the canonical map from the initial vertex of this square into the pullback of the rest of the diagram. We will see in Section 3.5 how this map is intimately related to the $G$-excision of $\Phi$.

Remark 3.10 Let $\mathscr{B}$ and $\mathscr{D}$ be simplicial model categories and let $\mathscr{C}$ be a small full subcategory of $\mathscr{B}$. In [3, Theorem 4.14] the authors prove that, under certain conditions on the model structures, the category of enriched functors $\mathscr{C} \rightarrow \mathscr{D}$ admits a model structure where the equivalences are point-wise and the fibrant objects are precisely the homotopy functors. We wonder if a similar amount of hard work would show that, if $\mathscr{B}$ and $\mathscr{D}$ are well-behaved $G$-model categories, the category of enriched functors $\mathscr{C} \rightarrow \mathscr{D}^{G}$ admits a model structure with the equivalences of Definition 3.8 where the fibrant objects are the homotopy functors of Definition 3.6.

\section{3 $G$-excision}

Let $G$ be a finite group and let $\Phi: \mathscr{C} \rightarrow \mathscr{D}^{G}$ be a homotopy functor between $G$ model categories. We saw in Section 3.2 that $\Phi$ induces a functor between $G_{+}$-cubes $\Phi_{*}: \mathscr{C}_{a}^{\mathcal{P}\left(G_{+}\right)} \rightarrow \mathscr{D}_{a}^{\mathcal{P}\left(G_{+}\right)}$.

Definition 3.11 We say that a homotopy functor $\Phi: \mathscr{C} \rightarrow \mathscr{D}^{G}$ is $G$-excisive if $\Phi_{*}: \mathscr{C}_{a}^{\mathcal{P}\left(G_{+}\right)} \rightarrow \mathscr{D}_{a}^{\mathcal{P}\left(G_{+}\right)}$sends homotopy cocartesian $G_{+}$-cubes to homotopy cartesian $G_{+}$-cubes. When $\mathscr{C}$ and $\mathscr{D}$ are pointed, we say that $\Phi$ is $G$-linear if it is $G$-excisive and $\Phi(*)$ is equivalent to the zero object in $\mathscr{D}^{G}$.

The choice of indexing the cubes on the $G$-set $G_{+}$seems arbitrary at first sight. We justify and explain this choice, including the extra basepoint added to $G$, in Proposition 3.13 and Remark 3.15 below.

Example 3.12 The following are examples of $G$-linear homotopy functors, as we will see later in the paper. 
- Let $M$ be an abelian group with additive $G$-action. Consider the homotopy functor $M(-): \mathrm{sSet}_{*} \rightarrow \mathrm{sSet}_{*}^{G}$ that sends a simplicial set $Z$ to

$$
M(Z)_{n}=\bigoplus_{z \in Z_{n}} M z / M *
$$

where $G$ acts diagonally on the direct summands. We show in Example 3.33 that this functor is $G$-linear and explain how this is related to the equivariant EilenbergMac Lane spectrum $H M$ being a fibrant orthogonal $G$-spectrum. The homotopy groups of the extension of $M(-)$ to $\mathrm{sSet}_{*}^{G}$ are the Bredon homology of the Mackey functor $H \mapsto M^{H}$.

- For a fixed orthogonal $G$-spectrum $E$ in $\left(\mathrm{Sp}^{O}\right)^{G}$, the homotopy functor

$$
E \wedge(-): \mathrm{Top}_{*} \rightarrow\left(\mathrm{Sp}^{O}\right)^{G}
$$

is $G$-linear (see Proposition 3.36). The stable homotopy groups of the extension of $E \wedge(-)$ to pointed $G$-spaces is the equivariant homology theory associated to $E$.

- The inclusion of spectra with trivial $G$-action $\mathrm{Sp}^{O} \rightarrow\left(\mathrm{Sp}^{O}\right)^{G}$ (which extends to the identity on $G$-spectra) is $G$-linear (see Theorem 3.35).

The next result shows that our choice of indexing the cubes on the $G$-set $G_{+}$in the definition of $G$-excision plays a minor role; we could equivalently have indexed the cubes on transitive $G$-sets with disjoint basepoints.

Proposition 3.13 A homotopy functor $\Phi: \mathscr{C} \rightarrow \mathscr{D}^{G}$ is $G$-excisive if and only if the induced functor $\Phi_{*}: \mathscr{C}_{a}^{\mathcal{P}\left(G / H_{+}\right)} \rightarrow \mathscr{D}_{a}^{\mathcal{P}\left(G / H_{+}\right)}$sends homotopy cocartesian $\mathrm{G} / \mathrm{H}_{+}-$ cubes to homotopy cartesian $G / H_{+}-$cubes for every subgroup $H \leq G$.

Remark 3.14 Setting $H=G$ in Proposition 3.13 we see that $\Phi_{*}: \mathscr{C}_{a}^{\mathcal{P}\left(1_{+}\right)} \rightarrow \mathscr{D}_{a}^{\mathcal{P}\left(1_{+}\right)}$ sends cocartesian squares in $\mathscr{C}^{G}$ to cartesian squares in $\mathscr{D}^{G}$. That is, if $\Phi$ is $G$-excisive then the induced functor $\Phi_{*}: \mathscr{C}^{G} \rightarrow \mathscr{D}^{G}$ is excisive in the classical sense.

Proof of Proposition 3.13 The "if" part of the statement is trivial. For the "only if" part, let $H$ be a subgroup of $G$ and consider the projection map $p: G_{+} \rightarrow G / H_{+}$. As part of a broader discussion on how to calculate homotopy limits and colimits of punctured cubes, we show in Propositions A.1 and A.3 that the induced restriction functor $p^{*}: \mathscr{C}_{a}^{\mathcal{P}\left(G / H_{+}\right)} \rightarrow \mathscr{C}_{a}^{\mathcal{P}\left(G_{+}\right)}$preserves homotopy cocartesian cubes and detects homotopy cartesian cubes. Therefore, given a homotopy cocartesian cube $X$ in $\mathscr{C}_{a}^{\mathcal{P}\left(G / H_{+}\right)}$, the cube $p^{*} X$ in $\mathscr{C}_{a}^{\mathcal{P}\left(G_{+}\right)}$is homotopy cocartesian and, by $G$-excision of $\Phi$, the cube $\Phi_{*}\left(p^{*} X\right)=p^{*} \Phi_{*}(X)$ is homotopy cartesian in $\mathscr{D}_{a}^{\mathcal{P}\left(G_{+}\right)}$. As $p^{*}$ detects homotopy cocartesian cubes, $\Phi_{*}(X)$ is homotopy cartesian in $\mathscr{D}_{a}^{\mathcal{P}\left(G / H_{+}\right)}$. 
Remark 3.15 The basepoint added to $G$ in the definition of $G$-excision (Definition 3.11) has the role of combining in a single condition the behavior of $\Phi: \mathscr{C} \rightarrow \mathscr{D}^{G}$ on squares and on $G$-cubes. We already saw (Remark 3.14) that if $\Phi$ is $G$-excisive it sends homotopy cocartesian squares to homotopy cartesian squares. It turns out that $\Phi_{*}: \mathscr{C}_{a}^{\mathcal{P}(G / H)} \rightarrow \mathscr{D}_{a}^{\mathcal{P}(G / H)}$ also turns homotopy cocartesian $G / H$-cubes into homotopy cartesian ones. This can be proved by extending a $\mathrm{G} / \mathrm{H}$-cube to a $\mathrm{G} / \mathrm{H}_{+}-$ cube by means of the functor $p: \mathcal{P}\left(G / H_{+}\right) \rightarrow \mathcal{P}(G / H)$ that intersects a subset with $G / H$, with a proof analogous to Proposition 3.13. Conversely, similar techniques show that if $\Phi: \mathscr{C} \rightarrow \mathscr{D}^{G}$ turns homotopy cocartesian squares and $G$-cubes into homotopy cartesian ones, it is $G$-excisive.

Remark 3.16 The $G$-linearity of functors $\Phi: \mathscr{C} \rightarrow \mathscr{D}^{G}$ from a pointed $G$-model category $\mathscr{C}$ is hereditary with respect to taking subgroups, under a mild assumption on the target $G$-model category $\mathscr{D}$. That is to say, if $\Phi$ is $G$-linear it is also $H$-linear for every subgroup $H$ of $G$. The proof we suggest requires a surprising amount of machinery and it is given in Corollary 3.23 as a corollary of a higher Wirthmüller isomorphism theorem. It is still unknown to the authors if in the unpointed case $G$-excision satisfies a similar property.

Proposition 3.17 Let $\mathscr{C}$ and $\mathscr{D}$ be pointed $G$-model categories and $\Phi: \mathscr{C} \rightarrow \mathscr{D}^{G}$ be a $G$-linear homotopy functor. For any finite $G$-set $J$ and any cofibrant $G$-object $c \in \mathscr{C}^{G}$, the canonical map

$$
\Phi\left(\bigvee_{J} c\right) \rightarrow \prod_{J} F \Phi(c)
$$

is an equivalence in $\mathscr{D}^{G}$.

Proof First assume that $J=1_{+}$with trivial $G$-action. The square $V c$

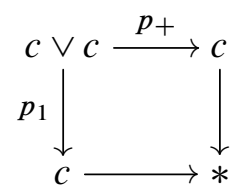

in $\mathscr{C}^{G}$ is homotopy cocartesian (see Example 3.5). By Remark 3.14 its image $\Phi(V c)$ is homotopy cartesian, that is, the map

$$
\Phi(c \vee c) \stackrel{\sim}{\longrightarrow} F \Phi(c \vee c) \rightarrow \operatorname{holim}_{\mathcal{P}_{0}\left(1_{+}\right)} F \Phi(V c) \cong F \Phi(c) \times F \Phi(c)
$$

is a weak equivalence in $\mathscr{D}^{G}$, with diagonal action on the target. By induction, the map of the statement is an equivalence for every $J$ with trivial $G$-action. Given a finite 
$G$-set $J$, decompose it as the disjoint union of transitive $G$-sets $J=\bigsqcup_{z \in G \backslash J} z$. The map of the statement decomposes as

$$
\Phi\left(\bigvee_{J} c\right)=\Phi\left(\bigvee_{z \in G \backslash J} \bigvee_{z} c\right) \stackrel{\sim}{\rightarrow} \prod_{z \in G \backslash J} F \Phi\left(\bigvee_{z} c\right) \rightarrow \prod_{z \in G \backslash J} \prod_{z} F \Phi(c)=\prod_{J} F \Phi(c)
$$

with the first map an equivalence as the action on the quotient $G \backslash J$ is trivial. Therefore it is enough to show that the map is an equivalence for $J=G / H$ a transitive $G$-set.

Consider the $G / H_{+}-$cube $W c$ with vertices

$$
(W c)_{U}= \begin{cases}\bigvee_{G / H} c & U=\varnothing \\ c & U=\{j \neq+\} \\ * & \text { otherwise. }\end{cases}
$$

It is homotopy cocartesian by an argument completely similar to Example 3.5. By Proposition 3.13 the cube $\Phi(W c)$ is homotopy cartesian, that is, the canonical map

$$
\Phi\left(\bigvee_{G / H} c\right) \rightarrow \operatorname{holim}_{\mathcal{P}_{0}\left(G / H_{+}\right)} F \Phi(W c) \cong \prod_{G / H} F \Phi(c)
$$

is an equivalence in $\mathscr{D}^{G}$.

Remark 3.18 In this equivariant setting $G_{+}$-cubes (or equivalently $J_{+}$-cubes for $J$ transitive) play the role that squares play in the classical theory. The equivariant analogue of $n$-cubes should be cubes indexed on $G$-sets with $n$ distinct $G$-orbits and a disjoint basepoint. Following [13], the behavior of $\Phi$ on these cubes should be related to higher-order $G$-excision. This will be the subject of a later article.

\subsection{The generalized Wirthmüller isomorphism theorem}

Let $\mathscr{C}$ be a bicomplete category and let $H$ and $H^{\prime}$ be subgroups of a finite group $G$. We recall from Section 2.1 that a finite set $K$ with commuting left $H^{\prime}$-action and right $H$-action induces an adjunction

$$
K \otimes_{H}(-): \mathscr{C}^{H} \rightleftarrows \mathscr{C}^{H^{\prime}}: \operatorname{hom}_{H^{\prime}}(K,-) .
$$

Let $K^{*}$ be the set $K$ with left $H$-action and right $H^{\prime}$-action defined by $h \cdot k \cdot h^{\prime}=$ $\left(h^{\prime}\right)^{-1} \cdot k \cdot h^{-1}$. If $\mathscr{C}$ has a zero-object $*$ and the actions on $K$ are free, a functor $\Phi: \mathscr{C} \rightarrow \mathscr{D}^{G}$ induces a natural transformation

$$
\eta: \Phi\left(K \otimes_{H}(-)\right) \longrightarrow \operatorname{hom}_{H}\left(K^{*}, \Phi(-)\right)
$$


of functors $\mathscr{C}^{H} \rightarrow \mathscr{D}^{H^{\prime}}$. The map $\eta_{c}$ is the image by the composition

$$
\begin{aligned}
\mathscr{C}^{H}\left(\left(K^{*} \times_{H^{\prime}} K\right) \otimes_{H} c, c\right) \stackrel{\Phi}{\longrightarrow} \mathscr{D}^{H}\left(\Phi\left(\left(K^{*} \times_{H^{\prime}} K\right) \otimes_{H} c\right), \Phi(c)\right) \\
\quad \rightarrow \mathscr{D}^{H}\left(K^{*} \otimes_{H^{\prime}} \Phi\left(K \otimes_{H} c\right), \Phi(c)\right) \stackrel{\sim}{\longrightarrow} \mathscr{D}^{H^{\prime}}\left(\Phi\left(K \otimes_{H} c\right), \operatorname{hom}_{H}\left(K^{*}, \Phi(c)\right)\right)
\end{aligned}
$$

of the map $\bigvee_{K^{*} \times_{H^{\prime}} K} c \rightarrow c$ defined by $h: c \rightarrow c$ on a $\left(k, k^{\prime}\right)$-component with $k^{\prime} h=k$ and by the trivial map $c \rightarrow * \rightarrow c$ otherwise. Notice that since the $H$-action is free there is at most one $h$ for which $k^{\prime} h=k$.

Example 3.19 Suppose that $K=G=H^{\prime}$ with left $G$-multiplication and right $H$-multiplication. Sending an element to its inverse defines an $H-G$-equivariant isomorphism between $G^{*}$ and $G$ with left $H$-multiplication and right $G$-multiplication. We saw in Remark 2.2 that the forgetful functor $\mathscr{C}^{G} \rightarrow \mathscr{C}^{H}$ is right adjoint to $G \otimes_{H}(-)$ and left adjoint to $\operatorname{hom}_{H}\left(G^{*},-\right)$. The map $\eta$ for the identity functor is the standard map

$$
G \otimes_{H}(-) \longrightarrow \operatorname{hom}_{H}\left(G^{*},-\right),
$$

which in the case of spectra is the classical Wirthmüller isomorphism map. In Theorem 3.35 we apply Theorem 3.20 below to recover the Wirthmüller isomorphism theorem for $G$-spectra.

Theorem 3.20 Let $\mathscr{C}$ and $\mathscr{D}$ be pointed $G$-model categories and suppose that $K$ admits an $H^{\prime}-H$-equivariant map to $G$; this happens eg if $K=G$. For every $G$-linear homotopy functor $\Phi: \mathscr{C} \rightarrow \mathscr{D}^{G}$ and every object $c$ in $\mathscr{C}^{H}$, the composite

$$
\Phi\left(K \otimes_{H} c\right) \stackrel{\eta_{c}}{\longrightarrow} \operatorname{hom}_{H}\left(K^{*}, \Phi(c)\right) \longrightarrow \operatorname{hom}_{H}\left(K^{*}, F \Phi(c)\right)
$$

is an equivalence in $\mathscr{D}^{H^{\prime}}$, where $\Phi(c) \stackrel{\sim}{\longrightarrow} F \Phi(c)$ is a fibrant replacement of $\Phi(c)$ in $\mathscr{D}^{H}$. In particular, if the right Quillen functor $\operatorname{hom}_{H}\left(K^{*},-\right)$ preserves all weak equivalences, the map $\eta_{c}: \Phi\left(K \otimes_{H} c\right) \rightarrow \operatorname{hom}_{H}\left(K^{*}, \Phi(c)\right)$ is a weak equivalence for any $c \in \mathscr{C}^{H}$.

Proof We express the map of the statement as a canonical map into the homotopy limit of a punctured cube, then we use the $G$-linearity of $\Phi$ to conclude that the map is an equivalence. For this we will compare the source and target of $\eta$ with an indexed coproduct and product, respectively. 
Choose a section $s_{G}: G / H \rightarrow G$ and an $H^{\prime}-H$-equivariant map $\phi: K \rightarrow G$. These choices give a commutative diagram (of sets)

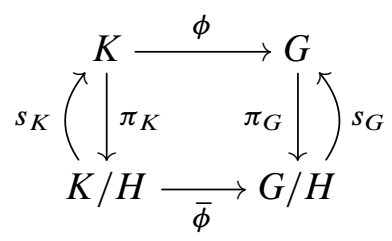

where $s_{K}(k H):=k \cdot\left(\phi(k)^{-1} \cdot s_{G} \pi_{G} \phi(k)\right)$ is a section for $\pi_{K}$, satisfying the relation $\phi s_{K}=s_{G} \bar{\phi}$. This gives a map $\gamma: H^{\prime} \times K / H \rightarrow H$ defined by

$$
\gamma\left(h^{\prime}, z\right)=s_{G}\left(h^{\prime} \bar{\phi}(z)\right)^{-1} \cdot h^{\prime} \cdot s_{G} \bar{\phi}(z),
$$

which we use to define functors $\bigvee_{K / H}(-): \mathscr{C}^{H} \rightarrow \mathscr{C}^{H^{\prime}}$ and $\prod_{K / H}(-): \mathscr{D}^{H} \rightarrow \mathscr{D}^{H^{\prime}}$. These send objects $c$ and $d$ to the coproduct $\bigvee_{K / H} c$ and product $\prod_{K / H} d$, respectively, with $H^{\prime}$-actions ${ }^{2}$

$$
h^{\prime} \cdot(z, x)=\left(h^{\prime} z, \gamma\left(h^{\prime}, z\right) \cdot x\right) \quad \text { and } \quad\left(h^{\prime} \cdot \underline{y}\right)_{z}=\gamma\left(h^{\prime}, z\right) \cdot y_{\left(h^{\prime}\right)^{-1} z},
$$

respectively. There is a commutative diagram of natural transformations

$$
\begin{aligned}
& \Phi\left(\bigvee_{K / H} c\right) \longrightarrow \bigvee_{K / H} \Phi(c) \longrightarrow \prod_{K / H} \Phi(c) \\
& \Phi\left(s_{K} \otimes \mathrm{id}_{c}\right) \downarrow \cong \quad s_{K} \otimes \mathrm{id}_{\Phi(c)} \downarrow \cong \quad \cong \downarrow(-) \circ s_{K} \\
& \eta: \Phi\left(K \otimes_{H} c\right) \longrightarrow K \otimes_{H} \Phi(c) \longrightarrow \operatorname{hom}_{H}\left(K^{*}, \Phi(c)\right) \text {. }
\end{aligned}
$$

The top-right horizontal map is the canonical map from the coproduct to the product. The first two vertical maps are induced by the composite

$$
s_{K} \otimes \mathrm{id}: \bigvee_{K / H} c=K / H \otimes c \rightarrow K \otimes c \rightarrow K \otimes_{H} c .
$$

This is an isomorphism with inverse $(k, x) \mapsto\left(\pi_{K} k,\left(s_{G} \pi_{G} \phi(k)\right)^{-1} \phi(k) \cdot x\right)$. The right vertical map (-) $\circ s_{K}$ is defined dually and it is also an isomorphism. We can therefore equivalently study the top composition $\Phi\left(\bigvee_{K / H} c\right) \rightarrow \prod_{K / H} \Phi(c)$.

Consider the $K / H_{+}-$cube $W c: \mathcal{P}\left(K / H_{+}\right) \rightarrow \mathscr{C}$ defined by

$$
(W c)_{S}= \begin{cases}\bigvee_{K / H} c & S=\varnothing, \\ c & |S|=1, S \neq\{+\}, \\ * & |S| \geq 2 \text { or } S=\{+\},\end{cases}
$$

${ }^{2}$ For convenience we only spell these actions out in the case that the objects of $\mathscr{C}$ have "elements". 
with initial map $\bigvee_{K / H} c \rightarrow c=(W c)_{\{z\}}$ the pinch map that collapses all the wedge components not indexed by $\{z\}$. The structure maps $c=(W c)_{z} \rightarrow(W c)_{h^{\prime} z}=c$ are defined by $\gamma\left(h^{\prime}, z\right) \in H$.

The cube $W c$ is homotopy cocartesian. Indeed, if $Q_{H} c \stackrel{\sim}{\rightarrow} c$ is a cofibrant replacement of $c$ in $\mathscr{C}^{H}$, the cube $W Q_{H} c$ is point-wise cofibrant with homotopy colimit over $\mathcal{P}_{1}\left(K / H_{+}\right)$contractible (see Example 3.5). Let $\Phi(W c) \stackrel{\sim}{\longrightarrow} F \Phi(W c)$ be a fibrant replacement of $\Phi(W c)$. By linearity of $\Phi$, the canonical map

$$
\Phi\left(\bigvee_{K / H} c\right) \stackrel{\sim}{\rightarrow} \operatorname{holim}_{\mathcal{P}_{0}\left(K / H_{+}\right)} F \Phi(W c) \cong \prod_{K / H} F \Phi(c)
$$

is an equivalence in $\mathscr{D}^{H^{\prime}}$. This proves the first part of the theorem.

Moreover, the map above fits into a commutative diagram

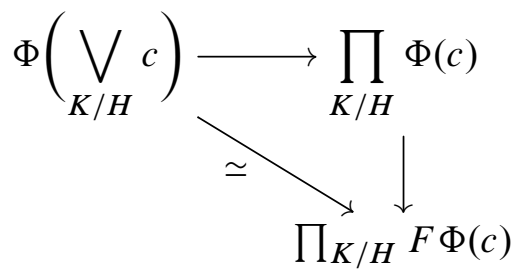

where the right vertical map is an equivalence if the functor $\operatorname{hom}_{H}\left(K^{*},-\right)$ (and therefore $\left.\prod_{K / H}(-)\right)$ preserves weak equivalences.

Corollary 3.21 If the trivial action inclusion functor $\mathscr{C} \rightarrow \mathscr{C}^{G}$ is G-linear, the left and right adjoints to the evaluation functor $\mathrm{ev}_{i}: \mathscr{C}_{a}^{I} \rightarrow \mathscr{C}^{G_{i}}$ are naturally equivalent on fibrant objects for every $i \in I$.

Proof We saw in Lemma 2.8 that the left adjoint $F_{i}: \mathscr{C}^{\boldsymbol{G}_{i}} \rightarrow \mathscr{C}_{a}^{I}$ has $j$-vertex

$$
\left(F_{i} c\right)_{j}=K_{j i} \otimes_{G_{i}} c,
$$

where $K_{j i}=\operatorname{hom}_{G \rtimes_{a} I}(i, j)$ projects $G_{j}-G_{i}$-equivariantly to $G$. Similarly the right adjoint has $j$-vertex

$$
\left(R_{i} c\right)_{j}=\operatorname{hom}_{G_{i}}\left(K_{j i}^{*}, c\right)
$$

and Theorem 3.20 provides a natural equivalence from $F_{i}$ to $R_{i}$.

We give a "higher version" of the Wirthmüller isomorphism theorem, which compares the left and the right adjoints of the functor on $J$-cubes that restricts the action to a subgroup $H$ of $G$. Given a $G$-set $J$, let $\left.J\right|_{H}$ be the $H$-set obtained by restricting the 
$G$-action to $H$. The poset category with $H$-action $\mathcal{P}\left(\left.J\right|_{H}\right)$ is the category $\mathcal{P}(J)$ with the restricted action $\left.a\right|_{H}$. There is a forgetful functor $\mathscr{C}_{a}^{\mathcal{P}(J)} \rightarrow \mathscr{C}_{\left.a\right|_{H}}^{\mathcal{P}\left(\left.J\right|_{H}\right)}$ that restricts the $G$-structure to an $H$-structure. It has both a left and a right adjoint, which we denote respectively $L^{J}$ and $R^{J}$. This can easily be seen with the description of $G$-diagrams as diagrams on a Grothendieck construction of Lemma 1.9, because the restriction functor above corresponds to restriction along the inclusion $\iota: H \rtimes_{\left.a\right|_{H}} \mathcal{P}\left(\left.J\right|_{H}\right) \rightarrow G \rtimes_{a} \mathcal{P}(J)$. The following result specializes to Theorem 3.20 for $K=G$ when $J$ is the empty $G$-set.

Theorem 3.22 For every $G$-linear homotopy functor $\Phi: \mathscr{C} \rightarrow \mathscr{D}^{G}$ and $\left.J\right|_{H^{-c u b e}}$ $X \in \mathscr{C}_{\left.a\right|_{H}}^{\mathcal{P}\left(\left.J\right|_{H}\right)}$, there is an equivalence of $J$-cubes

$$
\Phi L^{J}(X) \stackrel{\eta}{\longrightarrow} R^{J} \Phi(X) \longrightarrow R^{J} F \Phi(X),
$$

where $\Phi(X) \stackrel{\sim}{\rightarrow} F \Phi(X)$ is a fibrant replacement of $\Phi(X)$.

Proof Let us describe the left adjoint $L^{J}$ explicitly, by calculating the left Kan extension of $X$ along $\iota: H \rtimes_{\left.a\right|_{H}} \mathcal{P}\left(\left.J\right|_{H}\right) \rightarrow G \rtimes_{a} \mathcal{P}(J)$. By definition, this has values

$$
L^{J}(X)_{U}=\operatorname{colim}\left(\iota / U \rightarrow H \rtimes_{\left.a\right|_{H}} \mathcal{P}\left(\left.J\right|_{H}\right) \stackrel{X}{\longrightarrow} \mathscr{C}\right) .
$$

The over-category $\iota / U$ is the poset with objects $\left(g \in G, A \in \mathcal{P}\left(g^{-1} U\right)\right)$ and a unique morphism $(g, A) \rightarrow\left(g^{\prime}, A^{\prime}\right)$ whenever $g\left(g^{\prime}\right)^{-1}$ belongs to $H$ and $g\left(g^{\prime}\right)^{-1} A \subset A^{\prime}$. This can be written as the disjoint union of categories

$$
\iota / U=\coprod_{z \in G / H}\left(E z \prec \Psi_{z}\right),
$$

where $E z$ is the translation category of the right $H$-set $z$ (see Lemma 2.8) and $E z \prec \Psi_{z}$ is the Grothendieck construction of the functor $\Psi_{z}: E z \rightarrow$ Cat that sends $g \in G / H$ to the category $\mathcal{P}\left(g^{-1} U\right)$. Hence the left Kan extension $L^{J}(X)$ is naturally isomorphic to

$$
\begin{aligned}
L^{J}(X)_{U} & \cong \bigvee_{z \in G / H} \operatorname{colim}_{(g, A) \in E z \imath \Psi_{z}} X_{A} \\
& \cong \bigvee_{z \in G / H} \operatorname{colim}_{g \in E z} \operatorname{colim}_{A \in \mathcal{P}\left(g^{-1} U\right)} X_{A} \simeq \bigvee_{z \in G / H} \operatorname{colim}_{g \in E z} X_{g^{-1} U}
\end{aligned}
$$

Here the first isomorphism is the Fubini theorem for colimits (see eg [6, Proposition 40.2]; as it is an isomorphism it is enough to see that it is equivariant). The last map is an isomorphism because $g^{-1} U$ is a terminal object in $\mathcal{P}\left(g^{-1} U\right)$. A choice of section $s: G / H \rightarrow G$ gives a further identification

$$
L^{J}(X)_{U} \cong \bigvee_{z \in G / H} X_{S(z)^{-1} U}
$$


Chasing through the isomorphisms one can see that the $G$-structure is given by the maps

$$
g: X_{s(z)^{-1} U} \stackrel{s(g z)^{-1} g s(z)}{\longrightarrow} X_{s(g z)^{-1} g U}
$$

The same choice of section gives a similar identification for the right adjoint,

$$
R^{J}(X)_{U} \cong \prod_{z \in G / H} X_{s(z)^{-1} U}
$$

A $G / H_{+}-$cube argument completely analogous to Theorem 3.20 shows that the inclusion of wedges into products induces a $G$-equivalence $\Phi L^{J}(X) \rightarrow R^{J} F \Phi(X)$.

Corollary 3.23 Let $J$ be a finite $G$-set and $H$ be a subgroup of $G$. Let $\Phi: \mathscr{C} \rightarrow \mathscr{D}^{G}$ be a homotopy functor and suppose that $\operatorname{hom}_{H}(G,-): \mathscr{D}^{H} \rightarrow \mathscr{D}^{G}$ detects equivalences of fibrant objects. If $\Phi_{*}: \mathscr{C}_{a}^{\mathcal{P}(J)} \rightarrow \mathscr{D}_{a}^{\mathcal{P}(J)}$ sends homotopy cocartesian cubes to homotopy cartesian cubes, so does $\Phi_{*}: \mathscr{C}_{\left.a\right|_{H}}^{\mathcal{P}\left(\left.J\right|_{H}\right)} \rightarrow \mathscr{D}_{\left.a\right|_{H}}^{\mathcal{P}\left(\left.J\right|_{H}\right)}$. It follows that, if $\Phi$ is $G$-linear, it is also $H$-linear for every subgroup $H$ of $G$.

Proof From the explicit descriptions of $L^{J}$ and $R^{J}$ of Theorem 3.22, one can see that $L^{J}$ commutes with homotopy colimits and that $R^{J}$ commutes with homotopy limits. In particular, if $X$ is a homotopy cocartesian $\left.J\right|_{H^{-}}$-cube, the $J$-cube $L^{J}(X)$ is also homotopy cocartesian. Hence, by our assumption on $\Phi$, the $J$-cube $\Phi_{*} L^{J}(X)$ is homotopy cartesian. The top horizontal map in the commutative diagram

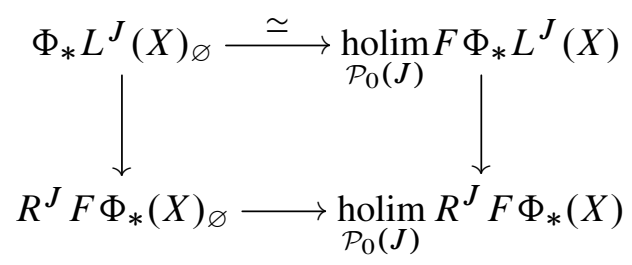

is therefore an equivalence. The vertical maps are also equivalences by the higher Wirthmüller isomorphism theorem, Theorem 3.22. Thus the bottom horizontal map is also an equivalence and it factors as

$$
R^{J} F \Phi_{*}(X)_{\varnothing} \rightarrow R^{\varnothing} \operatorname{holim}_{\mathcal{P}_{0}(J)} F \Phi_{*}(X) \stackrel{\sim}{\rightarrow} \operatorname{holim}_{\mathcal{P}_{0}(J)} R^{J} F \Phi_{*}(X) .
$$

The first map of the factorization is therefore also an equivalence and, by the explicit description of $R^{J}$ in the proof of Theorem 3.22, it is just the canonical map

$$
\operatorname{hom}_{H}\left(G, F \Phi_{*}(X)_{\varnothing}\right) \longrightarrow \operatorname{hom}_{H}\left(G, \operatorname{holim}_{\mathcal{P}_{0}(J)} F \Phi_{*}(X)\right) .
$$

Since $\operatorname{hom}_{H}(G,-)$ detects equivalences of fibrant objects, $\Phi_{*}(X)$ is homotopy cartesian. 
For the second part of the statement, assume that $\Phi$ is $G$-linear and let $X$ be a homotopy cocartesian $H_{+}-$cube. Consider the $H$-equivariant surjection $p:\left.G_{+}\right|_{H} \rightarrow H_{+}$which is the identity on $H$ and that collapses the complement of $H$ to the basepoint. It induces a functor $p^{*}: \mathscr{C}_{a}^{\mathcal{P}\left(H_{+}\right)} \rightarrow \mathscr{C}_{a}^{\mathcal{P}\left(\left.G_{+}\right|_{H}\right)}$, which, by Proposition A.3, preserves homotopy cocartesian cubes. Hence $p^{*} X$ is a homotopy cocartesian $\left.G_{+}\right|_{H^{-c u b e}}$ By the first part of the corollary and $G$-linearity, $\Phi_{*}\left(p^{*} X\right)=p^{*} \Phi_{*}(X)$ is homotopy cartesian. By Proposition A.1, $p^{*}$ detects homotopy cartesian cubes, hence $\Phi_{*}(X)$ is homotopy cartesian.

\section{5 $G$-linearity and adjoint assembly maps}

Let $\mathscr{C}$ and $\mathscr{D}$ be pointed $G$-model categories and let $\Phi: \mathscr{C} \rightarrow \mathscr{D}^{G}$ be an sSet-enriched reduced homotopy functor. Recall from Section 3.2 that, for any finite $G$-set $J$ and any object $c$ of $\mathscr{C}^{G}$, there is a natural adjoint assembly map

$$
\alpha: \Phi(c) \longrightarrow \Omega^{J} \Phi\left(\Sigma^{J} c\right) .
$$

The aim of this section is to explore the relationship between the $G$-linearity of $\Phi$ and the map $\alpha$.

Remark 3.24 Given a cofibrant $G$-object $c$ in $\mathscr{C}^{G}$ and a finite $G$-set $J$, recall the cofibrant $J_{+}$-cube

$$
\left(S^{J} c\right)_{U}= \begin{cases}c & U=\varnothing, \\ C^{U} c & U \lessgtr J_{+}, \\ \Sigma^{J} c & U=J_{+},\end{cases}
$$

from Example 3.4. There is a zig-zag

$$
\Phi(c) \stackrel{\sim}{\longrightarrow} F \Phi(c) \longrightarrow \operatorname{holim}_{\mathcal{P}_{0}\left(J_{+}\right)} F \Phi\left(S^{J} c\right) \stackrel{\sim}{\simeq} F \Phi\left(\Sigma^{J} c\right),
$$

where the last equivalence is induced by the equivalence of fibrant $\mathcal{P}_{0}\left(J_{+}\right)$-diagrams

$$
\left.\omega^{J}\left(F \Phi\left(\Sigma^{J} c\right)\right) \stackrel{\sim}{\longrightarrow} F \Phi\left(S^{J} c\right)\right|_{\mathcal{P}_{0}\left(J_{+}\right)}
$$

for the $G$-diagram $\omega^{J} d$ from Example 1.19 associated to an object $d$ of $\mathscr{D}^{G}$, with vertices $\left(\omega^{J} d\right)_{J_{+}}=d$ and $\left(\omega^{J} d\right)_{U}=*$ for $U \neq J_{+}$. The adjoint assembly map above fits into the commutative diagram

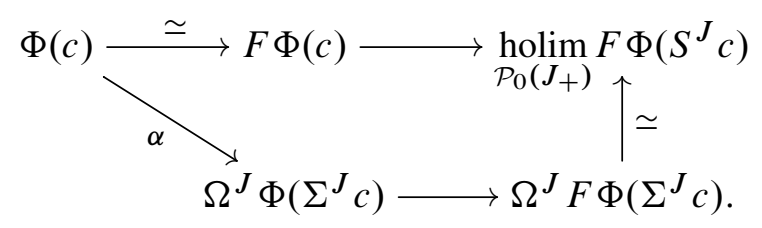


One can think of the map $\Phi(c) \rightarrow \operatorname{holim}_{\mathcal{P}_{0}\left(J_{+}\right)} F \Phi\left(S^{J} c\right)$ as a model for the adjoint assembly map that can be defined also when $\Phi$ is not enriched.

Proposition 3.25 Let $\mathscr{C}$ and $\mathscr{D}$ be pointed $G$-model categories and $\Phi: \mathscr{C} \rightarrow \mathscr{D}^{G}$ an sSet-enriched $G$-linear homotopy functor. For any finite $G$-set $J$ and any cofibrant $G$-object $c \in \mathscr{C}^{G}$, the composite

$$
\Phi(c) \stackrel{\alpha}{\longrightarrow} \Omega^{J} \Phi\left(\Sigma^{J} c\right) \longrightarrow \Omega^{J} F \Phi\left(\Sigma^{J} c\right)
$$

is a weak equivalence in $\mathscr{D}^{G}$.

Proof The decomposition $J_{+} \cong\left(\bigsqcup_{z \in G \backslash J} z\right)_{+}$of $J$ as a disjoint union of transitive $G$-sets gives a factorization the map of the statement as an iterated construction

$$
\Phi(c) \rightarrow \Omega^{z_{1}} F \Phi\left(\Sigma^{z_{1}} c\right) \rightarrow \cdots \rightarrow \Omega^{z_{1}} \cdots \Omega^{z_{n}} F \Phi\left(\Sigma^{z_{1}} \cdots \Sigma^{z_{m}} c\right) .
$$

The functor $\Sigma^{z}(-)$ preserves cocartesian cubes and $\Omega^{z}$ preserves fibrant objects, so, using the natural weak equivalences $\Sigma^{z} \Sigma^{w} c \stackrel{\sim}{\rightarrow} \Sigma^{z \amalg w} d$ for $d$ cofibrant and $\Omega^{z \amalg w} d \stackrel{\sim}{\rightarrow} \Omega^{z} \Omega^{w} d$ for $d$ fibrant, it suffices to show that $\Phi(c) \rightarrow \Omega^{G / H} F \Phi\left(\Sigma^{G / H} c\right)$ is an equivalence for every transitive $G-$ set $G / H$.

By Proposition 3.13, $\Phi$ sends the homotopy cocartesian $G / H_{+}-$cube $S^{G / H_{C}}$ of Remark 3.24 to a homotopy cartesian $G / H_{+}-$cube. That is, the second map in the zig-zag

$$
\Phi(c) \stackrel{\sim}{\longrightarrow} F \Phi(c) \rightarrow \operatorname{holim}_{\mathcal{P}_{0}\left(G / H_{+}\right)} F \Phi\left(S^{G / H} c\right) \stackrel{\sim}{\sim} \Omega^{G / H} F \Phi\left(\Sigma^{G / H} c\right)
$$

is an equivalence in $\mathscr{D}^{G}$. The statement now follows from the commutativity of the diagram in Remark 3.24 above.

We aim at proving a converse to Proposition 3.25. We remind the reader that a simplicial category $\mathscr{C}$ is locally finitely presentable if there is a set $\Theta$ of objects in $\mathscr{C}$ such that every object of $\mathscr{C}$ is isomorphic to a filtered colimit of objects in $\Theta$ and, for every $\theta \in \Theta$, the functor $\operatorname{Map}_{\mathscr{C}}(\theta,-): \mathscr{C} \rightarrow$ sSet preserves filtered colimits (see $[1 ; 17])$. For example, the categories of simplicial sets and of symmetric spectra (of simplicial sets) satisfy this condition.

Theorem 3.26 Let $\mathscr{C}$ and $\mathscr{D}$ be pointed $G$-model categories and suppose that the simplicial categories $\mathscr{D}^{H}$ are locally finitely presentable for every $H \leq G$. Let $\Phi: \mathscr{C} \rightarrow \mathscr{D}^{G}$ be an sSet-enriched reduced homotopy functor and $J$ be a finite $G$-set. If the canonical map

$$
\Phi(c) \longrightarrow \Omega^{\left.J\right|_{H}} F \Phi\left(\Sigma^{\left.J\right|_{H}} c\right)
$$


is a weak equivalence in $\mathscr{D}^{H}$ for every cofibrant object $c \in \mathscr{C}^{H}$ and subgroup $H \leq G$, then the induced functor $\Phi_{*}: \mathscr{C}_{a}^{\mathcal{P}\left(J_{+}\right)} \rightarrow \mathscr{D}_{a}^{\mathcal{P}\left(J_{+}\right)}$sends homotopy cocartesian $\bar{J}_{+}$ cubes to homotopy cartesian $J_{+}-$cubes.

In particular, if $\Phi(c) \stackrel{\sim}{\longrightarrow} \Omega^{\left.G\right|_{H}} F \Phi\left(\left.\Sigma^{G}\right|_{H} c\right)$ is an equivalence for every subgroup $H \leq G$ and cofibrant $H$-object $c$, the functor $\Phi$ is $G$-linear.

The proof of this theorem is technical and it is given at the end of the section.

Remark 3.27 The theorem above holds also in the $G$-model categories of pointed spaces or orthogonal spectra, even though these are not locally finitely presentable. The presentability condition is used to commute a sequential homotopy colimit and a finite equivariant homotopy limit, as explained in Proposition A.8. These commute

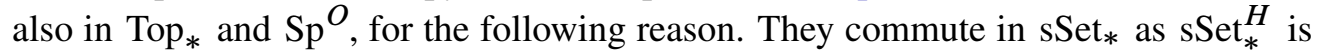
locally finitely presentable. This property can be transported through the $G-$ Quillen equivalence $|-|:$ sSet $_{*} \rightleftarrows \operatorname{Top}_{*}:$ Sing, using that realization commutes with finite limits and Sing with sequential colimits along cofibrations. It can be further deduced for $\mathrm{Sp}^{O}$ as limits and colimits are levelwise.

Corollary 3.28 Under the hypotheses of Theorem 3.26, suppose additionally that the functor $\operatorname{hom}_{H}(G,-): \mathscr{D}^{H} \rightarrow \mathscr{D}^{G}$ detects equivalences of fibrant objects for every subgroup $H$ of $G$. Then the following are equivalent:

(1) $\Phi$ is $G$-linear.

(2) For every cofibrant object $c \in \mathscr{C}^{H}$ and every $H \leq G$, the canonical map $\Phi(c) \rightarrow \Omega^{\left.G\right|_{H}} F \Phi\left(\Sigma^{\left.G\right|_{H}} c\right)$ is an equivalence in $\mathscr{D}^{H}$.

(3) For every finite $G-$ set $J$ the functor $\Phi_{*}: \mathscr{C}_{a}^{\mathcal{P}\left(J_{+}\right)} \rightarrow \mathscr{D}_{a}^{\mathcal{P}\left(J_{+}\right)}$sends homotopy cocartesian $J_{+}$-cubes to homotopy cartesian $J_{+}$-cubes.

Proof $(1) \Longrightarrow$ (2) By Corollary 3.23 the functor $\Phi$ is $H$-linear for every subgroup $H \leq G$. The implication then follows from Proposition 3.25 for the $H$-set $\left.G_{+}\right|_{H}$.

(2) $\Longrightarrow$ (3) By Theorem 3.26 it is enough to show that $\Phi(c) \rightarrow \Omega^{\left.J\right|_{H}} F \Phi\left(\Sigma^{\left.J\right|_{H}} c\right)$ is an equivalence for every finite $G$-set $J$. But $\Phi$ is $G$-linear by Theorem 3.26 and hence $H$-linear by Corollary 3.23. The adjoint assembly is then an equivalence by Proposition 3.25.

(3) $\Longrightarrow$ (1) For $J=G$ the conclusion in (3) is the definition of $G$-linearity. 
Remark 3.29 Define the $G$-differential (at the zero object) of a reduced enriched homotopy functor $\Phi: \mathscr{C} \rightarrow \mathscr{D}^{G}$ to be the functor $D_{*} \Phi: \mathscr{C} \rightarrow \mathscr{D}^{G}$ given by

$$
D_{*} \Phi(c)=\operatorname{hocolim}\left(Q \Phi(c) \rightarrow Q \Omega^{G} F \Phi\left(\Sigma^{G} c\right) \rightarrow Q \Omega^{2 G} F \Phi\left(\Sigma^{2 G} c\right) \rightarrow \cdots\right),
$$

where $n G$ is the disjoint union of $n$ copies of $G$. As a direct consequence of Corollary 3.28(2), the functor $D_{*} \Phi$ is $G$-linear and it is equipped with a universal natural transformation $\Phi \rightarrow D_{*} \Phi$. The argument of [14, Theorem 1.8] applies verbatim to our equivariant situation, showing that $\Phi \rightarrow D_{*} \Phi$ is essentially initial among maps from $\Phi$ to a $G$-excisive functor. This extends [7, Proposition 2.2.5], which assumed an extra "stable excision" condition on $\Phi$.

Proof of Theorem 3.26 We follow the strategy of the proofs of [14, Theorem 1.8 and Lemma $1.9 ; 21]$ of showing that the adjoint assembly map evaluated at a cocartesian cube factors through a cartesian cube. It is convenient to introduce a new model for the loop space. For a cofibrant object $c \in \mathscr{C}^{G}$, we define

$$
\bar{\Omega}^{J} F \Phi\left(\Sigma^{J} c\right):=\operatorname{holim}_{\mathcal{P}_{0}\left(J_{+}\right)} F \Phi\left(S^{J} c\right) .
$$

This object comes with a natural weak equivalence $\bar{\Omega}^{J} F \Phi\left(\Sigma^{J} c\right) \longleftarrow \Omega^{J} F \Phi\left(\Sigma^{J} c\right)$ (see Remark 3.24). Let $X: \mathcal{P}\left(J_{+}\right) \rightarrow \mathscr{C}$ be a cofibrant $J_{+}-$cube. Define a $G$-diagram $K: \mathcal{P}\left(J_{+}\right) \times \mathcal{P}\left(J_{+}\right) \rightarrow \mathscr{C}$ by

$$
K(U, T)=\operatorname{hocolim}_{S \in \mathcal{P}_{1}\left(J_{+}\right)} X_{(S \cap U) \cup T}
$$

and define a $J_{+}$-cube $Y: \mathcal{P}\left(J_{+}\right) \rightarrow \mathscr{D}$ by

$$
Y_{T}=\operatorname{holim}_{\mathcal{P}_{0}\left(J_{+}\right)} F \Phi(K(-, T)) .
$$

The key of this proof is to define, for every $T \subset J_{+}$, a factorization, natural in $T$,

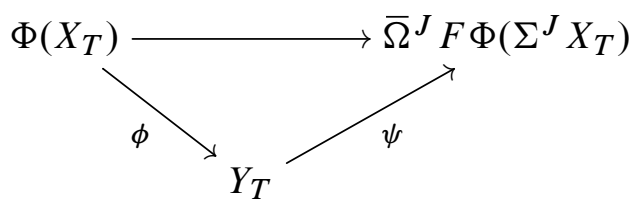

and show that $Y$ is homotopy cartesian when $X$ is homotopy cocartesian. Writing $\Delta^{\widetilde{U}}$ for $N \mathcal{P}_{0}(U)$, the first map of the factorization has $U$-component

$$
\phi_{U}: \Phi\left(X_{T}\right) \longrightarrow \operatorname{map}\left(\Delta^{\widetilde{U}}, F \Phi(K(U, T))\right)
$$

adjoint to the composite

$$
\Delta^{\widetilde{U}} \otimes \Phi\left(X_{T}\right) \rightarrow \Phi\left(\Delta^{\widetilde{U}} \otimes X_{T}\right) \rightarrow \Phi(K(U, T)) \rightarrow F \Phi(K(U, T)),
$$


where the second map is induced by $\Delta^{\widetilde{U}} \otimes X_{T} \rightarrow \Delta^{\widetilde{U}} \otimes X_{T \cup U} \rightarrow K(U, T)$. The map $\psi$ is the homotopy limit over $U$ of the map of diagrams $F \Phi(K(U, T)) \rightarrow F \Phi\left(\left(S^{J} X_{T}\right)_{U}\right)$ induced by the map $K(U, T) \rightarrow\left(S^{J} X_{T}\right)_{U}$ defined as follows. For $U \neq J_{+}$, it is the composite

$$
\begin{aligned}
K(U, T) & =\underset{S \in \mathcal{P}_{1}\left(J_{+}\right)}{\operatorname{hocolim}} X_{(S \cap U) \cup T} \\
& =\underset{S \in \mathcal{P}_{1}\left(J_{+}\right)}{\operatorname{hocolim}}(-\cap U)^{*} X_{S \cup T} \rightarrow \underset{S \in \mathcal{P}(U)}{\operatorname{hocolim}} X_{S \cup T} \rightarrow C^{U} X_{T},
\end{aligned}
$$

where the first arrow is the canonical map induced by $(-\cap U): \mathcal{P}_{1}\left(J_{+}\right) \rightarrow \mathcal{P}(U)$ and the second arrow is induced by collapsing all the non-initial vertices. For $U=J_{+}$, the map is

$$
K\left(J_{+}, T\right)=\underset{S \in \mathcal{P}_{1}\left(J_{+}\right)}{\operatorname{hocolim}} X_{S \cup T} \rightarrow \underset{\mathcal{P}_{1}\left(J_{+}\right)}{\operatorname{hocolim}} \sigma^{J} X_{T}=\Sigma^{J} X_{T}
$$

induced on homotopy colimits by the map of $J_{+}$-cubes given by the identity on the empty set vertex that collapses the other vertices to the point.

Now suppose that $X$ is homotopy cocartesian and let us see that $Y$ is homotopy cartesian. There is a natural equivalence $K(U, T) \stackrel{\sim}{\rightarrow} X_{U \cup T}$. Indeed, the maps $X_{(S \cap U) \cup T} \rightarrow X_{((S \cup\{t\}) \cap U) \cup T}$ are the identity for all $t \in T$, so $K(U, T) \stackrel{\sim}{\rightarrow} X_{U \cup T}$ as long as $T \neq \varnothing$, by Lemma 3.30 below. For $T=\varnothing$ and $U \neq J_{+}$there is a weak equivalence

$$
K(U, \varnothing)=\underset{S \in \mathcal{P}_{1}\left(J_{+}\right)}{\operatorname{hocolim}} X_{S \cap U} \stackrel{\sim}{\longrightarrow} X_{U},
$$

again by Lemma 3.30, as the maps $X_{S \cap U} \rightarrow X_{(S \cup\{v\}) \cap U}$ are the identity for all $v \in J_{+} \backslash U$. Finally,

$$
K\left(J_{+}, \varnothing\right)=\underset{S \in \mathcal{P}_{1}\left(J_{+}\right)}{\operatorname{hocolim}} X_{S} \stackrel{\sim}{\longrightarrow} X_{J_{+}}
$$

since $X$ is assumed to be homotopy cocartesian. This shows that

$$
Y_{T} \stackrel{\sim}{\longrightarrow} \operatorname{holim}_{U \in \mathcal{P}_{0}\left(J_{+}\right)} F \Phi\left(X_{U \cup T}\right) .
$$

For every fixed $U \neq \varnothing$, the cube $T \mapsto F \Phi\left(X_{U \cup T}\right)$ is homotopy cartesian by Lemma 3.30, as the maps $F \Phi\left(X_{U \cup T}\right) \rightarrow F \Phi\left(X_{U \cup T \cup\{u\}}\right)$ are the identity for all $u \in U$. The cube $Y$ is then a homotopy limit of cartesian cubes and therefore also cartesian since homotopy limits commute with each other.

Iterating this construction and using that $\Sigma^{J}$ and $\bar{\Omega}^{J}$ preserve homotopy cocartesian and homotopy cartesian $J_{+}$-cubes, respectively, one gets a factorization of each map in the colimit system

$$
\Phi(X) \stackrel{\sim}{\longrightarrow} \bar{\Omega}^{J} F \Phi\left(\Sigma^{J} X\right) \stackrel{\sim}{\longrightarrow} \bar{\Omega}^{2 J} F \Phi\left(\Sigma^{2 J} X\right) \stackrel{\sim}{\longrightarrow} \ldots
$$


through a homotopy cartesian $J_{+}$-cube $Y^{(n)}$. The maps in this system are weak equivalences, since all maps $\Phi(c) \rightarrow \Omega^{\left.J\right|_{H}} F \Phi\left(\left.\Sigma^{J}\right|_{H} c\right)$ are assumed to be weak equivalences. By (classical) cofinality for diagrams in $\mathscr{D}^{G}$, the homotopy colimit of the sequence above is equivalent to $\operatorname{hocolim}_{n} Y^{(n)}$. By Proposition A.8 we know that, under our presentability assumptions, sequential homotopy colimits preserve homotopy cartesian $J_{+}$-cubes. Therefore $\Phi(X) \simeq \operatorname{hocolim}_{n} Q \bar{\Omega}^{n J} F \Phi\left(\Sigma^{n J} X\right)$ is homotopy cartesian.

Lemma 3.30 Let $J$ be a finite $G$-set, $X: \mathcal{P}(J) \rightarrow \mathscr{C}$ a $J$-cube and $I \subset J$ a nonempty $G$-invariant subset such that the maps $X_{S} \rightarrow X_{S \cup i}$ are isomorphisms for all $S \subset J$ and $i \in I$. If $X$ is a fibrant diagram, it is homotopy cartesian. Similarly, if $X$ is point-wise cofibrant, it is homotopy cocartesian.

Proof Let $\mathcal{P}_{I}(J)$ be the subposet of $\mathcal{P}_{0}(J)$ consisting of non-empty subsets of $J$ that contain $I$ and write $\iota$ for the inclusion map. The map $U \mapsto U \cup I$ defines a retraction $u_{I}: \mathcal{P}_{0}(J) \rightarrow \mathcal{P}_{I}(J)$. The assumption on the maps $X_{S} \rightarrow X_{S \cup i}$ implies that the natural map $X \rightarrow u_{I}^{*} \iota^{*} X$ is an isomorphism. The composite of the maps

$$
\underset{\mathcal{P}_{I}(J)}{\operatorname{holim}} \iota^{*} X \stackrel{\sim}{\longrightarrow} \underset{\mathcal{P}_{0}(J)}{\operatorname{holim}} u_{I}^{*} \iota^{*} X \rightarrow \underset{\mathcal{P}_{I}(J)}{\operatorname{holim}} \iota^{*} u_{I}^{*} \iota^{*} X
$$

is the identity map and the left-hand map is a weak equivalence since $u_{I}$ is right $G$-cofinal. Hence the right-hand map is a weak equivalence. It fits into a commutative diagram

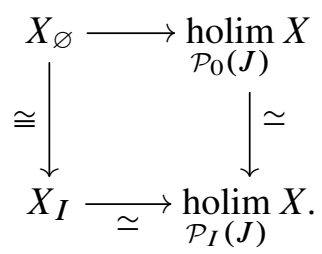

The left vertical map is a $G$-map, which is an isomorphism by assumption, and the bottom horizontal map is a $G$-equivalence since $I$ is initial in $\mathcal{P}_{I}(J)$. Therefore the top map in the square is a weak equivalence and $X$ is homotopy cartesian.

A completely analogous argument shows that $X$ is homotopy cocartesian.

\section{6 $G$-linear functors on pointed $G$-spaces}

Blumberg [4] defines a notion of $G$-linearity for endofunctors of the category of pointed $G$-spaces, for any compact Lie group $G$. When $G$ is finite, we show that his definition and ours agree. 
Before starting, let us remark that when working with spaces we can drop all the point-wise fibrant and cofibrant replacements from the last sections, as homotopy limits and homotopy colimits of $G$-diagrams of spaces are always homotopy invariant. For homotopy limits, this is just because every $G$-space is fibrant. For homotopy colimits, there is a natural homeomorphism

$$
\underset{I}{\operatorname{hocolim} X})^{H} \cong \underset{I^{H}}{\operatorname{hocolim}}\left(\iota_{H}^{*} X\right)^{H}
$$

for every $G$-diagram $X$ in $\left(\operatorname{Top}_{*}\right)_{a}^{I}$ and subgroup $H \leq G$. Here $\iota_{H}: I^{H} \rightarrow I$ is the inclusion of the subcategory of $I$ of objects and morphisms fixed by the $H$-action. Therefore homotopy invariance of homotopy colimits of $G$-diagrams follows from the homotopy invariance of classical homotopy colimits of spaces, proved in [9].

Proposition 3.31 An enriched reduced homotopy functor $\Phi:$ Top $_{*} \rightarrow \operatorname{Top}_{*}^{G}$ is $G-$ linear if and only if the following two conditions hold:

(a) The induced functor $\Phi_{*}:\left(\operatorname{Top}_{*}^{G}\right)^{\mathcal{P}\left(1_{+}\right)} \rightarrow\left(\operatorname{Top}_{*}^{G}\right)^{\mathcal{P}\left(1_{+}\right)}$sends homotopy cocartesian squares of pointed $G$-spaces to homotopy cartesian ones.

(b) For every finite $G$-sets $J$ and every pointed $G$-space $Z$ the natural map

$$
\Phi\left(\bigvee_{J} Z\right) \rightarrow \prod_{J} \Phi(Z)
$$

is an equivalence of pointed $G$-spaces.

Proof If $\Phi$ is $G$-linear, it sends homotopy cocartesian squares to homotopy cartesian squares by Remark 3.14, and the map $\Phi\left(\bigvee_{J} Z\right) \rightarrow \prod_{J} \Phi(Z)$ is an equivalence by Proposition 3.17.

Conversely, Blumberg [4] proves that (a) and (b) imply that the adjoint assembly map $\Phi(Z) \rightarrow \Omega^{V} \Phi\left(Z \wedge S^{V}\right)$ is a $G$-equivalence for every $G$-representation $V$. By Theorem 3.26, this implies the $G$-linearity of $\Phi$.

Remark 3.32 The two conditions of Proposition 3.31 are the definition of $G$-linearity in the case of a finite group $G$ of [4, Hypothesis 3.3] (see also [4, Theorem 3.16]). Blumberg also shows that enriched reduced functors $\Phi:$ Top $_{*} \rightarrow$ Top $_{*}^{G}$ that commute with filtered colimits admit a model structure Quillen equivalent to the stable equivariant category $\left(\mathrm{Sp}^{O}\right)^{G}$, where the fibrant objects are precisely the $G$-linear functors (see [4, Theorem 1.3]). This provides a genuine equivariant analogue of the 1 -homogeneous model structure of [2]. 
Example 3.33 Let $M$ be a commutative well-pointed topological monoid with additive $G$-action and suppose that the fixed point monoids $M^{H}$ are group-like for every subgroup $H$ of $G$. The equivariant Dold-Thom construction $M(-)$ : $\operatorname{Top}_{*} \rightarrow \operatorname{Top}_{*}^{G}$ sends a pointed space $Z$ to the space $M(Z)$ of reduced configurations of points in $Z$ with labels in $M$, with $G$ acting on the labels. After extending $M(-)$ to $\operatorname{Top}_{*}^{G}$, the group acts both on the labels and on the space. If $M$ is discrete, the homotopy groups of $M(-)$ are Bredon cohomology of the Mackey functor $H \mapsto M^{H}$. For a pointed $G$-simplicial set $K$ the simplicial Dold-Thom construction of Example 3.12 compares to the topological one by a natural $G$-homeomorphism $|M(K)| \cong M(|K|)$.

We prove that $M(-)$ : Top ${ }_{*} \rightarrow \mathrm{Top}_{*}^{G}$ is $G$-linear by checking the two conditions from Proposition 3.31. Given a pointed $G$-space $Z$, the fixed points of the map $M(Z) \rightarrow \Omega M\left(Z \wedge S^{1}\right)$ compares by natural homeomorphisms to the adjoint assembly map

$$
M(Z)^{H} \longrightarrow \Omega M\left(Z \wedge S^{1}\right)^{H} \cong \Omega(M(Z))\left(S^{1}\right)^{H} \cong \Omega M(Z)^{H}\left(S^{1}\right)
$$

for the topological group-like monoid $M(Z)^{H}$. This is an equivalence by standard arguments; see [19, Theorem 7.6]. This implies, by Theorem 3.26 for the trivial $G$-set $J=\{1\}$, that the functor $M(-)$ sends homotopy cocartesian squares of $G-$ spaces to homotopy cartesian ones, proving the first property of Proposition 3.31. The second property easily follows, as the map $M\left(\bigvee_{J} Z\right) \rightarrow \prod_{J} M(Z)$ is an equivariant homeomorphism.

Notice that by $G$-linearity the map $M(Z) \rightarrow \Omega^{J} M\left(Z \wedge S^{J}\right)$ is a $G$-equivalence for every finite $G$-set $J$. This shows that the associated Eilenberg-Mac Lane $G$-spectrum $H M_{n}=M\left(S^{n}\right)$ is fibrant in the genuine equivariant stable category $\left(\mathrm{Sp}^{O}\right)^{G}$.

\section{7 $G$-linear functors to $G$-spectra}

We show that the identity functor on $G$-spectra is $G$-linear and deduce from this the classical Wirthmüller isomorphism theorem. We further classify all $G$-linear functors from finite pointed simplicial sets to $G$-spectra.

Let us start by clarifying that, when working with spectra, as for spaces, we can forget all about the point-wise cofibrant and fibrant replacements from the previous sections, thanks to the following result:

Lemma 3.34 Let $G$ be a finite group and $a: G \rightarrow$ Cat be an action of $G$ on a small category $I=a(*)$. Then:

- The homotopy colimit functor hocolim: $\left(\mathrm{Sp}^{O}\right)_{a}^{I} \rightarrow\left(\mathrm{Sp}^{O}\right)^{G}$ preserves weak equivalences between any two diagrams (not necessarily of cofibrant objects). 
- If I has finite-dimensional nerve, holim: $\left(\mathrm{Sp}^{O}\right)_{a}^{I} \rightarrow\left(\mathrm{Sp}^{O}\right)^{G}$ preserves weak equivalences between any two diagrams (not necessarily of fibrant objects).

Proof For any $H$-spectrum $E$ there is a functorial cofibrant replacement $Q E \rightarrow E$, where the map is a level equivalence. By Corollary 2.22 it is enough to show that homotopy colimits preserve level equivalences of maps of $G$-diagrams. Since homotopy colimits of spectra are defined level-wise, this follows from homotopy invariance of homotopy colimits for spaces (see Section 3.6).

For the statement about homotopy limits, take a $G$-diagram of spectra $X$. The nonnegative equivariant homotopy groups of $\operatorname{holim}_{I} X$ are the homotopy groups of the $G$-space

$$
\left.\underset{n}{\operatorname{hocolim}} \Omega^{n G} \underset{I}{(\operatorname{holim}} X\right)(n G) \text {. }
$$

Here we use the notation $E(n G)=E_{n} \wedge O(n) L\left(\mathbb{R}^{n|G|}, n G\right)^{+}$for a $G$-spectrum $E$, where $L\left(\mathbb{R}^{n|G|}, n G\right)$ is the space of isomorphisms of vector spaces from $\mathbb{R}^{n|G|}$ to $n G$. There are natural weak equivalences

$$
\begin{aligned}
\underset{n}{\operatorname{hocolim}} \Omega_{I}^{n G}(\operatorname{holim} X)(n G) & \cong \underset{n}{\operatorname{hocolim}} \Omega_{I}^{n G} \operatorname{holim}(X(n G)) \\
& \cong \underset{n}{\operatorname{hocolim}} \operatorname{holim}_{I} \Omega^{n G}(X(n G)) \\
& \simeq \underset{I}{\longrightarrow} \underset{n}{\operatorname{holim}} \operatorname{hocolim} \Omega^{n G}(X(n G)),
\end{aligned}
$$

where the last map is a weak equivalence by Proposition A.8, as sequential homotopy colimits and finite homotopy limits of $G$-diagrams of spaces commute. Therefore, a weak equivalence of $G$-diagrams of spectra $f: X \rightarrow Y$ induces an isomorphism in non-negative homotopy groups of the homotopy limit precisely when the map $\operatorname{holim}_{I}$ hocolim $_{n} \Omega^{n G} f^{(n G)}$ is an equivalence of $G$-spaces. Since $f$ is an equivalence of $G$-diagrams of spectra, the map hocolim ${ }_{n} \Omega^{n G} f_{i}^{(n G)}$ is an equivalence of $G_{i}$-spaces for all objects $i$ of $I$. It follows by homotopy invariance (Proposition 2.20) that the map of $G$-spaces $\operatorname{holim}_{I}$ hocolim $_{n} \Omega^{n G} f^{(n G)}$ is a weak equivalence since it is a homotopy limit of a weak equivalence of $G$-diagrams of spaces. If $k$ is negative, the equivariant $k^{\text {th }}$ homotopy group of $\operatorname{holim}_{I} X$ is

$\pi_{0}\left(\underset{n}{\operatorname{hocolim}} \Omega^{n G}\left(S h^{-k} \operatorname{holim}_{i \in I} X_{i}\right)(n G)\right)^{G}=\pi_{0}\left(\underset{n}{\operatorname{hocolim}} \Omega^{n G}\left(\operatorname{holim}_{i \in I} S h^{-k} X_{i}\right)(n G)\right)^{G}$,

where, for a $G$-spectrum $E$, the spectrum $S h^{-k} E$ has $n^{\text {th }}$ space $E_{n-k}$. A similar argument shows that, if $f: X \rightarrow Y$ is an equivalence of $G$-diagrams, $\operatorname{holim}_{I} f$ is an equivalence in negative homotopy groups. 
Theorem 3.35 Let $J$ be a finite $G$-set and $a$ be the induced action of $G$ on $\mathcal{P}\left(J_{+}\right)$. Any homotopy cocartesian $J_{+}-$cube $X$ in $\left(\operatorname{Sp}^{O}\right)_{a}^{\mathcal{P}\left(J_{+}\right)}$is homotopy cartesian. That is, the inclusion functor $\mathrm{Sp}^{O} \rightarrow\left(\mathrm{Sp}^{O}\right)^{G}$ is $G$-linear.

In particular, this implies the Wirthmüller isomorphism theorem, stating that for any subgroup $H \leq G$ and $H$-spectrum $E \in\left(\operatorname{Sp}^{O}\right)^{H}$ the canonical map

$$
\eta: G \otimes_{H} E=G_{+} \wedge_{H} E \longrightarrow F_{H}\left(G_{+}, E\right)=\operatorname{hom}_{H}(G, E)
$$

is a weak equivalence of $G$-spectra.

Proof By the equivariant suspension theorem, the map $E \rightarrow \Omega^{\left.G\right|_{H}}\left(E \wedge S^{\left.G\right|_{H}}\right)$ is a weak equivalence for any $H$-spectrum $E$. By Theorem 3.26 (see also Remark 3.27) this is equivalent to $G$-linearity of $\mathrm{Sp}^{O} \rightarrow\left(\mathrm{Sp}^{O}\right)^{G}$. The map $\eta: G \otimes_{H} E \rightarrow \operatorname{hom}_{H}(G, E)$ is a weak equivalence by Theorem 3.20, as $\operatorname{hom}_{H}(G,-):\left(\mathrm{Sp}^{O}\right)^{H} \rightarrow\left(\mathrm{Sp}^{O}\right)^{G}$ preserves weak equivalences.

We end the section with a complete characterization of enriched $G$-linear functors from the category of finite pointed simplicial sets to $G$-spectra.

Proposition 3.36 Let $\Phi: \mathrm{sSet}_{*} \rightarrow\left(\mathrm{Sp}^{O}\right)^{G}$ be an sSet-enriched reduced homotopy functor which commutes with filtered colimits and such that the spectrum $\Phi\left(S^{0}\right)$ is level-wise well-pointed. Then the following conditions are equivalent:

(1) The functor $\Phi$ is $G$-linear.

(2) The functor $\Phi_{*}:\left(\left(\operatorname{sSet}_{*}^{f}\right)^{G}\right)^{\mathcal{P}\left(1_{+}\right)} \rightarrow\left(\left(\mathrm{Sp}^{O}\right)^{G}\right)^{\mathcal{P}\left(1_{+}\right)}$sends homotopy cocartesian squares in $\left(\mathrm{sSet}_{*}^{f}\right)^{G}$ to homotopy cartesian squares of $G$-spectra and $\Phi\left(\bigvee_{J} K\right) \rightarrow \prod_{J} \Phi(K)$ is an equivalence for every finite pointed simplicial $G$-set $K$ and finite $G$-set $J$.

(3) For every finite pointed simplicial $G$-set $K$, the assembly map

$$
\Phi\left(S^{0}\right) \wedge|K| \longrightarrow \Phi(K)
$$

is an equivalence of $G$-spectra.

Proof $(1) \Longrightarrow(2)$ This is true in general, by Remark 3.14 and Proposition 3.17.

(2) $\Longrightarrow$ (3) This can be proven by induction on the skeleton of $K$. The wedges into products condition gives the equivalence for the 0 -skeleton, and the induction step follows from the condition on squares. We refer to [7, Proposition 2.1.4] for the details.

(3) $\Longrightarrow$ (1) Since $\Phi$ commutes with filtered colimits, it is sufficient to show that $\Phi$ is $G$-linear on the full subcategory $\operatorname{sSet}_{*}^{f}$ of finite pointed simplicial sets. By assumption, 
we can equivalently show that $E \wedge|-|$ is $G$-linear on $\operatorname{sSet}_{*}^{f}$ for any level-wise wellpointed $G$-spectrum $E$. If $X: \mathcal{P}\left(G_{+}\right) \rightarrow \operatorname{sSet}_{*}^{f}$ is homotopy cocartesian, the cube of spectra $E \wedge|X|$ is also homotopy cocartesian. Indeed, after applying the geometric fixed points $F^{H}$, the map from the homotopy colimit to the value at $G_{+}$factors as

$$
\begin{aligned}
F^{H}\left(\underset{\mathcal{P}_{1}\left(G_{+}\right)}{\operatorname{hocolim}} E \wedge|X|\right) & \cong F^{H}\left(E \wedge \underset{\mathcal{P}_{1}\left(G_{+}\right)}{\operatorname{hocolim}}|X|\right) \\
& \left.\cong F^{H}(E) \wedge \underset{\mathcal{P}_{1}\left(G_{+}\right)}{\operatorname{hocolim}}|X|\right)^{H} \\
& \simeq F^{H}(E) \wedge\left|X_{G_{+}}\right|^{H} \cong F^{H}\left(E \wedge\left|X_{G_{+}}\right|\right),
\end{aligned}
$$

where the third map is a weak equivalence since $X$ is homotopy cocartesian and smashing with a level-wise well-pointed spectrum preserves weak equivalences. By Theorem 3.35 the diagram $E \wedge X$ is also homotopy cartesian.

Let $\operatorname{Fun}_{\mathrm{Glin}}^{f}\left(\mathrm{sSet}_{*},\left(\mathrm{Sp}^{O}\right)^{G}\right)$ be the category of enriched $G$-linear homotopy functors from pointed simplicial sets to $G$-spectra which commute with filtered colimits.

Corollary 3.37 There is a model structure on the category Fun $_{\mathrm{Glin}^{f}}^{f}\left(\mathrm{sSet}_{*},\left(\mathrm{Sp}^{O}\right)^{G}\right)$ with the equivalences of Definition 3.8 that is Quillen-equivalent to $\left(\mathrm{Sp}^{O}\right)^{G}$.

Proof The evaluation at $S^{0}$ functor $\operatorname{ev}_{S^{0}}$ : $\operatorname{Fun}^{f}\left(\mathrm{sSet}_{*},\left(\mathrm{Sp}^{O}\right)^{G}\right) \rightarrow\left(\mathrm{Sp}^{O}\right)^{G}$ has a left adjoint $L$. It sends a $G$-spectrum $E$ to the functor $L(E)=E \wedge(-): \operatorname{sSet}_{*} \rightarrow\left(\mathrm{Sp}^{O}\right)^{G}$. The unit of the adjunction is the canonical isomorphism $E \cong \mathrm{ev}_{S^{0}}(E \wedge(-))$ and the counit is the assembly map $\operatorname{ev}_{S^{0}}(\Phi) \wedge(-) \rightarrow \Phi$. It is easy to see that $\mathrm{ev}_{S^{0}}$ takes relative $L \mathcal{I}$ (resp $L \mathcal{J}$ ) cell complexes to cofibrations (resp acyclic cofibrations). The argument is analogous to the proof of Theorem 2.6. Hence, by [15, Theorem 11.3.2; 26, Remark D.21], the category $\operatorname{Fun}^{f}\left(\operatorname{sSet}_{*},\left(\operatorname{Sp}^{O}\right)^{G}\right)$ has a model structure where the equivalences and the fibrations are the natural transformations that are sent by $\mathrm{ev}_{S^{0}}$ to equivalences and fibrations in $\left(\mathrm{Sp}^{O}\right)^{G}$, respectively, and

$$
L:\left(\operatorname{Sp}^{O}\right)^{G} \rightleftarrows \operatorname{Fun}^{f}\left(\operatorname{sSet}_{*},\left(\operatorname{Sp}^{O}\right)^{G}\right): \operatorname{ev}_{S^{O}}
$$

is a Quillen adjunction. A natural transformation map $L E \rightarrow \Phi$ is an equivalence, by definition, if and only if $E \rightarrow \Phi\left(S^{0}\right)=e v_{S^{0}} \Phi$ is an equivalence. Therefore $\left(L, \mathrm{ev}_{S^{0}}\right)$ is a Quillen equivalence. By Proposition 3.36, a natural transformation $\Lambda: \Phi \rightarrow \Phi^{\prime}$ is an equivalence at $S^{0}$ if and only if it is an equivalence on finite pointed simplicial $H$-sets for every $H \leq G$, since $\Phi$ and $\Phi^{\prime}$ commute with filtered colimits on all simplicial $H-$ sets. 


\section{Appendix}

\section{A.1 Computing homotopy (co)limits of punctured cubes}

We compare homotopy limits and colimits of punctured cubes of different sizes, specifically how functors between categories of cubes in $\mathscr{C}$ induced by maps $p: K \rightarrow J$ of finite $G$-sets behave on homotopy cartesian and cocartesian cubes.

Proposition A.1 Let $p: K \rightarrow J$ be a surjective equivariant map of finite $G$-sets. Taking the image by $p$ induces an equivariant functor $p_{0}: \mathcal{P}_{0}(K) \rightarrow \mathcal{P}_{0}(J)$, which is left $G$-cofinal. In particular, the induced functor $p^{*}: \mathscr{C}_{a}^{\mathcal{P}(J)} \rightarrow \mathscr{C}_{a}^{\mathcal{P}(K)}$ preserves and detects homotopy cartesian cubes.

Proof We show that, for any subgroup $H \leq G$ and any non-empty object $U \in \mathcal{P}(J)^{H}$, the set $p^{-1}(U) \subset K$ is the final object of $\left(p_{0} / U\right)^{H}$. It is non-empty since $p$ is assumed to be surjective, and clearly satisfies $p p^{-1}(U)=U \subset U$. It is final since objects $V \in\left(p_{0} / U\right)^{H}$ satisfy $p(V) \subset U$, therefore

$$
V \subset p^{-1} p(V) \subset p^{-1}(U) .
$$

This shows that $p_{0}$ is left $G$-cofinal. Now let $X: \mathcal{P}(J) \rightarrow \mathscr{C}$ be a $J$-cube and $X \stackrel{\sim}{\longrightarrow} F X$ a fibrant replacement. There is a commutative diagram

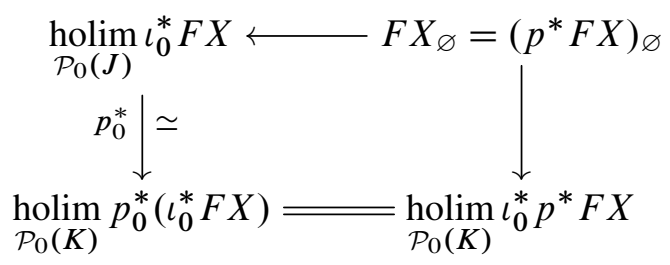

where the left vertical map is an equivalence by $G$-cofinality (Theorem 2.25). Here, $\iota_{0}: \mathcal{P}_{0}(J) \rightarrow \mathcal{P}(J)$ is the inclusion. Notice moreover that $p^{*} X \stackrel{\sim}{\longrightarrow} p^{*} F X$ is a fibrant replacement for $p^{*} X$, as for every subset $S \subset K$ there is an inclusion of the stabilizer groups $G_{S} \leq G_{p(S)}$ and the forgetful functor $\mathscr{C}^{G_{p(S)}} \rightarrow \mathscr{C}^{G_{S}}$ preserves fibrant objects and equivalences by assumption. From the diagram above we see that $X$ is homotopy cartesian if and only if $p^{*} X$ is.

Looking for a similar statement for the behavior of $p^{*}$ on cocartesian cubes, we run into the problem that $p$ does not restrict to a functor $\mathcal{P}_{1}(K) \rightarrow \mathcal{P}_{1}(J)$. There is a formally dual version of the proof of Proposition A.1 that uses the complement dualities on $\mathcal{P}(K)$ and $\mathcal{P}(J)$, but it involves a functor $\mathscr{C}_{a}^{\mathcal{P}(J)} \rightarrow \mathscr{C}_{a}^{\mathcal{P}(K)}$ different from $p^{*}$. This is discussed in Proposition A.5 below. In order to understand the interaction between 
$p^{*}$ and cocartesian cubes we need to introduce a new functor. Let $p^{-1}(j) \subset K$ denote the fiber of an element $j \in J$ and consider the equivariant functor

$$
\lambda:\left(\prod_{j \in J} \mathcal{P}_{1}\left(p^{-1}(j)\right)\right) \times \mathcal{P}_{1}(J) \rightarrow \mathcal{P}_{1}(K)
$$

that sends $\left(\left\{U_{j}\right\}_{j \in J}, V\right)$ to $\left(\bigsqcup_{j \in J} U_{j}\right) \cup p^{-1}(V)$. The product $\prod_{j \in J} \mathcal{P}_{1}\left(p^{-1}(j)\right)$ is the limit of the $G$-diagram of categories $j \mapsto \mathcal{P}_{1}\left(p^{-1}(j)\right)$ with the $G$-structure induced by the $G$-action on $J$.

The functor $\lambda$ is a categorical analogue of a homeomorphism

$$
\left(\prod_{j \in J} \Delta^{\left|p^{-1}(j)\right|-1}\right) \times \Delta^{|J|-1} \cong \Delta^{|K|-1} .
$$

Example A.2 - If $p: K_{+} \rightarrow 1_{+}$is the pointed map that sends all the elements of $K$ to 1 , the product of the fibers is simply $\mathcal{P}_{1}(K)$ and the functor

$$
\lambda: \mathcal{P}_{1}(K) \times \mathcal{P}_{1}\left(1_{+}\right) \rightarrow \mathcal{P}_{1}\left(K_{+}\right)
$$

is analogous to a homeomorphism $\Delta^{\widetilde{K}} \times \Delta^{1} \cong \Delta^{K}$ that splits off a copy of the trivial representation from the permutation representation of $K$. This is written in a more familiar form as $\overline{\mathbb{R}}[K] \times \mathbb{R} \cong \mathbb{R}[K]$. One could think of the product of the categories $\mathcal{P}_{1}\left(p^{-1}(j)\right)$ as an orthogonal complement for the image of the embedding $p^{-1}(-): \mathcal{P}_{1}(J) \rightarrow \mathcal{P}_{1}(K)$.

- Let $I$ and $J$ be finite $G$-sets and consider the pointed projection $p:(I \amalg J)_{+} \rightarrow J_{+}$ that sends $J$ to $J$ by the identity and $I$ to the basepoint + . The preimages over the elements of $J$ consist of a single point and the preimage over the basepoint is $p^{-1}(+)=I_{+}$. The functor $\lambda$ above is the functor

$$
\lambda: \mathcal{P}_{1}\left(I_{+}\right) \times \mathcal{P}_{1}\left(J_{+}\right) \longrightarrow \mathcal{P}_{1}\left((I \amalg J)_{+}\right)
$$

that sends $(U, V)$ to $U \cup V$. It is analogous to the standard homeomorphism of permutation representations $\mathbb{R}[I] \times \mathbb{R}[J] \cong \mathbb{R}[I \amalg J]$.

Proposition A.3 For a surjective equivariant map $p: K \rightarrow J$, the functor $\lambda$ above is right $G$-cofinal. Moreover, the functor $p^{*}: \mathscr{C}_{a}^{\mathcal{P}(J)} \rightarrow \mathscr{C}_{a}^{\mathcal{P}(K)}$ preserves homotopy cocartesian cubes.

Proof Let us first prove that $\lambda$ is well-defined, that is, it does not take the value $K$. Write for simplicity $\underline{U}=\left\{U_{j}\right\}_{j \in J}$ and $\bigsqcup \underline{U}=\bigsqcup_{j \in J} U_{j}$. Suppose that $\lambda(\underline{U}, V)=$ $(\amalg \underline{U}) \cup p^{-1}(V)=K$. Take $j$ in the complement of $V$ in $J$. The fiber $p^{-1}(j) \subset K$ 
is disjoint from $p^{-1}(V)$, but it is covered by the collection $\underline{U}$. As each $U_{i}$ is contained in $p^{-1}(i)$ we must have $U_{j}=p^{-1}(j)$, but this is absurd since $U_{j}$ is a proper subset of $p^{-1}(j)$.

Now let $W$ be an $H$-invariant proper subset of $K$. We show that the right fiber category $W / \lambda$ is $H$-contractible by defining a zig-zag of natural transformations between the identity functor and the projection onto the $H$-invariant object $\left(\varnothing=\{\varnothing\}_{j \in J}, p(W)\right)$ of $W / \lambda$. This is well-defined as $\lambda\left(\{\varnothing\}_{j \in J}, p(W)\right)=p^{-1} p(W)$, which contains $W$. The intermediate functor of the zig-zag is the equivariant functor $\tau: W / \lambda \rightarrow W / \lambda$ defined by

$$
\tau(\underline{U}, V)=(\underline{U}, p(\coprod \underline{U}) \cup V) .
$$

The values of $\tau$ are indeed objects of $W / \lambda$, since $\lambda(\tau(\underline{U}, V))$ clearly contains $(\bigsqcup \underline{U}) \cup p^{-1}(V)$, which in turn contains $W$ as $(\underline{U}, V)$ belongs to $W / \lambda$. There is a zig-zag of natural transformations

$$
\mathrm{id} \longrightarrow \tau \longleftarrow(\underline{\varnothing}, p(W)) .
$$

Both maps are obvious on the first component. The second component of the rightward pointing map is the inclusion $V \subset p(\amalg \underline{U}) \cup V$. The second component of the leftpointing map is induced by the inclusion $W \subset \lambda(\underline{U}, V)$, which when projected down to $J$ gives $p(W) \subset p(\amalg \underline{U}) \cup p p^{-1}(V)=p(\amalg \underline{U}) \cup V$. The zig-zag above realizes to a contracting $H$-invariant homotopy of the category $W / \lambda$, showing that $\lambda$ is right $G$-cofinal.

Now let $X \in \mathscr{C}_{a}^{\mathcal{P}(J)}$ be a cocartesian $J$-cube and $Q X \stackrel{\sim}{\longrightarrow} X$ a point-wise cofibrant replacement. As in the proof of Proposition A.1, notice that $p^{*} Q X \stackrel{\sim}{\longrightarrow} p^{*} X$ is a point-wise cofibrant replacement of $p^{*} X$. Let us compute the homotopy colimit of $p^{*} Q X$ over $\mathcal{P}_{1}(K)$. By $G$-cofinality and Corollary 2.26 , there are $G$-equivalences

$$
\begin{aligned}
\underset{\mathcal{P}_{1}(K)}{\operatorname{hocolim}} p^{*} Q X & \simeq \underset{\left(\prod_{j \in J} \mathcal{P}_{1}\left(p^{-1}(j)\right)\right) \times \mathcal{P}_{1}(J)}{\operatorname{hocolim}} \lambda^{*} p^{*} Q X \\
& \simeq \underset{\left(\prod_{j \in J} \mathcal{P}_{1}\left(p^{-1}(j)\right)\right)}{\operatorname{hocolim}} \underset{\mathcal{P}_{1}(J)}{\operatorname{hocolim}} \lambda^{*} p^{*} Q X .
\end{aligned}
$$

We claim that, for every fixed collection $\underline{U}$ of subsets of the fibers, the canonical map

$$
\phi_{\underline{U}}: \underset{\mathcal{P}_{1}(J)}{\operatorname{hocolim}}\left(\lambda^{*} p^{*} Q X\right)_{(\underline{U},-)} \rightarrow X_{J}
$$

is a $G_{\underline{U}}$-equivalence. From this claim it follows by homotopy invariance of the homotopy colimit that hocolim $\mathcal{P}_{\mathcal{P}_{1}(K)} p^{*} Q X$ is equivalent to the homotopy colimit over $\prod_{j} \mathcal{P}_{1}\left(p^{-1}(j)\right)$ of the constant $G$-diagram with value $X_{J}$. Since the indexing category is $G$-contractible (it has a $G$-invariant initial object) this is $G$-equivalent to 
$X_{J}=\left(p^{*} X\right)_{K}$, proving that $p^{*} Q X$ is homotopy cocartesian. Let us finally show that $\phi_{\underline{U}}$ is a weak equivalence. The diagram

$$
Z^{\underline{U}}:=\left(\lambda^{*} p^{*} Q X\right)_{(\underline{U},-)}=Q X_{p(\amalg \underline{U}) \cup(-)}
$$

is a $J$-cube with the $G$-action on $J$ restricted to the stabilizer group $G_{\underline{U}}$. Then $\phi_{\underline{U}}$ is an equivalence precisely when $Z \underline{U}$ is homotopy cocartesian. If any of the sets $\bar{U}_{j}$ is non-empty, the maps $\left(Z^{\underline{U}}\right)_{V} \rightarrow\left(Z^{\underline{U}}\right)_{V \cup j}$ are identities for every subset $V \subset J$. We proved in Lemma 3.30 that in this case $Z^{\underline{U}}$ is homotopy cocartesian. For the family of empty sets $\underline{U}=\varnothing$, the $J$-cube $Z \underline{\varnothing}$ is the cube $X$, which is assumed to be homotopy cocartesian.

Remark A.4 In general $p^{*}: \mathscr{C}_{a}^{\mathcal{P}(J)} \rightarrow \mathscr{C}_{a}^{\mathcal{P}(K)}$ does not detect homotopy cocartesian cubes. In the proof of Proposition A.3 we constructed an equivalence over $X_{J}$,

$$
\underset{\mathcal{P}_{1}(K)}{\operatorname{hocolim}} p^{*} Q X \simeq \underset{\prod_{j \in J} \mathcal{P}_{1}\left(p^{-1}(j)\right)}{\operatorname{hocolim}} Y,
$$

where $Y$ is the diagram that sends $\varnothing=(\varnothing, \ldots, \varnothing)$ to hocolim $\mathcal{P}_{\mathcal{P}_{1}(J)} Q X$ and all the other vertices to $X_{J}$. If $p^{*} X$ is homotopy cocartesian, the left-hand side is also equivalent to $X_{J}$, but this is in general not enough to conclude that $Y_{\varnothing}$ is equivalent to $X_{J}$. However, this is the case if $\mathscr{C}$ is the category of spectra, as homotopy cocartesian $J$-cubes are the same as homotopy cartesian $J$-cubes (see Theorem 3.35). Hence the functor $p^{*}:\left(\mathrm{Sp}^{O}\right)_{a}^{\mathcal{P}(J)} \rightarrow\left(\mathrm{Sp}^{O}\right)_{a}^{\mathcal{P}(K)}$ preserves and detects homotopy cocartesian cubes.

We end this section by discussing the duals of Propositions A.1 and A.3. For an equivariant surjective map of finite $G$-sets $p: K \rightarrow J$, let $\bar{p}: \mathcal{P}(K) \rightarrow \mathcal{P}(J)$ be the composite functor

$$
\bar{p}: \mathcal{P}(K) \longrightarrow \mathcal{P}(K)^{\mathrm{op}} \stackrel{p^{\mathrm{op}}}{\longrightarrow} \mathcal{P}(J)^{\mathrm{op}} \longrightarrow \mathcal{P}(J)
$$

that sends a subset $U$ of $K$ to $J \backslash p(K \backslash U)$. The dual of the functor $\lambda$ is defined by a similar composition, and an easy calculation shows that it is the functor

$$
\bar{\lambda}:\left(\prod_{j \in J} \mathcal{P}_{0}\left(p^{-1}(j)\right)\right) \times \mathcal{P}_{0}(J) \rightarrow \mathcal{P}_{0}(K)
$$

that sends $(\underline{U}, V)$ to $(\amalg \underline{U}) \cap p^{-1}(V)$. The dual proofs of Propositions A.1 and A.3 give the following:

Proposition A.5 The restriction $\bar{p}: \mathcal{P}_{1}(K) \rightarrow \mathcal{P}_{1}(J)$ is right $G$-cofinal and the functor $\bar{\lambda}$ is left $G$-cofinal. It follows that $\bar{p}^{*}: \mathscr{C}_{a}^{\mathcal{P}(J)} \rightarrow \mathscr{C}_{a}^{\mathcal{P}(K)}$ preserves and detects homotopy cocartesian cubes and preserves homotopy cartesian cubes. 
We end by noticing that this picture does not have an analogue for injective equivariant maps $\iota: J \rightarrow K$. It is easy to see that restricting along $\iota$ does not preserve any cartesian or cocartesian properties of cubes. The right thing to study seems to be the preimage functor $\iota^{-1}: \mathcal{P}(K) \rightarrow \mathcal{P}(J)$, but this does not restrict to either $\mathcal{P}_{0}(K) \rightarrow \mathcal{P}_{0}(J)$ or $\mathcal{P}_{1}(K) \rightarrow \mathcal{P}_{1}(J)$. However, if $J$ and $K$ are pointed and $\iota$ preserves the basepoint, there is a retraction $p: K \rightarrow J$ that collapses the complement of the image of $\iota$ onto the basepoint. In this case we can simply consider $p^{*}$.

\section{A.2 Finite categories and cofibrant $G$-diagrams}

We give a criterion for determining if a $G$-diagram is cofibrant in the model structure of Theorem 2.6 when the over-categories of the indexing category $I$ have finitedimensional nerve. Such categories are sometimes called directed Reedy categories. The criterion is in terms of latching maps and it is completely analogous to the classical theory (see eg [15, Section 15]).

Let $\mathscr{C}$ be a cocomplete category. We denote by $(I / i)^{\prime}$ the over-category $I / i$ with the object $i=i$ removed. The latching diagram of a diagram $X: I \rightarrow \mathscr{C}$ is the diagram $L(X): I \rightarrow \mathscr{C}$ given on objects by

$$
L(X)_{i}=\operatorname{colim}\left((I / i)^{\prime} \longrightarrow I \stackrel{X}{\longrightarrow} \mathscr{C}\right)
$$

and on morphisms $f: i \rightarrow j$ by the map induced on colimits by $f_{*}:(I / i)^{\prime} \rightarrow(I / j)^{\prime}$. The inclusions $(I / i)^{\prime} \hookrightarrow I / i$ induce maps $L(X)_{i} \rightarrow \operatorname{colim}_{I / i} u_{i}^{*} X \cong X_{i}$, which give a natural transformation $L(X) \rightarrow X$.

If $X \in \mathscr{C}_{a}^{I}$ is a $G$-diagram, the latching diagram $L(X)$ inherits a $G$-structure. The structure maps are the composite maps

$$
L(X)_{i} \stackrel{L\left(g_{X}\right)}{\longrightarrow} \operatorname{colim}\left((I / i)^{\prime} \stackrel{g}{\longrightarrow}(I / g i)^{\prime} \longrightarrow I \stackrel{X}{\longrightarrow} \mathscr{C}\right) \longrightarrow L(X)_{g i}
$$

induced by taking colimits of the compositions in the diagram

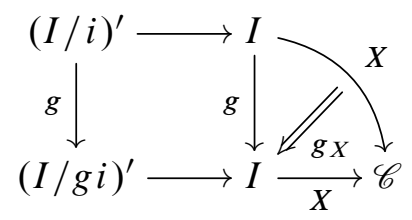

and the canonical map $L(X) \rightarrow X$ is a map of $G$-diagrams.

Proposition A.6 Let $\mathscr{C}$ be a $G$-model category (see Definition 2.1), and I a category with $G$-action such that the simplicial set $N I / i$ is finite-dimensional for every object $i$ in $I$. Let $X$ be an object of $\mathscr{C}_{a}^{I}$ such that for every object $i$ in $I$ the map $L(X)_{i} \rightarrow X_{i}$ is a cofibration in $\mathscr{C}^{G_{i}}$. Then $X$ is cofibrant in the model structure on $\mathscr{C}_{a}^{I}$ of Theorem 2.6. 
Proof In order to show that $X$ is cofibrant we need to define a lift for every diagram in $\mathscr{C}_{a}^{I}$

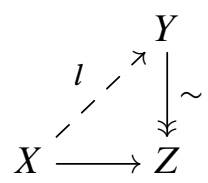

where the vertical map is an acyclic fibration. We build this lift by induction on a filtration of $I$ defined by the degree function $\operatorname{deg}:$ ob $I \rightarrow \mathbb{N}$,

$$
\operatorname{deg}(i)=\operatorname{dim} N I / i
$$

It is easy to see that the degree function is equivariant (where $\mathbb{N}$ has trivial action) and that if $\alpha: i \rightarrow j$ is a non-identity morphism then $\operatorname{deg}(i)<\operatorname{deg}(j)$. Let $I_{\leq n}$ be the full subcategory of $I$ with objects of degree less than or equal to $n$. Since the degree function is equivariant, the $G$-action of $I$ restricts to $I_{\leq n}$ and the $G$-structure on $X$ restricts to a $G$-structure on the restricted diagram $X_{\leq n}: I_{\leq n} \rightarrow I \stackrel{X}{\longrightarrow} \mathscr{C}$. We build the lift inductively on the diagrams $X_{\leq n}$.

For the base step, choose a section $s:$ ob $I_{\leq 0} / G \rightarrow$ ob $I_{\leq 0}$. For each orbit $\gamma \in \mathrm{ob} I_{\leq 0} / G$ one can choose a $G_{s(\gamma)}$-equivariant lift

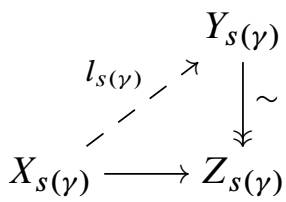

since the map $\varnothing=L(X)_{s(\gamma)} \rightarrow X_{S(\gamma)}$ is a cofibration in $\mathscr{C}^{G_{s(\gamma)}}$ by assumption (the map $Y_{s(\gamma)} \rightarrow Z_{s(\gamma)}$ is an acyclic fibration of $\mathscr{C}^{\boldsymbol{G}_{s(\gamma)}}$ as equivalences and fibrations in $\mathscr{C}_{a}^{I}$ are point-wise). Given any object $i \in I_{\leq 0}$ outside the image of $s$, define $l_{i}: X_{i} \rightarrow Y_{i}$ as the composite

$$
X_{i} \stackrel{g^{-1}}{\longrightarrow} X_{S([i])} \stackrel{l_{s[i]}}{\longrightarrow} Y_{S([i])} \stackrel{g}{\longrightarrow} Y_{i}
$$

for a choice of $g \in G$ with $g s[i]=i$. Since the category $I_{\leq 0}$ is discrete (a $G$-set) by the properties of the degree function, these lifts define a map of diagrams $l^{0}: X_{\leq 0} \rightarrow Y_{\leq 0}$ lifting $X_{\leq 0} \rightarrow Z_{\leq 0}$. Moreover, $l$ respects the $G$-structure since the lifts $l_{s(\gamma)}$ are $G_{s(\gamma)}$-equivariant.

Now suppose we defined a lift $l^{n-1}: X_{\leq n-1} \rightarrow Y_{\leq n-1}$. Let $I_{n}$ be the full subcategory of $I$ with objects of degree $n$. Choose a section $s^{n}:$ ob $I_{n} / G \rightarrow$ ob $I_{n}$ and, for every 
$\gamma \in$ ob $I_{n} / G$, a lift in $\mathscr{C}^{G_{s}^{n}(\gamma)}:$

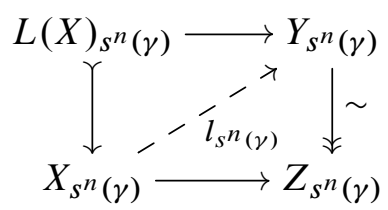

The top horizontal map is the canonical map given by the universal property of the colimits defining $L(X)$. Again, the lifts exist because $L(X)_{S^{n}(\gamma)} \rightarrow X_{S^{n}(\gamma)}$ is a cofibration. For a general object $i$ of $I_{n}$ define

$$
l_{i}: X_{i} \stackrel{g^{-1}}{\longrightarrow} X_{S([i])} \stackrel{l_{s[i]}}{\longrightarrow} Y_{s([i])} \stackrel{g}{\longrightarrow} Y_{i} .
$$

Commutativity of the diagram above ensures that the resulting map $l^{n}: X_{\leq n} \rightarrow Y_{\leq n}$ commutes with the structure maps of $X_{\leq n}$ and $Y_{\leq n}$. Moreover $l^{n}$ respects the $G-$ structure by $G_{s^{n}(\gamma)}$-equivariance of $l_{s(\gamma)}$.

\section{A.3 Sequential homotopy colimits and finite $G$-homotopy limits}

Definition A.7 [17] A simplicial category $\mathscr{C}$ is locally finitely presentable if there is a set of objects $\Theta$ satisfying

(1) for every $c \in \Theta$ the mapping space functor

$$
\operatorname{Map}_{\mathscr{C}}(c,-): \mathscr{C} \longrightarrow \mathrm{sSet}
$$

preserves filtered colimits,

(2) every object of $\mathscr{C}$ is isomorphic to a filtered colimit of objects in $\Theta$.

When $\mathscr{C}$ is locally finitely presented the functor $\operatorname{map}_{\mathscr{C}}(K,-)$ commutes with filtered colimits if $K$ is a finite simplicial set. This follows from the conditions above and an adjunction argument. We consider the poset category $\mathbb{N}$ of natural numbers as a category with trivial $G$-action.

Proposition A.8 Let $\mathscr{C}$ be a $G$-model category and suppose that the underlying simplicial categories $\mathscr{C}^{H}$ are locally finitely presentable for all $H \leq G$. Let $J$ be a finite $G$-set and $X: \mathbb{N} \times \mathcal{P}\left(J_{+}\right) \rightarrow \mathscr{C}$ a $G$-diagram with the property that, for every $n \in \mathbb{N}$, the $J_{+}-$cube $X_{n}$ is homotopy cartesian. Then the $J_{+}-$cube hocolim $\mathbb{N} Q X_{n}$ is also homotopy cartesian. 
Proof We must show that the top horizontal map in the commutative diagram

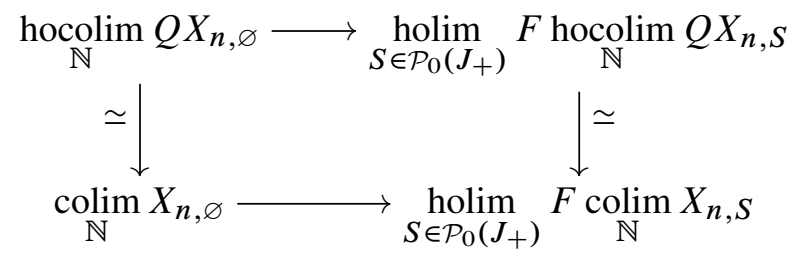

is a weak equivalence in $\mathscr{C}^{G}$. The left-hand vertical map is an equivalence since, in the locally finitely presentable category, $\mathscr{C}^{G}$ filtered colimits are homotopy invariant (see eg [8, Proposition 7.3], or [5] for simplicial sets). Similarly, the right-hand vertical map is the homotopy limit of an equivalence of point-wise fibrant $G$-diagrams, as each $\mathscr{C}^{G_{S}}$ is locally finitely presentable. The bottom map can be factored as

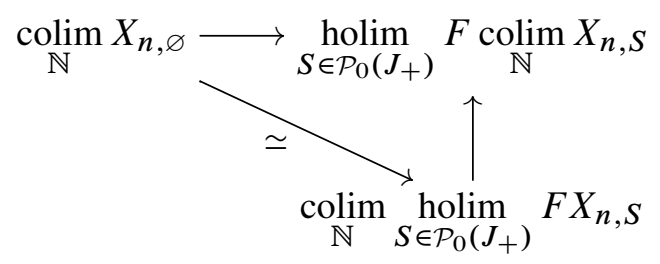

with the diagonal map an equivalence in $\mathscr{C}^{G}$ since $X_{n}$ is homotopy cartesian and filtered colimits in $\mathscr{C}^{G}$ preserve equivalences. To show that the vertical map is an equivalence, we compute from the definition of homotopy limits. Letting

$$
K_{S}=N \mathcal{P}_{0}(S)
$$

we have isomorphisms in $\mathscr{C}^{G}$

$\underset{\mathbb{N}}{\operatorname{colim}} \operatorname{holim}_{S \in \mathcal{P}_{0}\left(J_{+}\right)} F X_{n, S}$

$$
\begin{aligned}
& =\underset{\mathbb{N}}{\operatorname{colim} \lim }\left(\prod_{S} \operatorname{map}_{\mathscr{C}}\left(K_{S}, F X_{n, S}\right) \rightrightarrows \prod_{S \rightarrow T} \operatorname{map}_{\mathscr{C}}\left(K_{S}, F X_{n, T}\right)\right) \\
& \cong \lim \left(\prod_{S} \operatorname{map}_{\mathscr{C}}\left(K_{S}, \underset{\mathbb{N}}{\operatorname{colim}} F X_{n, S}\right) \rightrightarrows \prod_{S \rightarrow T} \operatorname{map}_{\mathscr{C}}\left(K_{S}, \underset{\mathbb{N}}{\operatorname{colim}} F X_{n, T}\right)\right) \\
& =\underset{S \in \mathcal{P}_{0}\left(J_{+}\right)}{\operatorname{holim}} \operatorname{colim} F X_{n, S},
\end{aligned}
$$

where the middle map is an isomorphism because sequential colimits commute with finite limits and with the functors $\operatorname{map}_{\mathscr{C}}\left(K_{S},-\right)$, since each $K_{S}$ is finite. Now let

$$
\overline{F X} \stackrel{\sim}{\rightarrow} F X
$$


be a replacement of $F X$ by a sequence of diagrams such that for each $S \subset J_{+}$the sequence $\overline{F X}_{S}$ is a sequence of $G_{S}$-cofibrations. There is a commutative diagram $\underset{\mathbb{N}}{\operatorname{colim}} \operatorname{holim}_{S \in \mathcal{P}_{0}\left(J_{+}\right)} F X_{n, S} \cong \operatorname{holim}_{S \in \mathcal{P}_{0}\left(J_{+}\right)} \operatorname{colim}_{\mathbb{N}} F X_{n, S} \stackrel{\sim}{\underset{S \in \mathcal{P}_{0}\left(J_{+}\right)}{\operatorname{holim}}} \underset{\mathbb{N}}{\operatorname{colim}} \overline{F X}_{n, S}$

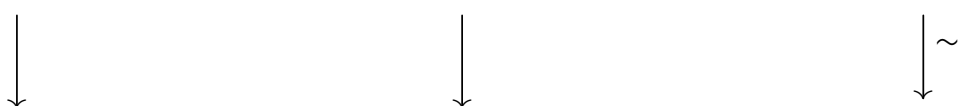

$\operatorname{holim}_{S \in \mathcal{P}_{0}\left(J_{+}\right)} F \underset{\mathbb{N}}{\operatorname{colim}} X_{n, S} \stackrel{\sim}{\longrightarrow} \operatorname{holim}_{S \in \mathcal{P}_{0}\left(J_{+}\right)} F \underset{\mathbb{N}}{\operatorname{colim}} F X_{n, S} \stackrel{\sim}{\longleftarrow} \operatorname{holim}_{S \in \mathcal{P}_{0}\left(J_{+}\right)} F \underset{\mathbb{N}}{\operatorname{colim}} \overline{F X_{n}}, S$

where the right-hand vertical is a weak equivalence because colim ${ }_{\mathbb{N}} \overline{F X}_{n, S}$ is fibrant by an application of the small object argument in the cofibrantly generated model category $\mathscr{C}^{G}$ (see eg [23, Lemma 1.3.2]). It follows that the left-hand vertical map is a weak equivalence, as desired.

\section{References}

[1] J Adámek, J Rosický, Locally presentable and accessible categories, London Mathematical Society Lecture Note Series 189, Cambridge Univ. Press (1994) MR1294136

[2] G Biedermann, B Chorny, O Röndigs, Calculus of functors and model categories, Adv. Math. 214 (2007) 92-115 MR2348024

[3] G Biedermann, O Röndigs, Calculus of functors and model categories, II, Algebr. Geom. Topol. 14 (2014) 2853-2913 MR3276850

[4] A J Blumberg, Continuous functors as a model for the equivariant stable homotopy category, Algebr. Geom. Topol. 6 (2006) 2257-2295 MR2286026

[5] A K Bousfield, D M Kan, Homotopy limits, completions and localizations, Lecture Notes in Mathematics 304, Springer, Berlin (1972) MR0365573

[6] W Chachólski, J Scherer, Homotopy theory of diagrams, Mem. Amer. Math. Soc. 736, Amer. Math. Soc. (2002) MR1879153

[7] E Dotto, Equivariant calculus of functors and $\mathbb{Z} / 2$-analyticity of real algebraic $K$ theory, J. Inst. Math. Jussieu (2015) Online publication

[8] D Dugger, Combinatorial model categories have presentations, Adv. Math. 164 (2001) 177-201 MR1870516

[9] D Dugger, D C Isaksen, Topological hypercovers and $\mathbb{A}^{1}$-realizations, Math. Z. 246 (2004) 667-689 MR2045835

[10] D Dugger, B Shipley, Enriched model categories and an application to additive endomorphism spectra, Theory Appl. Categ. 18 (2007) 400-439 MR2342167

[11] A D Elmendorf, Systems of fixed point sets, Trans. Amer. Math. Soc. 277 (1983) 275-284 MR690052 
[12] P G Goerss, J F Jardine, Simplicial homotopy theory, Progress in Mathematics 174, Birkhäuser, Basel (1999) MR1711612

[13] T G Goodwillie, Calculus, II: Analytic functors, K-Theory 5 (1991/92) 295-332 MR1162445

[14] T G Goodwillie, Calculus, III: Taylor series, Geom. Topol. 7 (2003) 645-711 MR2026544

[15] PS Hirschhorn, Model categories and their localizations, Mathematical Surveys and Monographs 99, Amer. Math. Soc. (2003) MR1944041

[16] S Jackowski, J Słomińska, G-functors, $G$-posets and homotopy decompositions of G-spaces, Fund. Math. 169 (2001) 249-287 MR1852128

[17] G M Kelly, Structures defined by finite limits in the enriched context, I, Cahiers Topologie Géom. Différentielle 23 (1982) 3-42 MR648793

[18] M A Mandell, J P May, Equivariant orthogonal spectra and S-modules, Mem. Amer. Math. Soc. 755, Amer. Math. Soc. (2002) MR1922205

[19] J P May, Classifying spaces and fibrations, Mem. Amer. Math. Soc. 155, Amer. Math. Soc. (1975) MR0370579

[20] J P May, Enriched model categories and presheaf categories, preprint (2013) arXiv: 1110.3567

[21] C Rezk, A streamlined proof of Goodwillie's n-excisive approximation, Algebr. Geom. Topol. 13 (2013) 1049-1051 MR3044601

[22] E Riehl, Categorical homotopy theory, New Mathematical Monographs 24, Cambridge Univ. Press (2014) MR3221774

[23] S Schwede, Spectra in model categories and applications to the algebraic cotangent complex, J. Pure Appl. Algebra 120 (1997) 77-104 MR1466099

[24] S Schwede, Lecture notes on equivariant stable homotopy theory, unpublished (2013) Available at http://www.math.uni-bonn.de/people/schwede/

[25] B Shipley, A convenient model category for commutative ring spectra, from: "Homotopy theory: relations with algebraic geometry, group cohomology, and algebraic $K$-theory", (P Goerss, S Priddy, editors), Contemp. Math. 346, Amer. Math. Soc. (2004) 473-483 MR2066511

[26] M Stephan, Elmendorf's theorem for cofibrantly generated model categories, Master's thesis, ETH Zurich (2010) Available at http://www.math.ku.dk/ jg/students/ stephan.msthesis.2010.pdf

[27] J Thévenaz, P J Webb, Homotopy equivalence of posets with a group action, J. Combin. Theory Ser. A 56 (1991) 173-181 MR1092846

[28] R W Thomason, Homotopy colimits in the category of small categories, Math. Proc. Cambridge Philos. Soc. 85 (1979) 91-109 MR510404 
[29] R Villarroel-Flores, Equivariant homotopy type of categories and preordered sets, PhD thesis, University of Minnesota (1999) MR2699714 Available at http:// search. proquest. com/docview/304524888

[30] R Villarroel-Flores, The action by natural transformations of a group on a diagram of spaces, preprint (2004) arXiv:math/0411502v1

[31] F Waldhausen, Algebraic K-theory of spaces, from: "Algebraic and geometric topology”, (A Ranicki, N Levitt, F Quinn, editors), Lecture Notes in Math. 1126, Springer, Berlin (1985) 318-419 MR802796

Department of Mathematics, Massachusetts Institute of Technology

77 Massachusetts Avenue, Cambridge, MA 02139-4307, USA

Department of Mathematical Sciences, University of Copenhagen

Universitetsparken 5, DK-2100 Copenhagen, Denmark

dotto@mit.edu, moi@wwu.de

http://math.mit.edu/ dotto/, http://www.math.uni-muenster.de/u/moi/

Received: 2 September $2014 \quad$ Revised: 11 April 2015 
